\title{
PARITY VIOLATION IN FORWARD ANGLE ELASTIC ELECTRON-PROTON SCATTERING
}

\author{
Grady Wilson Miller IV
}

\author{
A DISSERTATION \\ PRESENTED TO THE FACULTY \\ of Princeton University \\ IN CANDIDACY FOR THE DEGREE \\ of Doctor of Philosophy \\ RECOMMENDED FOR ACCEPTANCE \\ BY THE DEPARTMENT OF \\ Physics
}

JANUARY 2001 
Copyright (C) 2000 by Grady Wilson Miller IV. All rights reserved. 


\begin{abstract}
We have measured the parity-violating electroweak asymmetry in the elastic scattering of polarized electrons from the proton at Jefferson Laboratory. The kinematic point $\left(\left\langle\theta_{l a b}\right\rangle=12.3^{\circ}\right.$ and $\left.\left\langle Q^{2}\right\rangle=0.48(\mathrm{GeV} / \mathrm{c})^{2}\right)$ is chosen to provide sensitivity to the strange electric form factor $G_{E}^{s}$.

A $3.36 \mathrm{GeV}$ beam of longitudinally polarized electrons was scattered from protons in a liquid hydrogen target. The scattered flux was detected by a pair of spectrometers which focussed the elastically-scattered electrons onto total-absorption detectors. The detector signals were integrated and digitized by a custom data acquisition system. A feedback system reduced systematic errors by controlling helicity-correlated beam intensity differences at the sub-ppm (part per million) level.

The experimental result, $A=-14.5 \pm 2.0$ (stat) \pm 1.1 (syst) ppm, is consistent with the electroweak Standard Model with no additional contributions from strange quarks. In particular, the measurement implies $G_{E}^{s}+0.39 G_{M}^{s}=0.023 \pm 0.040 \pm$ $0.026\left(\delta G_{E}^{n}\right)$, where the last uncertainty is due to the estimated uncertainty in the neutron electric form factor $G_{E}^{n}$. This result represents the first experimental constraint of the strange electric form factor.
\end{abstract}




\section{Acknowledgements}

I would like to thank my advisor Krishna Kumar for his guidance and for coming through for me when it counted. I would also like to thank Paul Souder for showing me how to think like an experimental physicist and how to get things done. Thanks to Bob Michaels, Mike Finn, Matt Poelker, John Hansknecht, and the rest of Jefferson Lab for making this experiment possible. I would also like to thank Bill Kahl, Gary Rutledge, Peter Mastromarino, Mark Spradlin, and especially Richard Holmes for helping to make it all happen.

I would like to thank Gordon Cates for useful discussions about things other than physics and for looking out for me at the end. My parents Mary and Grady Miller as well as Ed and Maggie Johnson deserve special thanks for their support and encouragement throughout. Thanks also to Jed for being my brother and to Paul and James Johnson for helping my family move every year. I would like to thank my dear children Quinton and Phoebe for serving as my inspiration and reminding me what really matters. Most of all, I would like to thank my wife Helen, without whose love, support, patience, and sacrifice I would not be where I am today. 


\section{Contents}

1 Theory and Formalism 1

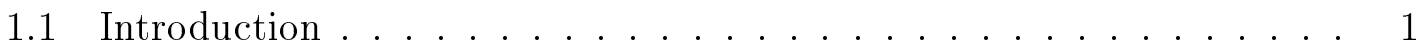

1.2 Electron-Nucleon Scattering . . . . . . . . . . . . 2

1.2.1 Electromagnetic Form Factors . . . . . . . . . . . 3

1.2.2 Neutral Weak Form Factors ............... 6

1.2.3 Relationship Among the Form Factors . . . . . . . . . 7

1.3 Parity Violation . . . . . . . . . . . . . . . 10

2 Experimental Design 13

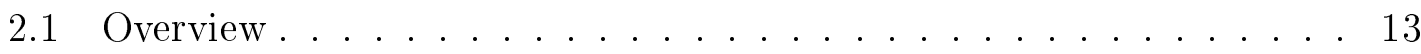

Choice of Kinematics . . . . . . . . . . . . 14

Experimental Overview ................. 15

2.2 Experimental Technique ................... 17

2.2.1 Helicity Reversal Scheme . . . . . . . . . . . . . 18

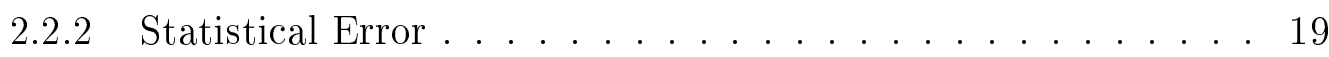

Rate Estimate .................... 20

2.2.3 Systematic Error ................... 21

Detector Linearity ................. 23

2.3 Helicity-Correlated Systematics . . . . . . . . . . . 25

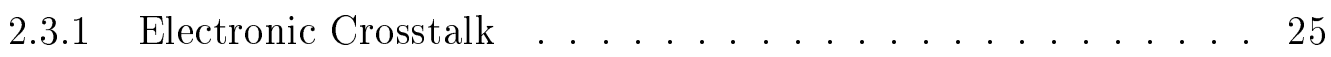


2.3 .2 Intensity Differences $\ldots \ldots \ldots \ldots \ldots$

PITA Effect . . . . . . . . . . . . 28

Feedback System . . . . . . . . . . . . . . . 30

Feedback Implementation . . . . . . . . . . . . 32

Parameters of Feedback Scheme . . . . . . . . . . . . . 34

2.3.3 Position, Angle, and Energy Differences . . . . . . . . . . 35

3 Description of the Apparatus $\quad 39$

3.1 Accelerator . . . . . . . . . . . . . . . 39

3.2 Polarized Source Laser . . . . . . . . . . . . . . . . . . . 40

3.3 Helicity Control Electronics _. . . . . . . . . . . . . . . . 42

3.4 Beam Monitors . . . . . . . . . . . . . . . . . . . 44

3.4.1 Beam Position Monitors . . . . . . . . . . . . . . . 45

3.4.2 Beam Current Monitors . . . . . . . . . . . . . 46

3.5 Target and Raster . . . . . . . . . . . . . . . . . . 47

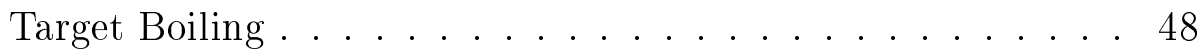

3.6 Spectrometers . . . . . . . . . . . . . . 50

3.7 Detectors $\ldots \ldots \ldots \ldots \ldots \ldots \ldots \ldots \ldots \ldots$

3.8 Data Acquisition System and Integrating Electronics . . . . . . . 54

Integrating ADC Boards . . . . . . . . . . . . 55

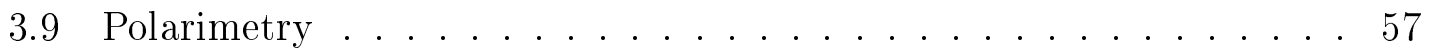

3.9 .1 Mott Polarimeter . . . . . . . . . . . . . . 58

3.9.2 Møller Polarimeter . . . . . . . . . . . . . . . . . . . . . 59

4 Analysis and Results $\quad 62$

$4.1 \quad$ Raw Asymmetries . . . . . . . . . . . . . . . . . 62

4.2 Passive Helicity Reversal . . . . . . . . . . . . . . . . 65 
4.3 Performance of Intensity Feedback System . . . . . . . . . . . 66

4.4 Beam Modulation Analysis . . . . . . . . . . . . . 70

4.5 Auxiliary Data Runs ... . . . . . . . . . . . 73

4.5.1 Determination of $\left\langle Q^{2}\right\rangle \ldots \ldots \ldots . \ldots . \ldots 74$

4.5.2 Background Estimation . . . . . . . . . . . . 74

4.6 Final Experimental Asymmetry . . . . . . . . . . . . . 77

$\begin{array}{lll}5 & \text { Conclusion } & 78\end{array}$

5.1 Extracting Strange Form Factors . . . . . . . . . . . 78

5.2 Theoretical Implications . . . . . . . . . . . . . . . 80

5.3 Future Directions ...................... 82

$\begin{array}{ll}\text { A Electronics Handbook } & 84\end{array}$

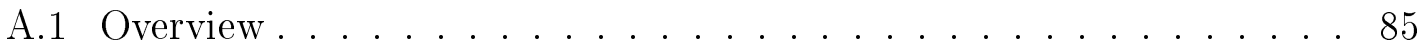

A.2 Helicity Control Electronics . . . . . . . . . . . 86

A.2.1 High Voltage Supplies and Switcher . . . . . . . . . . 87

A.2.2 Helicity Generator . . . . . . . . . . . 88

Instructions for Using the Helicity Generator . . . . . . . . 88

Description of the Output Signals . . . . . . . . . . 90

Pseudorandom Bit Generator . . . . . . . . . . . . 91

Running in Toggle Mode . . . . . . . . . . . . 93

Predicting the Pseudorandom Bit Sequence . . . . . . . . 93

A.2.3 HV Setpoint Generator . . . . . . . . . . . . . 94

Instructions for Using the HV Setpoint Generator . . . . . . 95

Functional Details ................ 95

A.3 Data Acquisition Electronics . . . . . . . . . . . . . 98

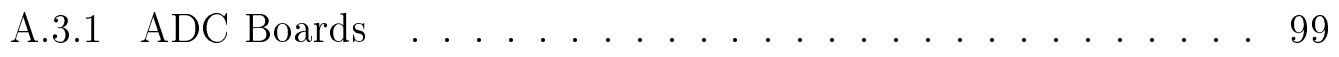


Principles of Operation . . . . . . . . . . . . . 100

ADC Performance . . . . . . . . . . . . . . . 103

Instructions for Using the ADC Boards . . . . . . . . . . 106

A.3.2 ADC Timing Board . . . . . . . . . . . . . . . . . . 114

External Connections and Internal Addresses . . . . . . . 115

ADC Timing . . . . . . . . . . . . . . 115

Input Signals . . . . . . . . . . . . . . . 118

DACs and $V / F \ldots \ldots \ldots \ldots \ldots \ldots$ 


\section{List of Figures}

1.1 Feynman diagrams for electron-proton scattering . . . . . . . . 3

1.2 Nucleon electromagnetic form factors . . . . . . . . . . 6

2.1 Overview of the experimental apparatus. . . . . . . . . . . 14

2.2 Figure of Merit . . . . . . . . . . . . . . 15

2.3 Sample helicity sequence . . . . . . . . . . . . . . 19

2.4 Feedback system. . . . . . . . . . . . . . . 30

2.5 PITA curve. . . . . . . . . . . . . . . . 31

2.6 Diagram of BPMs and steering coils along beamline . . . . . . . . 37

2.7 Beam modulation cycle . . . . . . . . . . . . . 38

3.1 Continuous Electron Beam Accelerator Facility . . . . . . . . . . 40

3.2 The polarized source laser. . . . . . . . . . . . . . 41

3.3 Helicity control electronics. . . . . . . . . . . . . . . 43

3.4 Diagram of beam position monitor. . . . . . . . . . . . . 44

3.5 Beam position monitor resolution ............. 46

3.6 Beam current monitor resolution . . . . . . . . . . . . . 47

3.7 Target boiling test: Plot of detector widths vs. beam current . . . . . 49

3.8 Target boiling test: Plot of extrapolated detector widths . . . . . . 50

3.9 Hall A high-resolution spectrometer. . . . . . . . . . . . . 51

3.10 Scattering events at spectrometer focal plane. . . . . . . . . . . 52 
3.11 HAPPEX detector. ..................... 53

3.12 Data acquisition system. . . . . . . . . . . . 55

3.13 Circuit diagram of ADC channel . . . . . . . . . . . . . . 56

3.14 Diagram of the Mott polarimeter . . . . . . . . . . 59

3.15 Diagram of the Møller polarimeter. . . . . . . . . . . 60

4.1 Run-by-run plot of raw asymmetry for each detector . . . . . . . 63

4.2 Histogram of all raw pair asymmetries . . . . . . . . . . . . 64

4.3 Plot of average raw asymmetry for each $\lambda / 2$ data set . . . . . . 65

4.4 Demonstration of feedback effectiveness. . . . . . . . . . 67

4.5 Run-by-run plot of intensity asymmetry. . . . . . . . . . . . 68

4.6 Demonstration of $1 / N$ convergence of feedback. . . . . . . . . . 69

4.7 Example of feedback divergence. . . . . . . . . . . . . 70

4.8 Plot of beam loading. . . . . . . . . . . . . . 71

4.9 Plot of helicity-correlated position, angle, and energy differences. . . . 73

4.10 Histogram of $Q^{2}$ distribution. . . . . . . . . . . . . . 75

4.11 Sensitivity of the HAPPEX detector to inelastic background. . . . . . 76

5.1 Theoretical calulations of $\left(A_{n s}-A_{\text {exp }}\right) / A_{n s} \ldots \ldots$........ 81

A.1 Electronics overview . . . . . . . . . . . . 85

A.2 Block diagram of helicity control electronics. . . . . . . . . 86

A.3 Circuit diagram of Helicity Generator. . . . . . . . . . . . 89

A.4 Sample timing diagram for Helicity Generator . . . . . . . . . . . 90

A.5 Pseudorandom bit generator. . . . . . . . . . . . . 92

A.6 Circuit diagram of HV Setpoint Generator. . . . . . . . . . . 97

A.7 Data acquisition electronics. . . . . . . . . . . . . 99 
A.8 Circuit diagram of ADC channel. . . . . . . . . . . . . 100

A.9 Timing diagram for ADC integration cycle. . . . . . . . . . . . 102

A.10 ADC pedestal noise. . . . . . . . . . . . . . . . . . . . 104

A.11 Integral nonlinearity of ADC channel. . . . . . . . . . . . . . . 105

A.12 ADC front panel connections. . . . . . . . . . . . . . 107

A.13 ADC response to noise DAC. . . . . . . . . . . . 111

A.14 Voltage-intgrating ADC channel. . . . . . . . . . . . . . . 112

A.15 Current-integrating ADC channel. . . . . . . . . . . . . . . . 114

A.16 Front panel of timing card. . . . . . . . . . . . . . 116

A.17 Timing diagram for ADC Timing Board outputs. . . . . . . . 118

A.18 Circuit diagram of ADC Timing Board. . . . . . . . . . 120 


\section{List of Tables}

2.1 Battery asymmetries. . . . . . . . . . . . . 26

4.1 Average raw asymmetries . . . . . . . . . . . . 66

4.2 Sensitivities of normalized detector flux to beam parameters. . . . . . 72

4.3 Summary of errors for $A_{\text {raw }}$ and $A_{\text {exp }} \ldots \ldots \ldots \ldots$. . . . . 77

5.1 Various theoretical predictions for $\rho_{s}$ and $\mu_{s} \ldots \ldots . \ldots 2$

A.1 Address map for ADC board . . . . . . . . . . . . . 109

A.2 Address map for ADC Timing Board. . . . . . . . . . . . . 117 


\section{Chapter 1}

\section{Theory and Formalism}

\subsection{Introduction}

Ever since Frisch and Stern discovered the proton's anomalous magnetic moment in 1933, first hinting that the proton is a composite particle, scientists have struggled to understand the proton's internal structure. Deep inelastic scattering experiments in the late 1960s verified that the proton contains hard, point-like quarks, but subsequent experiments have shown that simple quark models are too naive. These experimental results, along with the emergence of QCD in the 1970s, have painted a picture of the proton as quite a complex object.

The proton is believed to be a relativistic bound state of three valence quarks (two up quarks and one down quark) held together by gluons amidst an evanescent sea of quark-antiquark pairs. Since the mass of the strange quark is comparable to the proton mass and to the scale of the strong interaction, it is possible that strange quarks could be a significant component of this sea. Indeed, recent theo-

retical investigations $[1,2]$ based on experimental measurements of deep-inelastic polarized lepton-nucleon scattering and measurements of the pion-nucleon $\Sigma$ term suggest that strange quark scalar and axial vector matrix elements are sizable and may contribute to nucleon properties at the level of 10-15\%. In light of these re- 
sults, it is reasonable to ask whether strange quark vector matrix elements might also contribute significantly to nucleon properties such as its charge and magnetic moment distributions [3]. Since little is known about nucleon structure from first principles, the question must be addressed experimentally.

A particularly clean experimental technique $[4,5,6]$ for isolating the effects of strange quarks in the nucleon is the measurement of parity-violating amplitudes arising from electroweak interference in the elastic scattering of polarized electrons from the proton [7]. While electromagnetic electron-proton scattering reveals the charge and magnetic moment distributions of the proton, resolving the flavor structure of these properties requires another degree of freedom. The neutral weak force provides this additional degree of freedom and can be used to probe for strange quark effects.

This thesis presents an experiment which measures the parity-violating asymmetry in the elastic scattering of polarized electrons from the proton. The remainder of this chapter will show how strange quark effects can be extracted from a measurement of this asymmetry.

\subsection{Electron-Nucleon Scattering}

In order to show how a measurement of the parity-violating asymmetry in elastic electron-proton scattering can reveal information about strange quarks in the proton, we first must develop the language and formalism of elastic electron-nucleon scattering. 
(a)

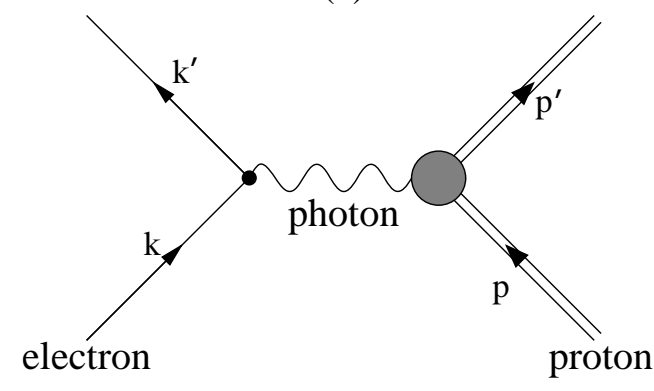

(b)

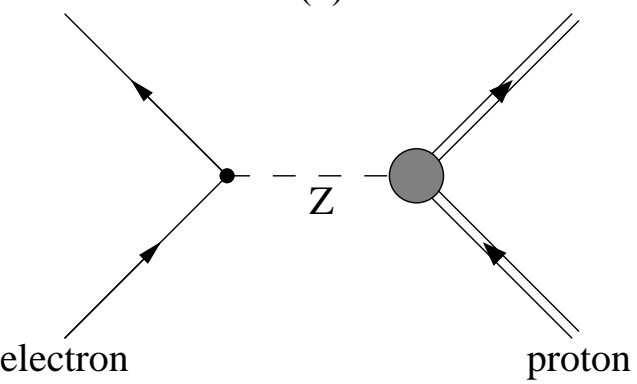

Figure 1.1: Feynman diagrams for electron-proton scattering. Electromagnetic scattering is depicted in (a). Neutral weak scattering is depicted in (b).

\subsubsection{Electromagnetic Form Factors}

Consider the elastic electromagnetic scattering of electrons from nucleons, represented in lowest order by the Feynman diagram in Figure 1.1. The amplitude for this process can be written

$$
\mathcal{M}=\frac{1}{q^{2}} j^{\mu} J_{\mu}
$$

where

$$
j^{\mu}=-e \bar{u}\left(k^{\prime}\right) \gamma^{\mu} u(k)
$$

is the electron current. The nucleon current $J_{\mu}$ would be written similarly if the nucleon were an elementary particle. Instead we write $J_{\mu}$ in the most general form permitted by Lorentz covariance together with parity and time-reversal invariance [2]:

$$
J_{\mu}=e \bar{u}_{N}\left(p^{\prime}\right)\left[\gamma_{\mu} F_{1}\left(q^{2}\right)+\frac{i \sigma_{\mu \nu} q^{\nu}}{2 m_{N}} F_{2}\left(q^{2}\right)\right] u_{N}(p)
$$

where the form factors $F_{1}$ and $F_{2}$ have been introduced. These form factors can only depend on $q^{2}$, since this is the only independent scalar variable at the vertex.

The form factors $F_{1}$ and $F_{2}$ are called the Dirac and Pauli form factors, respectively. In practice, it is more common to use linear combinations of $F_{1}$ and $F_{2}$, 
known as the Sachs form factors

$$
G_{E} \equiv F_{1}-\tau F_{2} \text { and } G_{M} \equiv F_{1}+F_{2},
$$

where $\tau \equiv-q^{2} / 4 m_{N}^{2}$. The Sachs form factors $G_{E}$ and $G_{M}$ are referred to as the electric and magnetic form factors, respectively. This is because for $|\mathbf{q}|^{2} \ll m_{N}^{2}$, that is, when the nucleon does not recoil significantly, the form factors $G_{E}\left(q^{2}\right)$ and $G_{M}\left(q^{2}\right)$ are the Fourier transforms of the nucleon's electric charge and magnetic moment distributions, respectively. In the limit as $q^{2} \rightarrow 0$, the electric form factor is simply the net charge and the magnetic form factor is the net magnetic moment of the nucleon:

$$
G_{E}\left(q^{2}=0\right)=\frac{Q}{e}, \quad G_{M}\left(q^{2}=0\right)=\frac{\mu}{e / 2 m_{N}} .
$$

Specifically, therefore, $G_{E}(0)$ and $G_{M}(0)$ for the proton and the neutron are

$$
\begin{array}{ll}
G_{E}^{p}(0)=1, & G_{M}^{p}(0)=\mu_{p}=2.79 \\
G_{E}^{n}(0)=0, & G_{M}^{n}(0)=\mu_{n}=-1.91 .
\end{array}
$$

The mean square charge radius $\left\langle r^{2}\right\rangle$ of the proton is related to the slope of the electric form factor at $q^{2}=0$ by

$$
\left\langle r^{2}\right\rangle=6\left(\frac{d G_{E}^{p}\left(q^{2}\right)}{d q^{2}}\right)_{q^{2}=0},
$$

as suggested by the expansion of the form factor in orders of $q^{2}$. The evolution of the form factors for $-q^{2}>0$ must be determined experimentally.

Equation 1.1 can be used to calculate the differential cross section for elastic electron-proton scattering. The result is known as the Rosenbluth formula [8]:

$$
\left.\frac{d \sigma}{d \Omega}\right|_{l a b}=\frac{\alpha^{2}}{4 E^{2} \sin ^{4}(\theta / 2)} \frac{E^{\prime}}{E}\left(\frac{\left(G_{E}^{p}\right)^{2}+\tau\left(G_{M}^{p}\right)^{2}}{1+\tau} \cos ^{2} \frac{\theta}{2}+2 \tau\left(G_{M}^{p}\right)^{2} \sin ^{2} \frac{\theta}{2}\right) .
$$


The proton form factors $G_{E}^{p}$ and $G_{M}^{p}$ have been determined experimentally using this formula by scattering electrons from the protons in a hydrogen target and measuring $d \sigma / d \Omega$ as a function of $q^{2}$ and lab scattering angle $\theta$. It is found that $G_{E}^{p}$ and $G_{M}^{p}$ appear to have the same functional form up to a scale factor. The empirical data is fit surprisingly well by a dipole function $G_{D}$ given by

$$
G_{D}\left(q^{2}\right)=\left(1-\frac{q^{2}}{0.71}\right)^{-2}=(1+4.97 \tau)^{-2}
$$

with the proton form factors given by

$$
G_{E}^{p}=G_{D} \text { and } G_{M}^{p}=\mu_{p} G_{D} .
$$

The neutron form factors $G_{E}^{n}$ and $G_{M}^{n}$ are more difficult to measure, since there is no free neutron target. They are typically measured either by scattering electrons from deuterons and subtracting out the theoretical proton contribution or by scattering neutrons from atomic electrons. Consequently the neutron form factors are known less precisely. It is found that the neutron magnetic form factor has the same dipole dependence as the proton form factors:

$$
G_{M}^{n}=\mu_{n} G_{D}
$$

The neutron electric form factor cannot have the same dipole form as the other three form factors, however, since it is constrained by $G_{E}^{n}(0)=0$. It is found that $G_{E}^{n}$ can be approximated by the function

$$
G_{E}^{n}=\frac{-\mu_{n} \tau G_{D}}{1+5.6 \tau}
$$

Equation 1.9 is known as the Galster parameterization for $G_{E}^{n}[9]$.

The dipole and Galster parameterizations of the proton and neutron electromagnetic form factors are plotted as functions of $-q^{2}$ in Figure 1.2. 


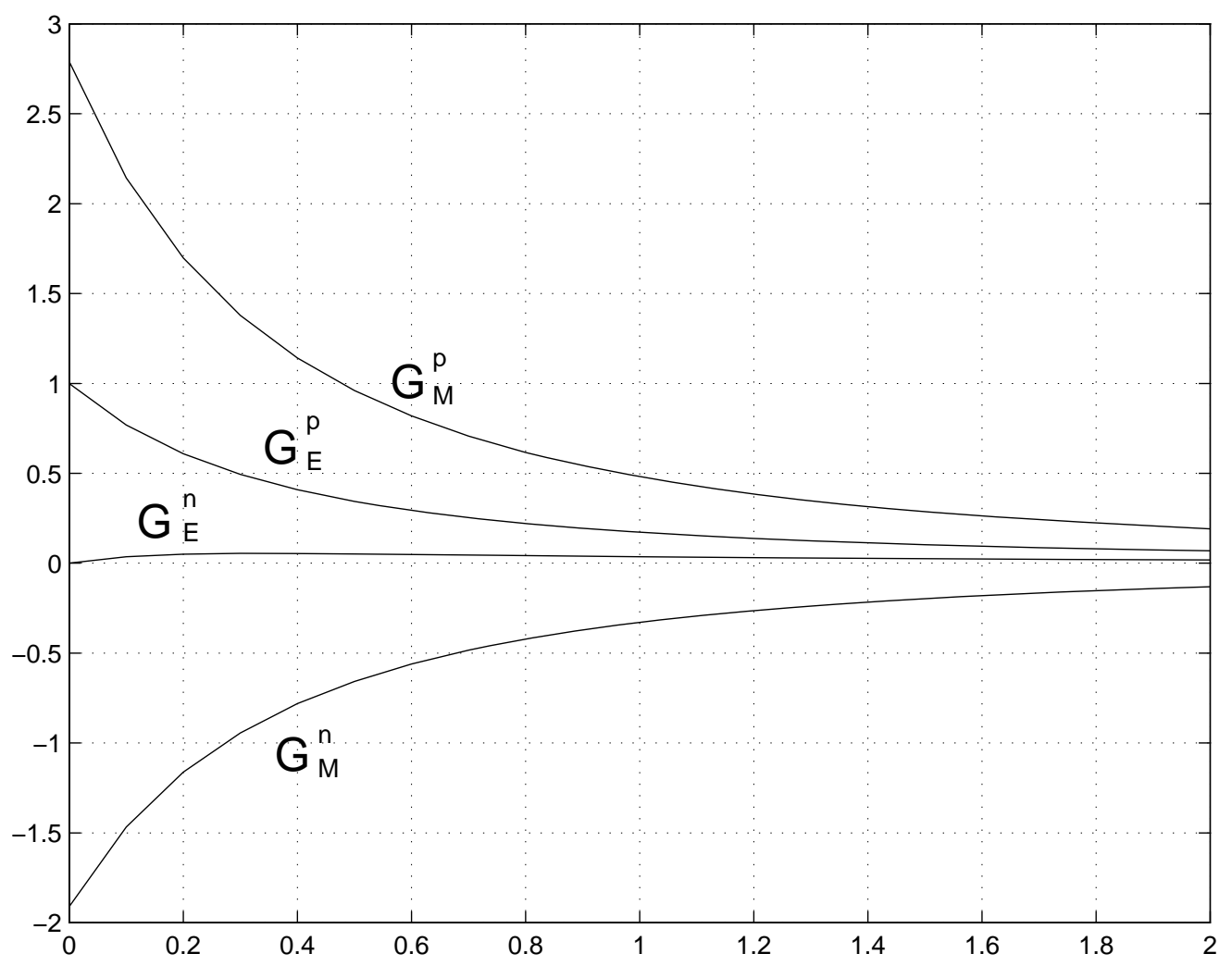

Figure 1.2: A plot of the dipole and Galster parameterizations of the nucleon electromagnetic form factors for $0<-q^{2}<2$.

\subsubsection{Neutral Weak Form Factors}

Electron-proton scattering may also proceed through exchange of a virtual $Z^{0}$, represented in lowest order by the Feynman diagram in Figure 1.1. This process gives rise to a new current for the proton. We label this neutral weak current $J_{\mu}^{Z}$ to distinguish it from the electromagnetic current in Section 1.2.1 which we now label $J_{\mu}^{\gamma}$

The amplitude for neutral weak electron-proton scattering is given by

$$
\mathcal{M}=\frac{G_{F}}{\sqrt{2}} j^{Z \mu} J_{\mu}^{Z}
$$


where the electron current is written

$$
j^{Z \mu}=\bar{u}_{e} \gamma^{\mu}\left(c_{V}^{e}-c_{A}^{e} \gamma^{5}\right) u_{e}
$$

and, again, we write the proton current in the most general form possible:

$$
J_{\mu}^{Z}=\bar{u}_{p}\left[\gamma_{\mu} F_{1}^{Z}\left(q^{2}\right)+\frac{i \sigma_{\mu \nu} q^{\nu}}{2 M_{p}} F_{2}^{Z}\left(q^{2}\right)+\gamma_{\mu} \gamma_{5} G_{A}^{Z}\right] u_{p} .
$$

Here we have introduced three new form factors for the proton, each of which depends only on $q^{2}$. The weak neutral form factors $F_{1}^{Z}$ and $F_{2}^{Z}$ are analogous to the electromagnetic form factors $F_{1}^{\gamma}$ and $F_{2}^{\gamma}$, and in fact they are intimately related, as will be discussed in the next section.

\subsubsection{Relationship Among the Form Factors}

The relationship among the electromagnetic and neutral weak form factors may be established by examining the flavor structure of the nucleon currents. In electronnucleon scattering, the gauge bosons are actually coupling to quarks in the nucleon. Thus the nucleon vector currents $J_{\mu}^{\gamma}$ and $J_{\mu}^{Z}$ may be decomposed into a sum of quark currents, one current for each quark flavor.

The electromagnetic and neutral weak nucleon vector currents $J_{\mu}^{\gamma}$ and $J_{\mu}^{Z}$ may be written as nucleonic matrix elements of the quark current operators $\hat{J}_{\mu}^{\gamma}$ and $\hat{J}_{\mu}^{Z}$,

$$
J_{\mu}^{\gamma} \equiv\left\langle N\left|\hat{J}_{\mu}^{\gamma}\right| N\right\rangle, \quad J_{\mu}^{Z} \equiv\left\langle N\left|\hat{J}_{\mu}^{Z}\right| N\right\rangle
$$

where $|N\rangle$ is a proton or a neutron and the quark current operators are given by

$$
\hat{J}_{\mu}^{\gamma} \equiv \sum_{q} Q_{q} \bar{q} \gamma_{\mu} q, \quad \hat{J}_{\mu}^{Z} \equiv \sum_{q} g_{q}^{V} \bar{q} \gamma_{\mu} q
$$

Here $Q_{q}$ and $g_{q}^{V}$ are the Standard Model electromagnetic and neutral weak vector quark couplings, respectively, and the sums are over all quark flavors $q$. Since it 
is assumed that only $u, d, s$ quarks contribute significantly to nucleonic properties, the sums in Equation 1.10 may be limited to these quark flavors and the nucleon currents can be written explicitly in terms of the quark-nucleon currents as:

$$
\begin{aligned}
J_{\mu}^{\gamma}=\left\langle N\left|\hat{J}_{\mu}^{\gamma}\right| N\right\rangle= & \frac{2}{3}\left\langle N\left|\bar{u} \gamma_{\mu} u\right| N\right\rangle-\frac{1}{3}\left\langle N\left|\bar{d} \gamma_{\mu} d\right| N\right\rangle-\frac{1}{3}\left\langle N\left|\bar{s} \gamma_{\mu} s\right| N\right\rangle, \\
J_{\mu}^{Z}=\left\langle N\left|\hat{J}_{\mu}^{Z}\right| N\right\rangle= & \left(\frac{1}{4}-\frac{2}{3} \sin ^{2} \theta_{W}\right)\left\langle N\left|\bar{u} \gamma_{\mu} u\right| N\right\rangle \\
& +\left(-\frac{1}{4}+\frac{1}{3} \sin ^{2} \theta_{W}\right)\left\langle N\left|\bar{d} \gamma_{\mu} d\right| N\right\rangle \\
& +\left(-\frac{1}{4}+\frac{1}{3} \sin ^{2} \theta_{W}\right)\left\langle N\left|\bar{s} \gamma_{\mu} s\right| N\right\rangle .
\end{aligned}
$$

Notice that the same quark-nucleon currents, $\left\langle N\left|\bar{u} \gamma_{\mu} u\right| N\right\rangle,\left\langle N\left|\bar{d} \gamma_{\mu} d\right| N\right\rangle$, and $\left\langle N\left|\bar{s} \gamma_{\mu} s\right| N\right\rangle$, appear on the right-hand side of both Equations 1.11 and 1.12. The quark-nucleon currents depend only on the vector nature of the coupling, not on whether the coupling is through a photon or a $Z^{0}$. This generality will allow us to relate the electromagnetic form factors to the neutral weak and strange quark form factors.

In Sections 1.2.1 and 1.2.2, the nucleon currents were written in the most general form possible by introducing electromagnetic and neutral weak form factors $F_{1,2}^{\gamma}$ and $F_{1,2}^{Z}$. Each of these form factors is associated with a particular nucleon, so there are actually eight nucleonic vector form factors which may be listed compactly as $F_{(1,2)}^{(p, n)(\gamma, Z)}$. The quark-nucleon currents may similarly be written in the most general form possible by introducing quark-nucleon form factors $F_{(1,2)}^{(p, n)(u, d, s)}$ defined by

$$
\left\langle N\left|\bar{q} \gamma_{\mu} q\right| N\right\rangle \equiv \bar{u}_{N}\left[\gamma_{\mu} F_{1}^{N q}+\frac{i \sigma_{\mu \nu} q^{\nu}}{2 m_{N}} F_{2}^{N q}\right] u_{N}
$$

where $q=u, d$, or $s$ and $N=p$ or $n$.

Rewriting Equation 1.11 in terms of form factors,

$$
J_{\mu}^{\gamma}=Q \bar{u}_{N}\left[\gamma_{\mu} F_{1}^{N \gamma}+\frac{i \sigma_{\mu \nu} q^{\nu}}{2 m_{N}} F_{2}^{N \gamma}\right] u_{N}=\sum_{q=u, d, s} Q_{q} \bar{u}_{N}\left[\gamma_{\mu} F_{1}^{N q}+\frac{i \sigma_{\mu \nu} q^{\nu}}{2 m_{N}} F_{2}^{N q}\right] u_{N}
$$


we see that the ordinary nucleon electromagnetic form factors may be expressed as sums of the quark-nucleon form factors with coefficients given by the Standard Model quark couplings:

$$
F_{1,2}^{N \gamma}=\sum_{q=u, d, s} Q_{q} F_{1,2}^{N q}=\frac{2}{3} F_{1,2}^{N u}-\frac{1}{3} F_{1,2}^{N d}-\frac{1}{3} F_{1,2}^{N s} .
$$

The nucleon neutral weak vector form factors may be expressed similarly:

$$
F_{1,2}^{N Z}=\sum_{q=u, d, s} g_{q}^{V} F_{1,2}^{N q} .
$$

Identical equations hold for the Sachs form factors, defined in Equation 1.4. In particular, $G_{E, M}^{p \gamma}, G_{E, M}^{n \gamma}$, and $G_{E, M}^{p Z}$ may be written

$$
\begin{gathered}
G_{E, M}^{p \gamma}=\frac{2}{3} G_{E, M}^{p u}-\frac{1}{3} G_{E, M}^{p d}-\frac{1}{3} G_{E, M}^{p s}, \\
G_{E, M}^{n \gamma}=\frac{2}{3} G_{E, M}^{n u}-\frac{1}{3} G_{E, M}^{n d}-\frac{1}{3} G_{E, M}^{n s}, \\
G_{E, M}^{p Z}=\left(\frac{1}{4}-\frac{2}{3} \sin ^{2} \theta_{W}\right) G_{E, M}^{p u}+\left(-\frac{1}{4}+\frac{1}{3} \sin ^{2} \theta_{W}\right) G_{E, M}^{p d}+\left(-\frac{1}{4}+\frac{1}{3} \sin ^{2} \theta_{W}\right) G_{E, M}^{p s} .
\end{gathered}
$$

These three equations may be condensed by invoking strong isospin symmetry. Under the assumption that the up (down) quarks in the proton have the same properties as the down (up) quarks in the neutron, we have $G^{p u}=G^{\text {nd }}$ and $G^{p d}=$ $G^{n u}$. Also, $G^{n s}=G^{p s} \equiv G^{s}$, since strange quarks appear due to strong processes. Given these identities we can eliminate the up and down quark form factors in favor of the proton and neutron electromagnetic form factors to obtain

$$
G_{E, M}^{p Z}=\left(\frac{1}{4}-\sin ^{2} \theta_{W}\right) G_{E, M}^{p \gamma}-\frac{1}{4} G_{E, M}^{n \gamma}-\frac{1}{4} G_{E, M}^{s} .
$$

This is the desired relationship among the electromagnetic and neutral weak form factors. Since the electromagnetic form factors $G_{E, M}^{p \gamma}$ and $G_{E, M}^{n \gamma}$ are known from previous experiments, the strange quark form factors $G_{E, M}^{s}$ may be determined by measuring the proton's neutral weak form factors $G_{E, M}^{p Z}$. 


\subsection{Parity Violation}

The proton's neutral weak form factors cannot be measured directly through electronproton scattering because electromagnetic scattering dominates over neutral weak scattering. Instead neutral weak amplitudes may be accessed by measuring parityviolation amplitudes arising from interference between the electromagnetic and neutral weak interactions in the elastic scattering of polarized electrons from protons.

Electron-proton scattering can proceed through exchange of either a virtual photon or $Z^{0}$, and there is no way to determine which process is responsible for a given scattering event. The two possible processes, shown in Figure 1.1, have amplitudes $\mathcal{M}^{\gamma}$ and $\mathcal{M}^{Z}$. In order to compute the total cross section for electronproton scattering we must add the two amplitudes before squaring:

$$
\sigma_{\text {tot }} \propto\left|\mathcal{M}^{\gamma}+\mathcal{M}^{Z}\right|^{2}
$$

Because the weak force violates parity, the neutral weak amplitude $\mathcal{M}^{Z}$ depends on the helicity of the incident electron. Thus the proton has different cross sections for right- and left-handed electrons. The difference is usually expressed as an asymmetry $A_{L R}$ defined by

$$
A_{L R} \equiv \frac{\sigma_{R}-\sigma_{L}}{\sigma_{R}+\sigma_{L}}
$$

where $\sigma_{R} \propto\left|\mathcal{M}^{\gamma}+\mathcal{M}_{R}^{Z}\right|^{2}$ is the cross section for electrons of positive helicity and $\sigma_{L} \propto\left|\mathcal{M}^{\gamma}+\mathcal{M}_{L}^{Z}\right|^{2}$ is the cross section for electrons of negative helicity.

In terms of the scattering amplitudes, the asymmetry for electron-proton scattering becomes

$$
\begin{aligned}
A_{L R} & =\frac{\left|\mathcal{M}^{\gamma}+\mathcal{M}_{R}^{Z}\right|^{2}-\left|\mathcal{M}^{\gamma}+\mathcal{M}_{L}^{Z}\right|^{2}}{\left|\mathcal{M}^{\gamma}+\mathcal{M}_{R}^{Z}\right|^{2}+\left|\mathcal{M}^{\gamma}+\mathcal{M}_{L}^{Z}\right|^{2}} \\
& \approx \frac{\left|\mathcal{M}_{R}^{Z}\right|-\left|\mathcal{M}_{L}^{Z}\right|}{\left|\mathcal{M}^{\gamma}\right|}
\end{aligned}
$$


where we have used the fact that $\left|\mathcal{M}^{Z}\right| \ll\left|\mathcal{M}^{\gamma}\right|$. We can estimate the size of the asymmetry by using the approximation

$$
A_{L R} \approx \frac{\left|\mathcal{M}^{Z}\right|}{\left|\mathcal{M}^{\gamma}\right|} \approx \frac{G_{F}}{e^{2} / q^{2}}
$$

which is on the order of $10^{-5}$ for $-q^{2} \sim 0.5 \mathrm{GeV} / \mathrm{c}^{2}$.

The exact scattering amplitudes $\mathcal{M}^{\gamma}$ and $\mathcal{M}_{L, R}^{Z}$ depend on the proton's electromagnetic and neutral weak form factors. A standard calculation [2] yields an expression for the asymmetry in terms of the form factors:

$$
A_{L R}=-\frac{G_{F} Q^{2}}{\pi \alpha \sqrt{2}}\left[\frac{\epsilon G_{E}^{p \gamma} G_{E}^{p Z}+\tau G_{M}^{p \gamma} G_{M}^{p Z}-\frac{1}{2}\left(1-4 \sin ^{2} \theta_{W}\right) \epsilon^{\prime} G_{M}^{p \gamma} G_{A}^{p Z}}{\epsilon\left(G_{E}^{p \gamma}\right)^{2}+\tau\left(G_{M}^{p \gamma}\right)^{2}}\right],
$$

where $G_{E, M}^{p \gamma}$ are the electric and magnetic Sachs form factors for photon exchange, $G_{E, M}^{p Z}$ and $G_{A}^{p Z}$ are the vector and axial vector form factors for $Z^{0}$ exchange, $Q^{2} \equiv$ $-q^{2}>0$ is the four-momentum transfer, and $\epsilon, \tau$, and $\epsilon^{\prime}$ are kinematic quantities defined as

$$
\epsilon \equiv \frac{1}{1+2\left|\mathbf{q}^{2} / Q^{2}\right| \tan ^{2}(\theta / 2)}, \quad \tau \equiv \frac{Q^{2}}{4 m_{p}^{2}}, \quad \text { and } \quad \epsilon^{\prime} \equiv \sqrt{\tau(1+\tau)\left(1-\epsilon^{2}\right)} .
$$

Since the electromagnetic form factors $G_{E, M}^{p \gamma}$ are known from previous experiments, linear combinations of the neutral weak form factors may be determined by measuring the parity-violating asymmetry. Individual form factors may be isolated through prudent choice of kinematics. Since $1-4 \sin ^{2} \theta_{W} \approx 0.07 \ll 1$, the term involving $G_{A}^{p Z}$ makes a relatively small contribution to the asymmetry and in most cases may be neglected. At fixed $Q^{2}$, the contribution of the other terms varies with scattering angle $\theta$. The coefficient in front of $G_{E}^{p Z}$ is maximum at $\theta=0$ and vanishes as $\theta \rightarrow 180^{\circ}$, while the coefficient in front of $G_{M}^{p Z}$ is nonzero at all angles but is larger at larger angles. Hence forward scattering is most sensitive to the electric form factor $G_{E}^{p Z}$, while backward scattering is most sensitive to the magnetic form factor $G_{M}^{p Z}$. 
The SAMPLE collaboration at the MIT-Bates Linear Accelerator Center has measured $G_{M}^{p Z}$ at $Q^{2}=0.1(\mathrm{GeV} / \mathrm{c})^{2}$ by measuring the parity-violating asymmetry at large scattering angles $[10,11]$. We propose to focus on $G_{E}^{p Z}$ by measuring the asymmetry at forward angles. We can then use Equation 1.13 along with known values of $G_{E, M}^{p \gamma}$ and $G_{E, M}^{n \gamma}$ to extract the strange quark form factor $G_{E}^{s}$. We thereby hope to help establish whether strange quarks make a significant contribution to the charge distribution of the proton. 


\section{Chapter 2}

\section{Experimental Design}

Our experimental goal is to measure the parity-violating asymmetry in the elastic scattering of polarized electrons from unpolarized protons in the forward direction. The expected asymmetry is on the order of 10 parts per million (ppm). The small size of the asymmetry drives much of the experimental design. This chapter describes how the experiment was done and why it was done that way, with an emphasis on the techniques used to control systematic errors at the sub-ppm level.

\section{$2.1 \quad$ Overview}

Our experiment is known as the Hall A Proton Parity Experiment, or HAPPEX. The experiment was performed in Experimental Hall A of the Continuous Electron Beam Accelerator Facility (CEBAF) at Jefferson Laboratory in Newport News, Virginia. At the time of the experiment, the accelerator was capable of delivering a $100 \mu \mathrm{A}$ continuous-wave beam of $40 \%$ polarized electrons at energies up to $4 \mathrm{GeV}$. The beam has very stable characteristics, which is desirable for minimizing systematic errors in an asymmetry measurement. Hall A features a liquid hydrogen target and a pair of high-resolution spectrometers for detecting the scattered particles. An overview of the experimental apparatus is shown in Figure 2.1. 


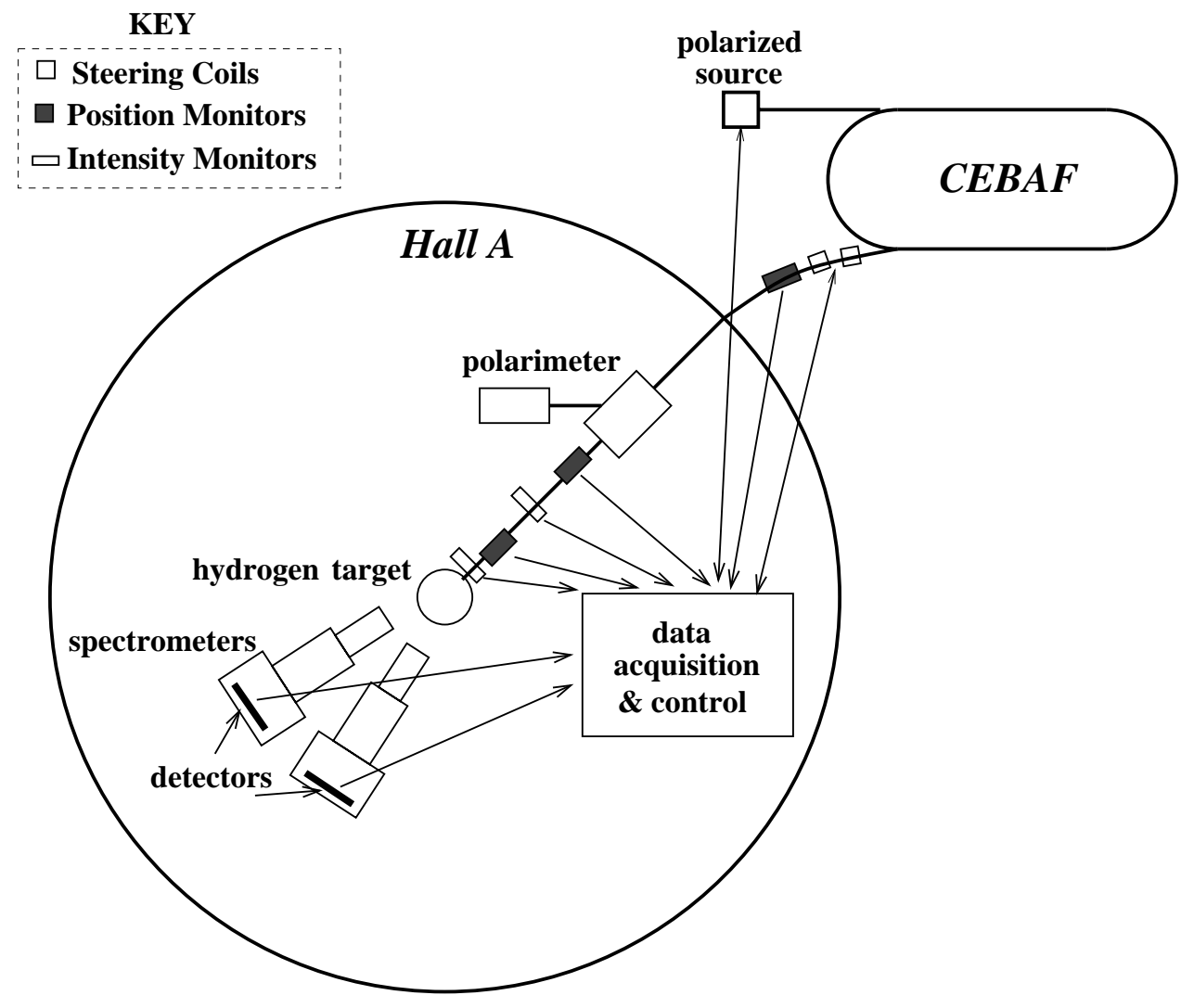

Figure 2.1: Overview of the experimental apparatus.

\section{Choice of Kinematics}

The kinematic point was chosen based on the Figure of Merit in combination with the capabilities of Jefferson Laboratory. In order to maximize sensitivity to $G_{E}^{s}$, as discussed in Section 1.3, the Hall A spectrometers were placed at the most forward scattering angle possible, $\theta_{l a b}=12.3^{\circ}$. The quantity $A^{2} d \sigma / d \Omega$, where $A$ is the asymmetry and $d \sigma / d \Omega$ is the differential cross section at a given kinematic point, is known as the Figure of Merit for an asymmetry measurement. It is a measure of the statistical significance achievable in a given amount of running time.

We would like to maximize the Figure of Merit in order to minimize the fractional statistical error $\Delta A / A$ of the measured asymmetry. The Figure of Merit for our 


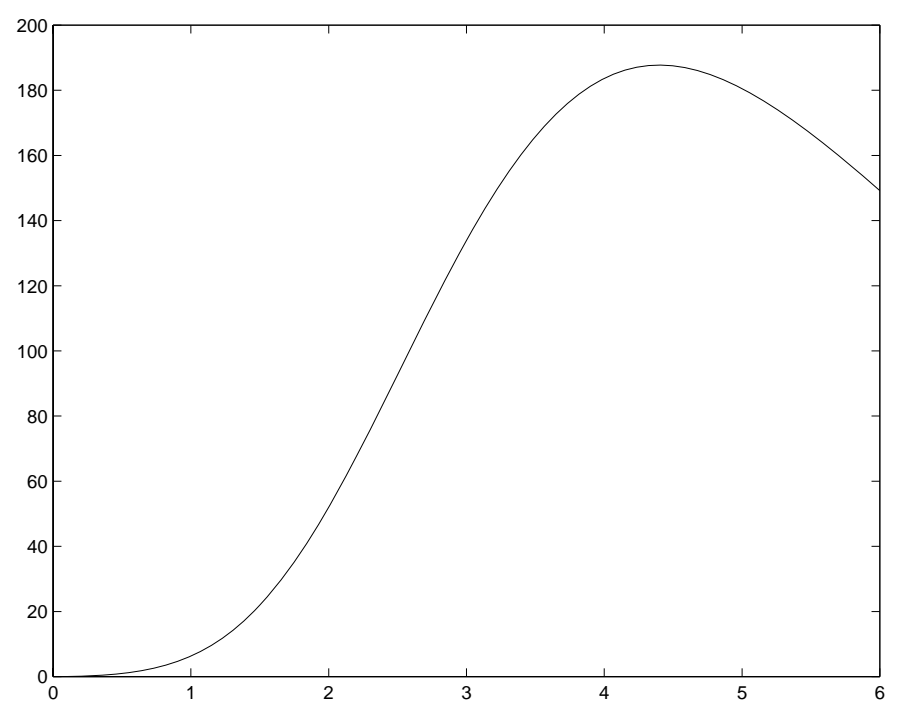

Figure 2.2: The Figure of Merit $A^{2} d \sigma / d \Omega$ for elastic electron-proton scattering is plotted as a function of incident beam energy at a scattering angle of $12.3^{\circ}$. The asymmetry $A$ is calculated from Equation 1.14, assuming $G_{E, M}^{s}=0$ and using parameterizations of the other form factors. The differential scattering cross section $d \sigma / d \Omega$ is calculated using the Rosenbluth formula, given in Equation 1.6.

experiment is shown as a function of beam energy in Figure 2.2. The plot shows that the Figure of Merit peaks for incident beam energies around $4 \mathrm{GeV}$. For our experiment the beam energy was set to $3.356 \mathrm{GeV}$, near the optimum value.

\section{Experimental Overview}

A $3.356 \mathrm{GeV}$ beam of longitudinally-polarized electrons was scattered from protons in a liquid hydrogen target. Two high-resolution spectrometers, each with an angular acceptance of 5.5 millisteradians, were placed symmetrically at $\pm 12.3^{\circ}$ lab scattering angle in order to detect the scattered electrons. Each spectrometer focussed the elastically-scattered electrons onto a total-absorption detector. Use of these highresolution spectrometers, which cleanly separate the elastic peak from the inelastic threshold, allowed us to integrate the detector signal instead of having to count 
individual electrons.

The polarized electrons supplied to the accelerator originated in a bulk GaAs photocathode excited by circularly polarized laser light. The laser light was made circularly polarized using a Pockels cell, and the helicity of the electron beam could be reversed by reversing the handedness of the circular polarization.

The beam helicity was reversed at $30 \mathrm{~Hz}$. Each $33.3 \mathrm{~ms}$ period of constant helicity is referred to as a helicity window. The signals from all detectors and beam monitors were integrated for $32 \mathrm{~ms}$ beginning $700 \mu$ s after the start of each helicity window and the integrated analog result was converted to a digital value. Integration and digitization were performed by custom-built analog-to-digital converter (ADC) boards designed to minimize noise and crosstalk.

In the absence of strange quarks, the expected physics asymmetry from Equation 1.14 is about $15 \mathrm{ppm}$ at this kinematic point. Since the electron beam is only $40 \%$ polarized, we expect to measure a raw asymmetry of about $6 \mathrm{ppm}$. We would like for the total error of the raw asymmetry measurement to be limited by counting statistics of the scattering rate. Thus we attempt to keep measurement noise from broadening the statistical width. We also attempt to keep systematic errors negligible compared with the statistical error. In order to make a $15 \%$ measurement of the raw asymmetry, or $\sim 1$ ppm statistical error, we aim to keep systematic errors in the measurement of the raw asymmetry below $0.1 \mathrm{ppm}$. Meeting these goals ensures that we will achieve the smallest possible experimental error for a given amount of running time.

In the following sections we describe the experimental technique used to cleanly measure small asymmetries and then describe the particular methods we used to deal with helicity-correlated systematic errors. 


\subsection{Experimental Technique}

The asymmetry we will measure is defined as

$$
A_{L R} \equiv \frac{\sigma_{R}-\sigma_{L}}{\sigma_{R}+\sigma_{L}}
$$

where $\sigma_{R}\left(\sigma_{L}\right)$ is the scattering cross section for incident electrons of positive (negative) helicity. To determine the cross-section asymmetry, we only need to measure the scattering cross section for each helicity up to a common scale factor which cancels when the asymmetry is computed. The experimentally measured quantity is the scattered flux normalized to the beam current, henceforth referred to as the normalized flux, which is proportional to the cross section. In order to avoid introducing new notation, we denote the normalized flux by $\sigma$.

The experiment involves two different measurements of the normalized flux, one for each helicity state of the electron beam. We want all experimental parameters except beam helicity to be exactly the same for both measurements. Variations in other experimental parameters, such as beam position and energy, target density, and spectrometer magnetic fields, lead to an increase in the statistical uncertainty of the measured asymmetry if the variations are not correlated with the beam helicity, and can cause a systematic error in the measured asymmetry if the parameter differences are helicity-correlated.

In order to avoid increasing the statistical uncertainty of the measurement due to variations in experimental parameters, we reverse the helicity rapidly, so that the experimental parameters do not have a chance to change much between measurements of the normalized flux. We make a measurement for one helicity, reverse the beam helicity, and immediately make another measurement. We calculate the asymmetry $A_{L R}$ between these measurements of $\sigma_{R}$ and $\sigma_{L}$ using Equation 2.1, repeat millions of times, and average all such asymmetries to obtain the final asymmetry 
with a statistical error given by the standard error in the mean.

In this way we essentially keep track of the difference between $\sigma_{R}$ and $\sigma_{L}$, which is the quantity we care about, rather than their absolute values. This technique drastically reduces noise in the difference due to drifts in the normalized flux arising from slow drifts in experimental parameters. In addition, the asymmetry formula suppresses systematic errors associated with these slow drifts. These two factors make it possible to achieve sub-ppm resolution in asymmetry measurements. In the following sections we will explore further the issues of statistical and systematic errors in an asymmetry measurement, but first we discuss how the helicity reversal scheme is designed to address these issues.

\subsubsection{Helicity Reversal Scheme}

The helicity reversal scheme is designed to avoid introducing spurious systematic contributions to the measured asymmetry and to avoid increasing the statistical error of the measurement. These goals are achieved by using a line-locked, pair-wise random helicity scheme described here.

We reduce sensitivity to $60 \mathrm{~Hz}$ line noise by reversing the helicity at a multiple of $1 / 60 \mathrm{~s}$ locked to the $\mathrm{AC}$ line. Integrating for a fixed time interval at a fixed phase relation to the $60 \mathrm{~Hz}$ wave keeps $60 \mathrm{~Hz}$ noise from increasing the statistical uncertainty of the measurement. We choose to integrate over two $60 \mathrm{~Hz}$ cycles, setting the helicity every $33.3 \mathrm{~ms}$. Each $33.3 \mathrm{~ms}$ period of constant helicity is called a window.

We reduce our sensitivity to other periodic noise in the environment by reversing the helicity randomly, so that the periodic noise cannot have any correlation with the helicity state of the beam. In this way any periodic noise effectively becomes random and averages to zero, avoiding a spurious systematic contribution to the 


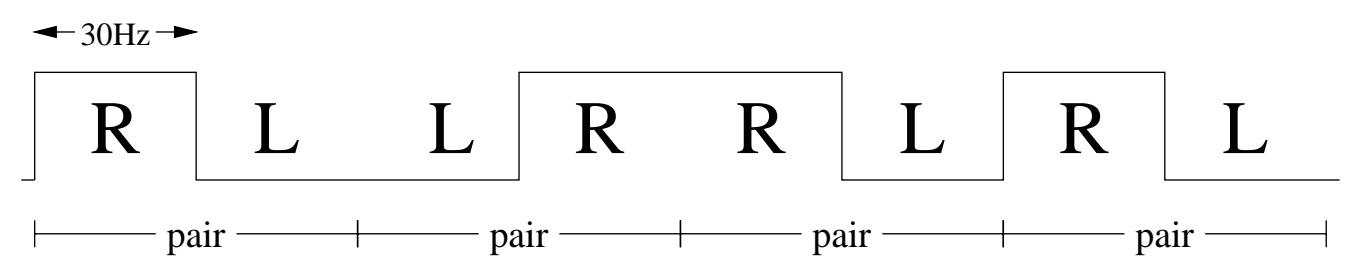

Figure 2.3: Sample helicity sequence. Line-locked 1/30 s helicity windows are grouped in a pair-wise random structure. The helicity of the first window of a pair is chosen randomly, and the second window is given the opposite helicity.

measured asymmetry.

But we cannot simply use a random helicity sequence if we also want to compute the asymmetry between adjacent windows of opposite helicity. In order to incorporate both requirements we impose a pair-wise random structure on the helicity sequence. The helicity of the first window of a pair is chosen randomly, and the second window is given the opposite helicity. A sample helicity sequence is shown in Figure 2.3.

\subsubsection{Statistical Error}

In order to measure the normalized flux, we integrate the analog signals from the two detectors and the beam current monitor over the duration of each helicity window. The normalized flux is given by the ratio of the integrated detector signal $D$ to the integrated current monitor signal $I$. Then we compute the raw asymmetry for each window pair:

$$
A_{\text {pair }}=\frac{\sigma_{R}-\sigma_{L}}{\sigma_{R}+\sigma_{L}}=\frac{D_{R} / I_{R}-D_{L} / I_{L}}{D_{R} / I_{R}+D_{L} / I_{L}} .
$$

We average all such pair asymmetries to obtain the final raw asymmetry. The

statistical error is given by $\sigma\left(A_{\text {pair }}\right) / \sqrt{N_{\text {pairs }}}$, where $\sigma\left(A_{\text {pair }}\right)$ is the RMS width of the distribution of pair asymmetries and $N_{\text {pairs }}$ is the total number of pairs 
The statistical distribution of pair asymmetries has a minimum RMS width $\sigma_{\min }\left(A_{\text {pair }}\right)$ determined by counting statistics of the scattering rate. If, on average, $N_{s}$ scattered electrons are detected per window pair, then

$$
\sigma_{\min }\left(A_{\text {pair }}\right)=\frac{1}{\sqrt{N_{s}}} .
$$

The rate estimate included below predicts that $\sigma_{\min }\left(A_{\text {pair }}\right)$ is $3600 \mathrm{ppm}$ for a beam current of $90 \mu \mathrm{A}$ at our kinematics. Instrumental noise in the measurement of either the scattered flux or beam current will broaden the width of the statistical distribution beyond this minimum width. We would like to keep this broadening negligible in order to achieve the smallest possible statistical error.

The key contributors to instrumental noise in the measurement of the scattered flux and beam current are ADC noise, BCM resolution, detector signal noise, and detector energy resolution. The first three contributions are each found to be less than $0.02 \%$, which is negligible compared with $\sigma_{\min }\left(A_{\text {pair }}\right)$. Detector energy resolution, discussed in Section 3.7, broadens $\sigma\left(A_{\text {pair }}\right)$ by only $\sim 1 \%$.

\section{Rate Estimate}

The Rosenbluth formula (Equation 1.6) gives the differential cross section for elastic electron-proton scattering. At our kinematics $\left(E=3.356 \mathrm{GeV}, \theta=12.3^{\circ}\right.$, and $\left.Q^{2}=0.479(\mathrm{GeV} / \mathrm{c})^{2}\right)$, the differential cross section is

$$
\left.\frac{d \sigma}{d \Omega}\right|_{\text {HAPPEX }} \approx 0.74 \text { microbarns/steradian. }
$$

The scattering rate $n_{s}$ seen by the detector is given by

$$
n_{s}=\frac{d \sigma}{d \Omega} \cdot I \cdot \rho L \cdot \Delta \Omega \cdot f,
$$

where $I$ is the beam current, $\rho$ and $L$ are the density and length of the target, $\Delta \Omega$ is the solid angle coverage of the detector, and $f$ is a correction factor due to 
Bremsstrahlung in the target. Using

$$
\begin{aligned}
\frac{d \sigma}{d \Omega} & =0.74 \mu \mathrm{b} / \mathrm{str}=7.4 \times 10^{-31} \mathrm{~cm}^{2} / \mathrm{str}, \\
\rho & =0.072 \mathrm{~g} / \mathrm{cm}^{3}=4.3 \times 10^{22} \text { protons } / \mathrm{cm}^{3}, \\
L & =15 \mathrm{~cm}, \\
\Delta \Omega & =5.5 \times 10^{-3} \mathrm{str}, \\
f & =0.8
\end{aligned}
$$

we have $n_{s}=2.1 \times 10^{-9} I$. That is, about one out of every 500 million electrons incident on our target will scatter into a given detector. Therefore at a beam current of $90 \mu \mathrm{A}$, the rates in each detector should be

$$
n_{s}=2.1 \times 10^{-9}\left(90 \times 10^{-6} \mathrm{C} / \mathrm{s}\right)\left(6.24 \times 10^{18} \text { electrons } / \mathrm{C}\right)=1.2 \mathrm{MHz} .
$$

Integrating over $32 \mathrm{~ms}$, each detector sees an average of 38,500 electrons per window and 77,000 electrons per window pair. Counting statistics dictate that the statistical distribution of pair asymmetries should have a minimum RMS width of

$$
\sigma_{\text {min }}\left(A_{\text {pair }}\right)=\frac{1}{\sqrt{77000}} \approx 3600 \mathrm{ppm} .
$$

The observed distribution of pair asymmetries has an RMS width of $\sim 3700 \mathrm{ppm}$ at a beam current of $90 \mu \mathrm{A}$.

\subsubsection{Systematic Error}

We would like to keep systematic errors at the level of $0.1 \mathrm{ppm}$ so that they are negligible compared with the statistical error. Although this level of systematic error is unreachable in an absolute cross section measurement, it is attainable in an asymmetry measurement because of the way the asymmetry is calculated. First of all, knowledge of absolute experimental parameters such as the absolute beam 
current and target density are not necessary since common scale factors cancel when the asymmetry is computed. Secondly, common-mode systematic errors in the measurement of the normalized fluxes $\sigma_{R}$ and $\sigma_{L}$ cancel to zeroth order when the difference is computed in the numerator, and contribute only to first order in the asymmetry when the sum is computed in the denominator, which we can see as follows.

Let $\Delta \sigma_{\text {sys }}$ be the (common-mode) systematic error in measuring the normalized flux. Then $\sigma_{R(L)}+\Delta \sigma_{\text {sys }}$ is the measured flux for electrons of positive (negative) helicity, and the measured asymmetry is

$$
A_{\text {meas }}=\frac{\left(\sigma_{R}+\Delta \sigma_{\text {sys }}\right)-\left(\sigma_{L}+\Delta \sigma_{\text {sys }}\right)}{\left(\sigma_{R}+\Delta \sigma_{\text {sys }}\right)+\left(\sigma_{L}+\Delta \sigma_{\text {sys }}\right)}=\frac{\sigma_{R}-\sigma_{L}}{\sigma_{R}+\sigma_{L}+2 \Delta \sigma_{\text {sys }}}
$$

A little algebra reveals that for $\Delta \sigma_{\text {sys }} \ll \sigma_{R} \approx \sigma_{L}$,

$$
A_{\text {meas }} \approx A_{L R}\left(1-\frac{\Delta \sigma_{\text {sys }}}{\sigma}\right)
$$

where $A_{L R}$ is the true asymmetry in the absence of systematic error and $\sigma=\left(\sigma_{R}+\right.$ $\left.\sigma_{L}\right) / 2$ is the average normalized flux. Thus we see that the fractional systematic error $\Delta \sigma_{s y s} / \sigma$ in measuring the normalized flux enters in proportion to the true asymmetry. For example, a 1\% systematic error in measuring the normalized flux contributes a systematic error to the measured asymmetry of $1 \%$ of the asymmetry. Hence asymmetry measurements are insensitive to small common-mode systematic errors in the measurement of the normalized flux, such as those associated with slow drifts of experimental parameters.

Of greater concern in asymmetry measurements are helicity-correlated systematic errors. These arise when there is a correlation between the beam helicity and experimental parameters such as beam energy or position. (That is, when it is not a mirror-image experiment.) Helicity-correlated systematic errors in the measurement 
of the normalized flux appear directly in the measured asymmetry, which we can see as follows.

Let $\Delta \sigma_{\text {sys }}^{\prime}$ be the helicity-correlated systematic error in the measurement of the normalized flux, so that the measured fluxes are given by $\sigma_{R}+\Delta \sigma_{\text {sys }}^{\prime}$ and $\sigma_{L}-\Delta \sigma_{\text {sys }}^{\prime}$. Then the measured asymmetry is

$$
A_{\text {meas }}=\frac{\left(\sigma_{R}+\Delta \sigma_{\text {sys }}^{\prime}\right)-\left(\sigma_{L}-\Delta \sigma_{\text {sys }}^{\prime}\right)}{\left(\sigma_{R}+\Delta \sigma_{\text {sys }}^{\prime}\right)+\left(\sigma_{L}-\Delta \sigma_{\text {sys }}^{\prime}\right)}=A_{L R}+\frac{\Delta \sigma_{\text {sys }}^{\prime}}{\sigma} .
$$

Thus we see that the fractional helicity-correlated systematic error $\Delta \sigma_{s y s}^{\prime} / \sigma$ appears directly in the measured asymmetry.

Because they are so potentially damaging to an asymmetry measurement, we make special efforts to deal with helicity-correlated systematics. In practice, the two major sources of helicity-correlated systematic errors are electronics crosstalk and helicity-correlated beam parameters. The special efforts made to deal with these are the topic of Section 2.3.

\section{Detector Linearity}

An important source of systematic error in flux-integrating asymmetry experiments is nonlinearity in the detectors measuring the scattered flux. The systematic error is dominated by the larger of the raw physics asymmetry or the intensity asymmetry, which we can demonstrate as follows.

The measured asymmetry of a window pair is given by Equation 2.2, which we repeat here for convenience:

$$
A_{\text {meas }}=\frac{D_{R} / I_{R}-D_{L} / I_{L}}{D_{R} / I_{R}+D_{L} / I_{L}} .
$$

Let us assume that there is a small nonlinearity in the detector response to the scattered flux, so that the detector response $D$ to the flux $F$ is given by

$$
D(F)=F+\epsilon F^{2},
$$


where $|\epsilon| \ll 1$. Here the nonlinearity is given by $\epsilon F$. The flux is taken to be proportional to the beam current $I$, with constant of proportionality given by the scattering cross section $\sigma$. Treating the two beam helicity states separately, we write

$$
F_{R, L}=\sigma_{R, L} I_{R, L}
$$

So we can express the detector response in terms of the beam current as

$$
D_{R, L}=\sigma_{R, L} I_{R, L}+\epsilon \sigma_{R, L}^{2} I_{R, L}^{2} .
$$

We use Equations 2.5-2.7 in Equation 2.4. A lot of algebra reveals that to leading order in the asymmetries, the measured asymmetry in the presence of a detector nonlinearity $X \equiv \epsilon F$ is given by

$$
A_{\text {meas }}=A_{L R}+X\left(A_{L R}+A_{I}\right),
$$

where $A_{I}$ and $A_{L R}$ are the intensity and raw physics asymmetries, respectively, given by

$$
A_{I}=\frac{I_{R}-I_{L}}{I_{R}+I_{L}} \quad \text { and } \quad A_{L R}=\frac{\sigma_{R}-\sigma_{L}}{\sigma_{R}+\sigma_{L}} .
$$

This result shows that the systematic error due to detector nonlinearity scales in proportion to the larger of the physics asymmetry or the intensity asymmetry. We draw two conclusions:

1. In order to minimize the systematic error due to the intensity asymmetry, it is sufficient to keep the intensity asymmetry below the level of the physics asymmetry.

2. Detector linearity is a concern for asymmetry measurements in which the scattered flux is integrated because there is an inherent fractional systematic error equal to the nonlinearity. 
The intensity asymmetry is kept below the level of the raw physics asymmetry by a feedback system, described in Section 2.3.2. Since the expected raw asymmetry is $\sim 6 \mathrm{ppm}$, detector nonlinearity of $1 \%$ will contribute a systematic error less than $0.1 \mathrm{ppm}$.

\subsection{Helicity-Correlated Systematics}

We would like to keep systematic errors at the level of $0.1 \mathrm{ppm}$ so that they are negligible compared with the statistical error in the measurement of the raw asymmetry. One key to suppressing systematic errors is to maintain negligible correlations between the helicity of the electron beam and any other properties of the beam, such as intensity, energy, position, or angle. In this section we describe a feedback system used to minimize helicity-correlated intensity differences and a beam modulation technique used to evaluate and correct for helicity-correlated position, angle, and energy differences. Another class of helicity-correlated systematic is electronics crosstalk, which was suppressed by delaying transmission of the helicity signal and by using a method of passive helicity reversal.

\subsubsection{Electronic Crosstalk}

Since we are trying to measure the helicity-correlated difference between two electronic signals to a precision of $1 \mathrm{ppm}$, it is important that the measurement not be contaminated by electronic crosstalk with signals carrying information about the helicity of the beam. In order to achieve maximum insensitivity to crosstalk, we did not permit any in-time helicity signal outside the vicinity of the polarized source. Instead a delayed version of the helicity signal was sent to the DAQ system, and the helicity for each window was aligned correctly during data analysis. 
We carried out a crosstalk test throughout the production run: The signals from three $1.5 \mathrm{~V}$ batteries were integrated by our DAQ system and the average helicitycorrelated asymmetry was computed for each battery. Nonzero asymmetries would indicate crosstalk in the measurement electronics. Table 2.1 shows the average helicity-correlated asymmetry for each battery measured over the entire production run. All measurements are consistent with zero. This is an encouraging result, but does not settle the issue of electronics crosstalk.

\begin{tabular}{|c|c|}
\hline & Asymmetry (ppm) \\
\hline vbatt1 & $-0.005 \quad \pm \quad 0.007$ \\
\hline ibatt1 & $-0.001 \pm 0.015$ \\
\hline ibatt2 & $0.005 \pm 0.016$ \\
\hline
\end{tabular}

Table 2.1: Battery asymmetries.

Systematic effects due to electronics crosstalk are suppressed using a technique of passive helicity reversal. As described in Section 3.2, by inserting a half-wave plate into the laser beam just upstream of the Pockels cell, we can reverse the correlation between the electronic helicity signals and the actual helicity of the electron beam. Thus asymmetry measurements made while the half-wave plate is inserted will have a systematic error due to electronic crosstalk which is opposite that when the halfwave plate is removed from the laser beam. We take an equal amount of production data in each half-wave plate configuration, and averaging the results of the two data sets then cancels the systematic effect due to electronics crosstalk.

\subsubsection{Intensity Differences}

Although helicity-correlated intensity differences are normalized out when computing the raw pair asymmetry (see Equation 2.2), there are two reasons to minimize 
the average intensity difference between the two polarization states of the electron beam:

1. Intensity differences induce differences in other beam parameters: The accelerator turns intensity differences into energy differences through beam loading, and energy differences become position differences due to achromatic transport through the machine.

2. As discussed in Section 2.2.3, nonlinearity in the detectors measuring the scattered flux introduce a systematic error in the asymmetry measurement which is proportional to the helicity-correlated intensity difference.

Beam loading is demonstrated in Section 4.3. Since the effects of beam loading do not become appreciable until the intensity asymmetry reaches $100 \mathrm{ppm}$, the more immediate problem is detector nonlinearity. As discussed in Section 2.2.3, keeping the intensity asymmetry less than the raw physics asymmetry of $6 \mathrm{ppm}$ is sufficient.

Preliminary polarized beam tests conducted in March 1997 showed helicitycorrelated intensity differences which varied from 20 to $100 \mathrm{ppm}$. We employed a feedback scheme during the production run to reduce the intensity asymmetry to acceptable levels. The feedback system will be described in this section.

Intensity differences in the electron beam originate with the circularly polarized laser beam striking the photocathode. We minimize the electron beam intensity differences by using the so-called polarization-induced transport asymmetry (PITA) effect to control the intensity asymmetry of the laser beam at the photocathode. Before describing the feedback system, we first describe the PITA effect. 


\section{PITA Effect}

When linearly polarized light strikes a refracting surface at an angle, the electric field vector can be resolved into components parallel and transverse to the plane of incidence, traditionally written $E_{p}$ and $E_{s}$. It is well known that these components have different transmission and reflection coefficients which depend on the angle of incidence. Thus the total transmission coefficient of linearly polarized light is a function of the angle of incidence as well as the angle between the polarization vector and the plane of incidence. Similarly, elliptically polarized light, which can be separated into perpendicular linear components which are out of phase with one another, has a transmission coefficient which depends on the angular orientation of the ellipse's major and minor axes with respect to the plane of incidence. If the elliptical polarization were perfectly circular, there would be no such angular dependence.

Although the laser light exiting the Pockels cell is supposed to be circularly polarized, in practice it is always slightly elliptical and therefore is transmitted differently through a non-normal refracting surface depending on the degree and orientation of the ellipticity. Since the degree and orientation of the ellipticity may be different for the left- and right-handed beams, there is, in general, an asymmetry in laser intensity downstream of any optical element. This effect is called the polarizationinduced transport asymmetry (PITA) effect. The intensity asymmetry due to the PITA effect is given by

$$
A_{P I T A}=\frac{I_{R}-I_{L}}{I_{R}+I_{L}},
$$

where $I_{R}\left(I_{L}\right)$ is the intensity of the right-handed (left-handed) beam.

The PITA effect may be described mathematically as follows $[12,13]$. In order to produce circularly polarized light, the Pockels cell must introduce a retardation 
of exactly $\pm \pi / 2$; otherwise elliptical polarization will be produced. Let $\theta_{+}$be the retardation introduced by the Pockels cell for one helicity, and let $\theta_{-}$be the retardation for the opposite helicity. General variations about the nominal retardations of $\pm \pi / 2$ may be parameterized by introducing a symmetric offset $\alpha$ and antisymmetric offset $\Delta$ :

$$
\begin{aligned}
& \theta_{+}=+\left(\frac{\pi}{2}+\alpha+\Delta\right), \\
& \theta_{-}=-\left(\frac{\pi}{2}+\alpha-\Delta\right) .
\end{aligned}
$$

Now assume the elliptically-polarized laser beam strikes an optical element which has different transmission coefficients $T_{x}$ and $T_{y}$ along orthogonal axes $x$ and $y$ transverse to the beam. This optical element might be a window or mirror which is not perfectly normal to the beam. We define the transmission asymmetry $\epsilon$ for this optical element to be

$$
\epsilon=\frac{T_{x}-T_{y}}{T_{x}+T_{y}} .
$$

Finally, let $\Theta$ be the angle between the major axis of the ellipse and the transmission axis of the optical element. It can be shown that for small variations of the Pockels cell retardation about $\pm \pi / 2$, the resulting intensity asymmetry is proportional to the antisymmetric offset $\Delta$ :

$$
A_{P I T A}=2 \epsilon \Delta \sin (2 \Theta) \text {. }
$$

This is the desired result. By manipulating $\Delta$, we can control the helicity-correlated intensity asymmetry of the laser beam. The following section will describe how we used the PITA effect as the basis for a feedback system to eliminate the intensity asymmetry. 


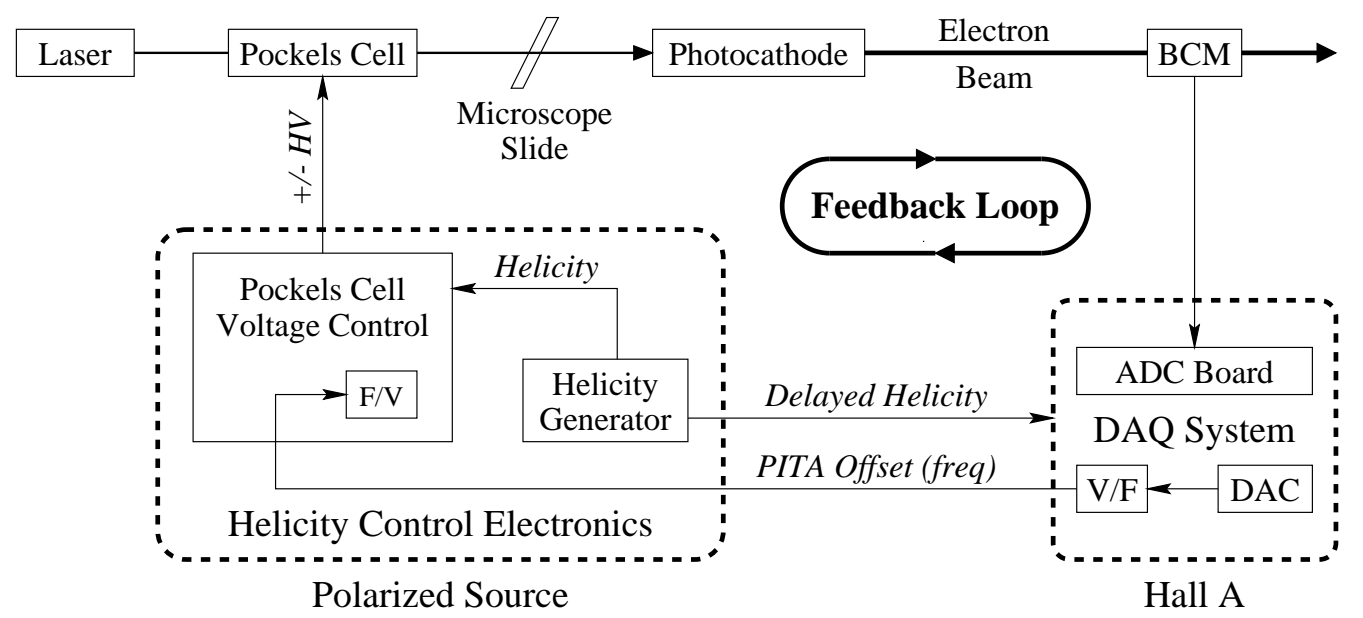

Figure 2.4: Feedback system.

\section{Feedback System}

A diagram of the feedback system is shown in Figure 2.4. We placed a microscope slide downstream of the Pockels cell and cocked it about $20^{\circ}$ with respect to the laser beam normal in order to generate a sizable transmission asymmetry $\epsilon$ which dominated all other sources. The Pockels cell voltage control electronics are capable of applying a voltage offset $V_{\Delta}$ antisymmetrically to the nominal quarter-wave voltages $\pm V_{\lambda / 4}$ for the Pockels cell. That is, the Pockels cell voltages $V_{ \pm}$are given by

$$
\begin{aligned}
& V_{+}=+\left(V_{\lambda / 4}+V_{\Delta}\right) \\
& V_{-}=-\left(V_{\lambda / 4}-V_{\Delta}\right)
\end{aligned}
$$

(compare with Equations 2.8 and 2.9). The size of the offset $V_{\Delta}$ is controlled by the PITA Offset signal generated by the DAQ system monitoring the intensity asymmetry in Hall A.

The intensity asymmetry is controlled as follows. Let $A_{I}$ be the helicity-correlated intensity asymmetry of the electron beam measured in Hall A. Let $A_{P I T A}$, given by 


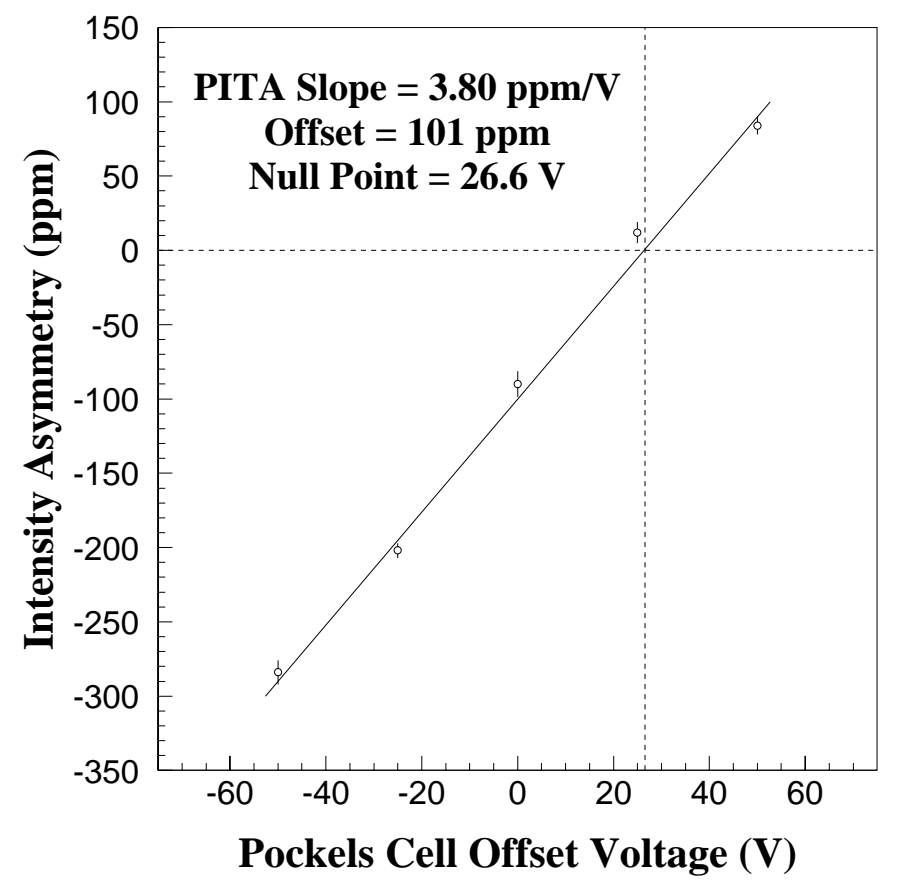

Figure 2.5: PITA curve.

Equation 2.10 , be the contribution to $A_{I}$ due to the microscope slide and let $A_{0}$ represent the contribution from all other sources. Then

$$
A_{I}=A_{0}+A_{\text {PITA }}=A_{0}+m V_{\Delta},
$$

where $m=2 \epsilon \sin (2 \Theta)$. Figure 2.5 is a plot of $A_{I}$ measured at five different settings of the voltage offset $V_{\Delta}$. We see that $A_{I}$ and $V_{\Delta}$ are linearly related, as predicted by Equation 2.11. We call this plot a PITA curve and refer to the slope $m$ of the linear fit as the PITA slope. The overall intensity asymmetry $A_{I}$ can be made zero by adjusting $V_{\Delta}$ so that

$$
V_{\Delta}=-\frac{A_{0}}{m} \equiv V_{0},
$$

where $V_{0}$ is called the null point.

One might think that we could simply take a PITA curve, calculate the voltage 
offset which nulls the intensity asymmetry, set the voltage offset to the null point, and sit there forever. There are two problems with this strategy, however:

1. Because of beam intensity noise, the null point cannot be found with infinite precision, so the intensity asymmetry will converge to a nonzero value.

2. The null point drifts, we find empirically.

Thus we must continuously feedback on the PITA slope to successfully null the intensity asymmetry.

\section{Feedback Implementation}

The feedback scheme is implemented as follows. First a PITA curve is measured as in Figure 2.5, and the slope $m$ and approximate null point are noted. The Pockels cell voltage offset $V_{\Delta}$ is set to the approximate null point. Then a production run

is started, and the intensity asymmetry is monitored throughout the run. The run is divided into fixed intervals called miniruns. At the end of each minirun, a correction to the Pockels cell voltage offset is calculated based on the average intensity asymmetry during the minirun just completed. The voltage offset $V_{\Delta}$ is set to the corrected value, and the cycle is repeated indefinitely.

The scheme may be modeled mathematically as follows. Let us assume that the PITA curve has constant slope $m$ and null point $V_{0}$. That is, the true intensity asymmetry as a function of the Pockels cell voltage offset $V_{\Delta}$ is given exactly by

$$
A_{I}=m\left(V_{\Delta}-V_{0}\right) .
$$

Let $A_{i}$ be the intensity asymmetry measured during the $i$ th minirun, and let $\delta_{i}$ be the deviation of the measured intensity asymmetry from the true intensity asymmetry due to beam intensity noise. Finally, assume that $V_{1} \neq V_{0}$ is the voltage offset for 
the first minirun, so that the true intensity asymmetry during the first minirun is given by $A_{0} \equiv m\left(V_{1}-V_{0}\right)$.

Then the intensity asymmetry measured during the first minirun is

$$
A_{1}=A_{0}+\delta_{1}
$$

We adjust the Pockels cell voltage for the $(i+1)$ th minirun based on the intensity asymmetry measured during the $i$ th minirun according to the formula

$$
V_{i+1}=V_{i}-\frac{A_{i}}{m} .
$$

That is, we feedback on the PITA slope to introduce a voltage offset which compensates for the asymmetry measured during the previous minirun. Using Equations 2.13 and 2.14 in Equation 2.12, we see that the actual intensity asymmetry for the second minirun will be $-\delta_{1}$. Thus the feedback algorithm has corrected for the initial uncertainty in the null point. But due to intensity noise, the measured intensity asymmetry is $A_{2}=-\delta_{1}+\delta_{2}$. In general, the measured intensity asymmetry for the $i$ th minirun is

$$
A_{i}=-\delta_{i-1}+\delta_{i}
$$

The cumulative measured intensity asymmetry over $\mathrm{N}$ miniruns is then a telescoping sum:

$$
\begin{aligned}
\left\langle A_{i}\right\rangle_{N} & =\frac{\left(A_{0}+\delta_{1}\right)+\left(-\delta_{1}+\delta_{2}\right)+\ldots+\left(-\delta_{N-1}+\delta_{N}\right)}{N} \\
& =\frac{A_{0}+\delta_{N}}{N}=\frac{A_{0}}{N} \pm \frac{\sigma}{N}
\end{aligned}
$$

where $\sigma^{2}=\left\langle\delta_{i}^{2}\right\rangle$.

We see that as $N$ increases, the cumulative average intensity asymmetry, which is dominated by the initial uncertainty in the null point, converges to zero like $1 / N$. The algorithm basically involves feeding intensity noise back into the system. A side 
effect is to make the measured intensity asymmetry converge to the average value like $\sigma / N$ rather than $\sigma / \sqrt{N}$ as would otherwise happen. So even if the null point could be measured with infinite precision (so that $A_{0}=0$ ), there is a benefit to continuous feedback. And if the null point drifts over time, the intensity asymmetry will still converge to zero as long as the drift between miniruns is much less than the intensity noise over a minirun:

$$
\left\langle\left(A_{i}-A_{i+1}\right)^{2}\right\rangle \ll \sigma^{2} .
$$

\section{Parameters of Feedback Scheme}

There are two parameters in the feedback scheme which may be adjusted:

1. the PITA slope, and

2. the minirun length.

We set the PITA slope to $\sim 3 \mathrm{ppm} / \mathrm{V}$ in advance of the production run by adjusting the angle of the microscope slide. The PITA slope must be re-measured every time the half-wave plate is inserted or removed from the laser beam and also after the laser spot is moved on the photocathode. Otherwise the PITA slope is observed to remain essentially constant. Even if the PITA slope drifts during a feedback sequence, the feedback scheme converges as long as the slope is not wrong by more than a factor of two.

The minirun length was set so that the intensity asymmetry during each minirun could be measured with a statistical error $\sigma$ less than the predicted raw physics asymmetry of $6 \mathrm{ppm}$. The window-to-window intensity jitter was typically less than $250 \mathrm{ppm}$. In this case we set the minirun length to 2500 window pairs, yielding

$$
\sigma=\frac{250 \mathrm{ppm}}{\sqrt{2500}}=5 \mathrm{ppm} .
$$


The minirun length was increased at certain times during the production run when the intensity noise was larger.

Since the feedback scheme causes the intensity asymmetry to hug zero with variations given by $\sigma$, adopting this standard means that typical average minirun intensity asymmetries will be below the level of the physics asymmetry, which ensures that the feedback will have no adverse effect on the systematic or statistical error of the asymmetry measurement. For our experiment this was a reasonable goal, since the beam intensity and monitors were so quiet and the PITA slope and offset were relatively stable. A discussion of the tradeoffs among feedback parameters in a more hostile environment can be found in the references [13].

The feedback system proved to be very successful in reducing the beam intensity asymmetry. The overall performance of the feedback scheme, as well as a demonstration of $1 / N$ convergence, is shown in Section 4.3.

\subsubsection{Position, Angle, and Energy Differences}

Because the electron-proton scattering cross section depends on energy and scattering angle, helicity-correlated differences in the position, angle, or energy of the electron beam at the target can cause a "false" asymmetry in the normalized flux. We evaluate this source of systematic error using a beam modulation technique which allows us to determine the false component of the measured asymmetry and apply a correction to the measured asymmetry if necessary.

In order to determine the size of the false asymmetry, we must know how the normalized flux varies with the energy, angle, and position of the electron beam. The sensitivity of the normalized flux to beam parameters depends on many factors, including the geometry of the target, spectrometers, and detectors, as well as the energy and angular dependence of the differential scattering cross section. Our 
plan is to measure both the first-order sensitivity $\partial \sigma / \partial x_{i}$ of the normalized flux to changes in beam parameters $x_{i}$ and the average helicity-correlated differences $\Delta x_{i}$ in these beam parameters. Then the false component of the measured asymmetry is given by

$$
A_{\text {false }}=\frac{1}{2 \sigma} \sum_{i} \frac{\partial \sigma}{\partial x_{i}} \Delta x_{i},
$$

where $\sigma=\left(\sigma_{R}+\sigma_{L}\right) / 2$ is the average normalized flux.

We determined the sensitivity of the measured cross section to beam parameters by perturbing the beam in a controlled manner and measuring the response of the measured cross section. The beam position was perturbed by magnetic coils placed along the beamline, resulting in small changes in the position and angle of the beam at the target. The beam energy was perturbed by an energy vernier installed on the final cryomodule in the south linac. The transverse horizontal and vertical beam position was measured by two beam position monitors (BPMs) near the target, and the relative beam energy was measured by a horizontal BPM located in the bend in the Hall A beamline near the point of maximum energy dispersion. Figure 2.6 shows the locations of the monitors and coils along the Hall A beamline. There are a total of seven steering coils. Four $X$ coils modulate the beam in the horizontal direction and three $Y$ coils modulate the beam vertically.

The steering coils should perturb the beam with an amplitude large enough so that the effect of the perturbation on the beam position can be seen above the normal beam jitter, but small enough that changes to the scattering rates observed in the detectors are negligible compared with the fluctuations due to counting statistics. Also, the beam position should be varied either very slowly or only between window pairs so that it is essentially constant over each window pair. If these criteria can be met, then the beam modulation is effectively a helicity-uncorrelated slow drift and the measurement of the sensitivity can be carried out while taking production 


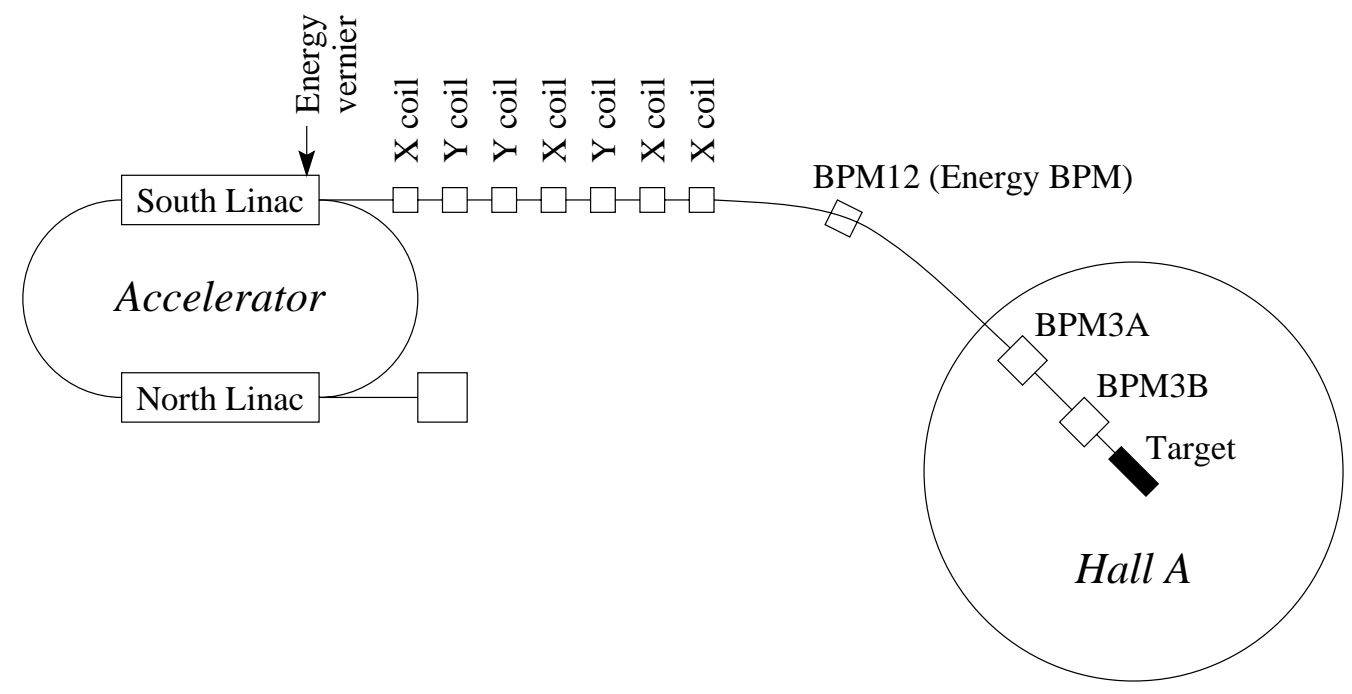

Figure 2.6: Diagram showing the locations of the beam position monitors and steering coils along the Hall A beamline.

data without adversely affecting the physics measurement.

Figure 2.7 shows actual beam measurement data taken over one beam modulation cycle during the production run. Results of the beam modulation analysis are presented in Section 4.4 . 


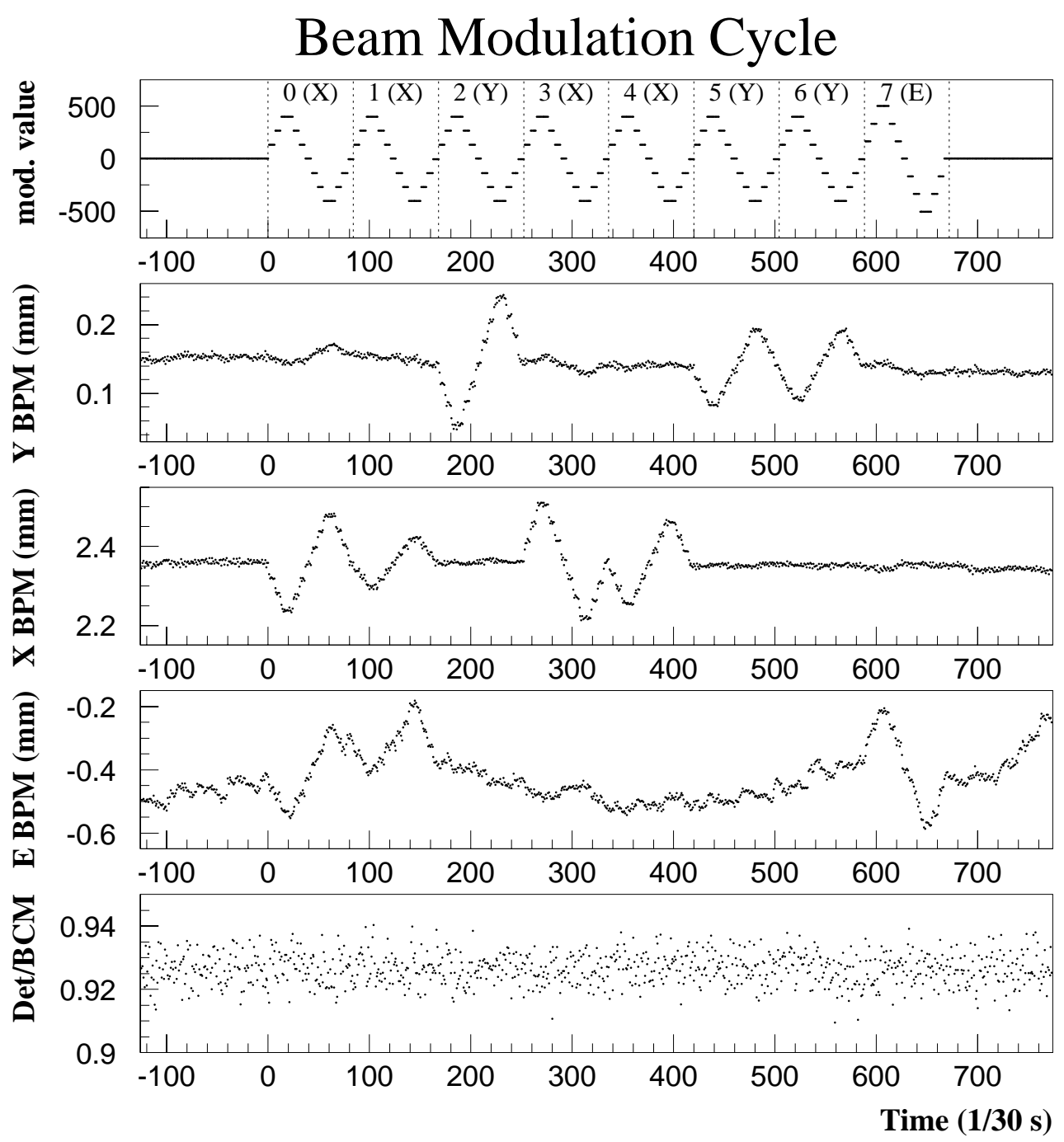

Figure 2.7: The top plot illustrates the modulation cycle: Each of the seven coils is varied one at a time in succession. The current in each coil is ramped up, down, and back up again in discrete steps over one period in a sawtooth pattern. Finally the energy vernier is varied in a similar pattern. The middle three plots show the horizontal and vertical beam position measured by BPM3a and the beam energy as measured by BPMx 12 . The horizontal (vertical) beam position is seen to respond when the $X(Y)$ coils are ramped, and the beam energy is seen to respond when the energy vernier is ramped. The bottom plot shows the normalized detector flux. Changes in its average value are not visible above the statistical fluctuations, as required. 


\section{Chapter 3}

\section{Description of the Apparatus}

\subsection{Accelerator}

The Continuous Electron Beam Accelerator Facility at Jefferson Lab is a recirculating linear electron accelerator capable of delivering up to $200 \mu \mathrm{A}$ of continuous-wave (CW) electron beam split simultaneously among three experimental halls. The accelerator is laid out in a racetrack configuration, as shown in Figure 3.1. Electrons are injected into the main accelerator loop at an energy of $45 \mathrm{MeV}$. They gain up to $400 \mathrm{MeV}$ in the north linac, are bent $180^{\circ}$ by magnets in the recirculating arcs, and gain another $400 \mathrm{MeV}$ in the south linac. Up to five passes around the machine are possible, resulting in a maximum beam energy of approximately $4 \mathrm{GeV}$.

Acceleration is provided by superconducting radio-frequency (rf) cavities operating at $1497 \mathrm{MHz}$. The resulting electron beam has microstructure consisting of bunches of electrons at this frequency. After each pass around the accelerator, an individual electron bunch may either be extracted and sent to one of the three experimental halls or be sent around the accelerator again (to a maximum of five passes). This allows each experimental hall to receive a different beam energy. When all three halls are operating, each hall gets every third electron bunch, so the microstructure seen in the hall is $499 \mathrm{MHz}$. 


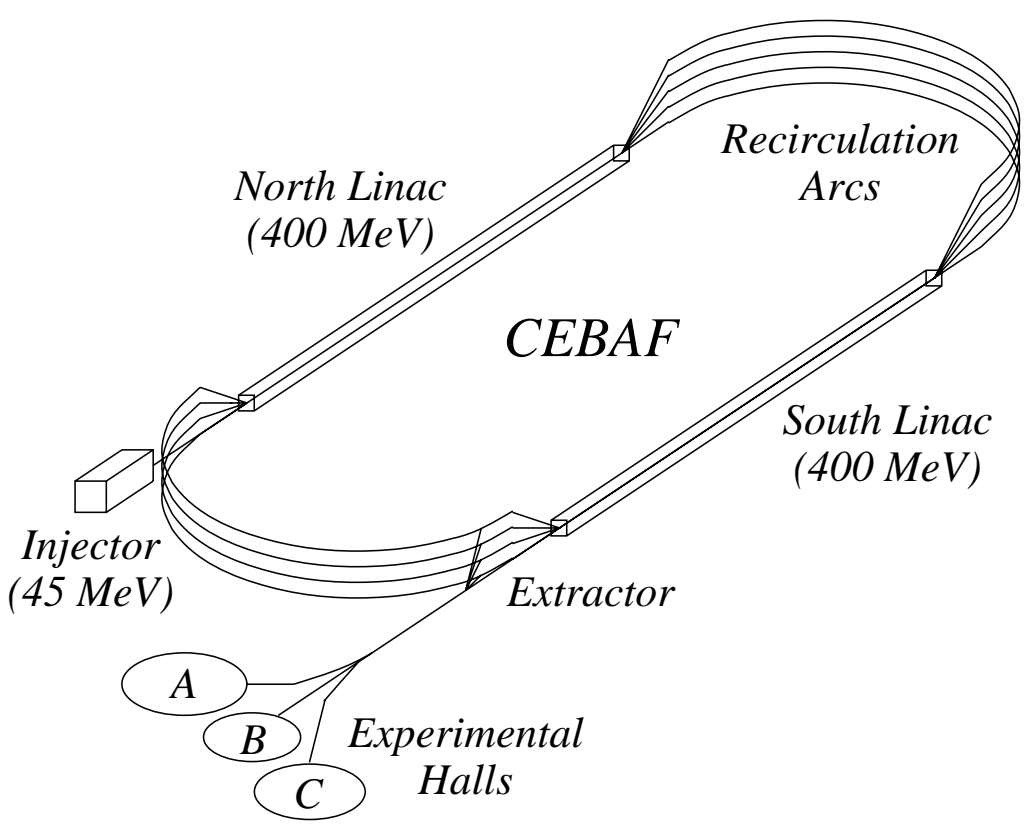

Figure 3.1: Continuous Electron Beam Accelerator Facility at Jefferson Laboratory.

Polarized electrons originate in a bulk GaAs photocathode excited by circularly polarized laser light, yielding a beam polarization of approximately 40\%. A Wien filter in the injector sets the launch angle of the polarization vector in order to compensate for $g-2$ precession in the bending arcs and provide longitudinal polarization at the experimental hall.

\subsection{Polarized Source Laser}

The function of the source laser is to provide circularly polarized laser light with $1497 \mathrm{MHz}$ microstructure to the photocathode. A diagram of the source laser system is shown in Figure 3.2. There are actually three lasers, one for each experimental hall. The use of three lasers allows the laser intensity and therefore the electron beam intensity to be varied independently for each hall. Each laser consists of a gainswitched diode seed laser and a single-pass diode optical amplifier [14, 15, 16]. Gain 


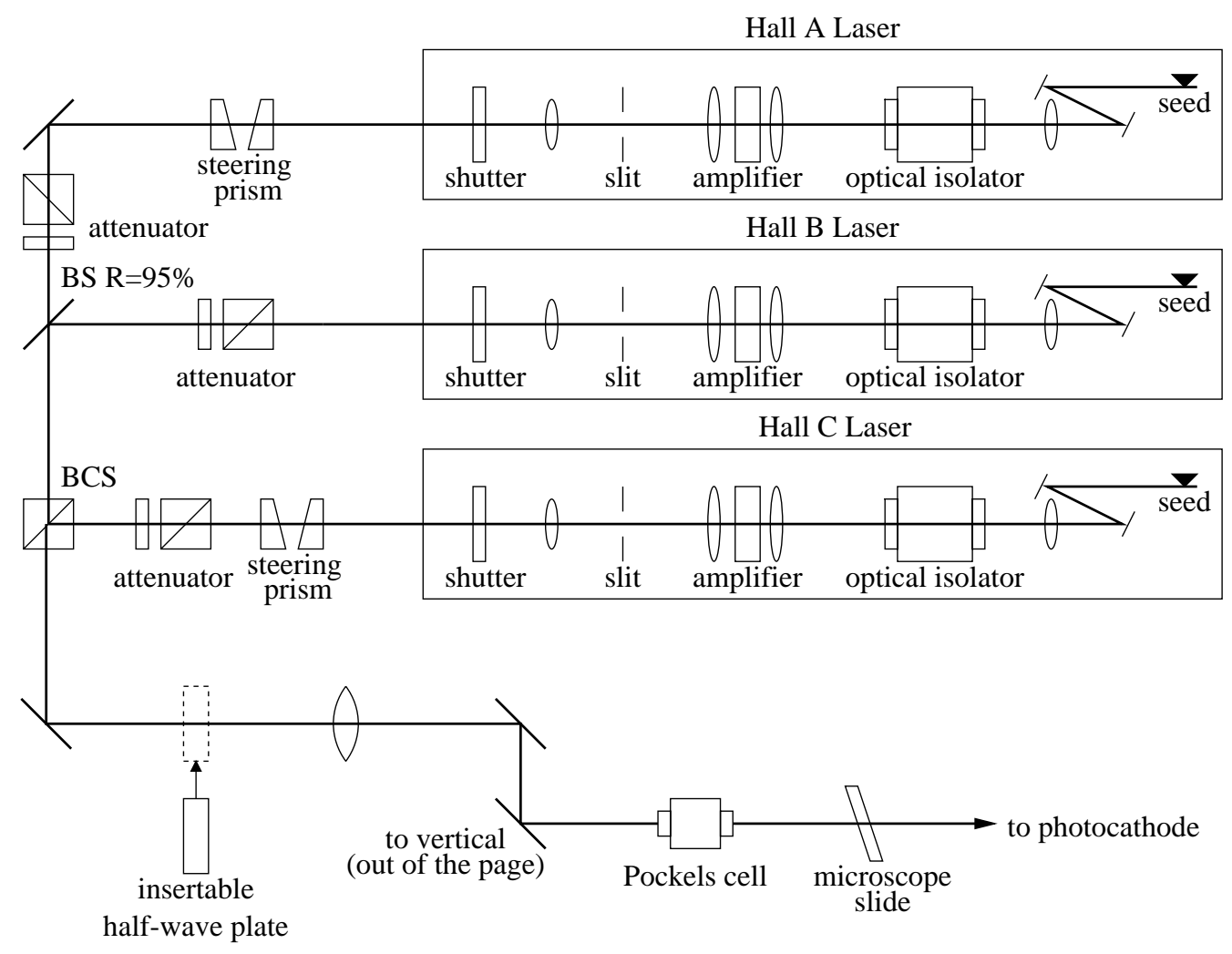

Figure 3.2: The polarized source laser.

switching is used to generate pulsed laser light at the accelerating cavity operating frequency. Each seed laser is driven at $499 \mathrm{MHz}, 120^{\circ}$ out of phase with one another. The three beams are combined to produce a $1497 \mathrm{MHz}$ pulse train.

The light from the seed laser passes through an optical isolator and then is focussed into the diode optical amplifier. Since the amount of amplification is a function of the diode amplifier's drive current, the pulse intensity for each hall's laser may be controlled independently. The pulsewidth out of the diode optical amplifier is typically $60-80$ ps.

The pulsed light from each laser passes through an attenuator consisting of a rotatable half-wave plate followed by a fixed linear polarizer. The attenuator serves as an additional intensity control for each laser. Beam-combining optics align the 
three laser beams on top of one another. The combined beam enters a Pockels cell, which is basically a voltage-controlled wave plate. We use it as a $\pm \lambda / 4$ (quarterwave) plate to convert the linearly polarized light into left- or right-handed circularly polarized light. A lens placed upstream of the Pockels cell focuses the laser beam onto the photocathode.

For our experiment, a microscope slide was inserted in the beam downstream of the Pockels cell in order to use the PITA effect to null the helicity-correlated intensity asymmetry, as described in Section 2.3.2. In addition, a remotely-controlled $\lambda / 2$ (half-wave) plate which could be inserted into or removed from the laser beam was placed just upstream of the Pockels cell. The $\lambda / 2$ plate is aligned with its fast axis at $45^{\circ}$ with respect to the linear polarization of the laser beam, so that it rotates the incoming linear polarization by $90^{\circ}$ and switches the handedness of the circular polarization exiting the Pockels cell.

\subsection{Helicity Control Electronics}

Because of the way polarized electrons are generated, the task of setting the helicity of the beam is reduced to the task of controlling the circular polarization of the source laser. A diagram of the helicity control electronics is shown in Figure 3.3. A detailed description of each module including circuit diagrams is given in the Appendix. Here we briefly highlight the purpose and function of each of the components.

Circularly polarized laser light is generated from linearly polarized laser light using a Pockels cell. The Pockels cell is aligned so that $\pm \lambda / 4$ retardation yields either right- or left-circular polarization. The HV switcher provides the Pockels cell with either positive or negative high voltage depending on the state of a digital control signal. The programmable $\pm \mathrm{HV}$ supplies are set to supply voltages corresponding 


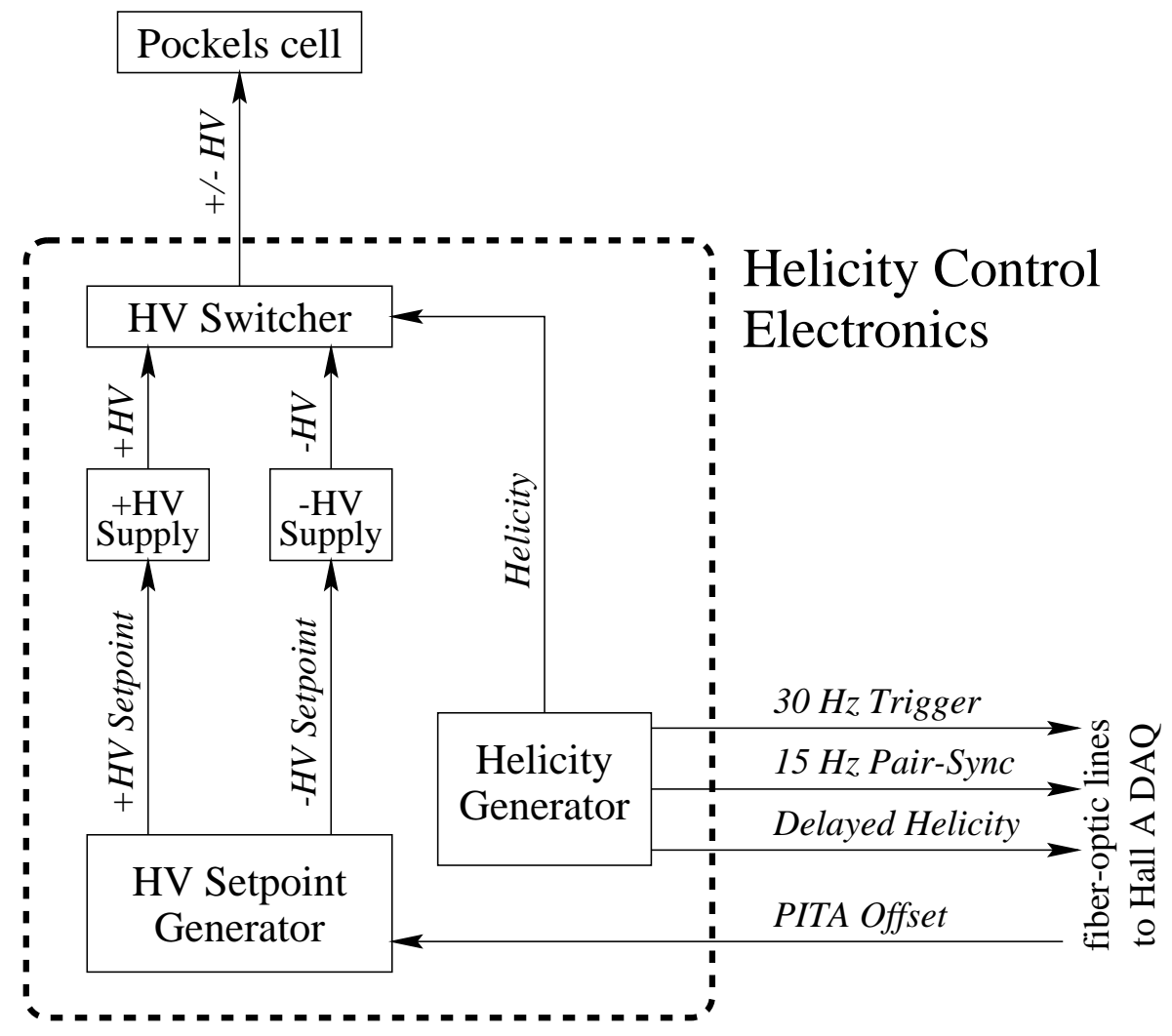

Figure 3.3: Helicity control electronics.

to $\pm \lambda / 4$ retardation, which is approximately $\pm 2.5 \mathrm{kV}$. The net effect of the entire system is that the helicity of the electron beam depends on the state of the digital control signal, or Helicity signal.

The Helicity signal is provided by the Helicity Generator, which thereby controls the helicity sequence and timing structure of the polarized electron beam. It generates the line-locked, pair-wise pseudorandom helicity sequence described in Section 2.2.1. In addition to the Helicity signal, it produces three other signals which are sent to the HAPPEX DAQ system in Hall A: The $30 \mathrm{~Hz}$ Trigger marks the beginning of a helicity window and tells the DAQ when to start integrating. The Delayed Helicity and $15 \mathrm{~Hz}$ Pair-Sync signals give information about the beam helicity and allow the correct helicity to be assigned to each window during data 

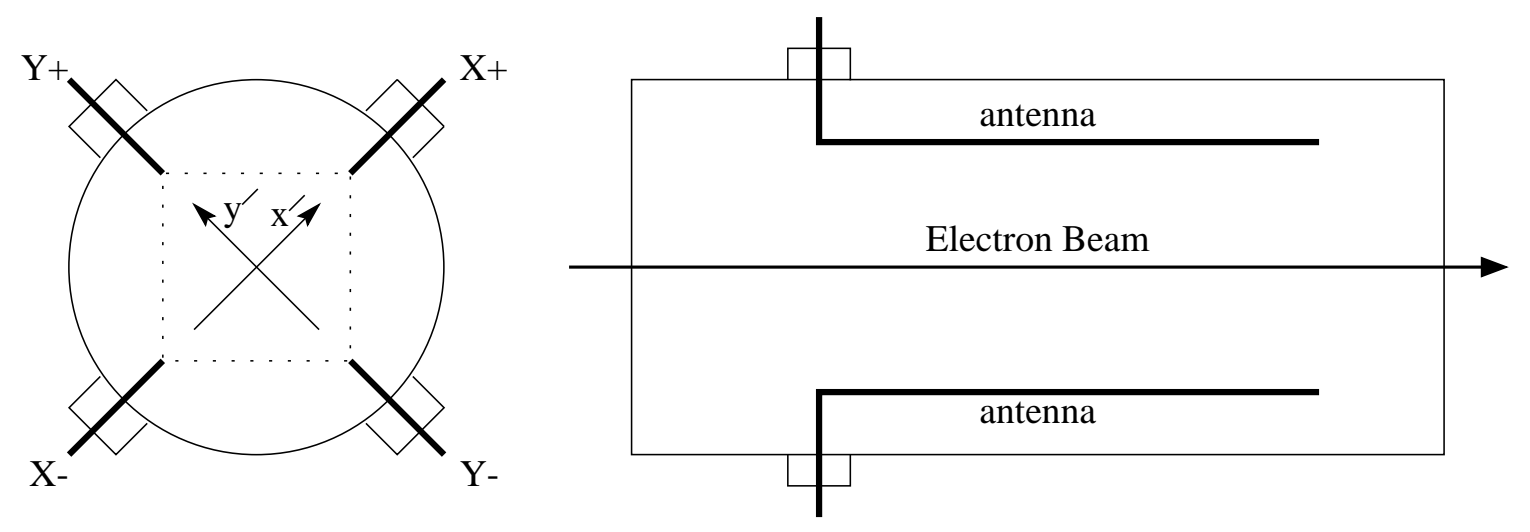

Figure 3.4: Diagram of beam position monitor.

analysis. The Helicity signal is not sent outside the helicity control electronics in order to avoid electronics crosstalk, as discussed in Section 2.3.1.

The system is complicated somewhat by the implementation of intensity feedback, which requires the ability to introduce a small antisymmetric offset to the Pockels cell quarter-wave voltages, as discussed in Section 2.3.2. This requirement is incorporated into the HV Setpoint Generator, which supplies the programming voltages to the HV supplies. The size of the voltage offset is determined by the PITA Offset signal provided by the HAPPEX DAQ system in Hall A.

\subsection{Beam Monitors}

Quiet and sensitive beam monitors are necessary for measuring the beam current used in the calculation of the measured raw asymmetry and for monitoring of helicity-correlated beam differences. 


\subsubsection{Beam Position Monitors}

The beam position monitors (BPMs) are thin wire stripline devices consisting of four quarter-wavelength wire antennas oriented parallel to the electron beam and located at the corners of a square as shown in Figure $3.4[17,18]$. The rf signal from each antenna is processed electronically to yield a DC signal which is proportional to the beam current times the distance between the beam and the antenna. These DC signals, labeled $X^{+}, X^{-}, Y^{+}$, and $Y^{-}$, are integrated by our DAQ system. A current-independent measure of the beam position is obtained by taking the difference divided by the sum for each pair of antennas:

$$
x_{\text {beam }}^{\prime}=\frac{X^{+}-X^{-}}{X^{+}+X^{-}}, \quad y_{\text {beam }}^{\prime}=\frac{Y^{+}-Y^{-}}{Y^{+}+Y^{-}} .
$$

A $45^{\circ}$ coordinate rotation from the primed coordinate system $\left(x^{\prime}, y^{\prime}\right)$ may then be performed to give the beam position in horizontal and vertical lab coordinates $(x, y)$.

Since the antenna signals vary with beam current, gain switching is used to keep the DC output signals of approximately constant size ( $\sim 4$ volts) regardless of the beam current. The BPM may be used as a crude current monitor by turning gain switching off.

BPM resolution may be studied by measuring the beam position at several points along the beamline and using the measurements from neighboring BPMs to predict the position at a given BPM. The BPM resolution is given by the residuals of the correlation between the predicted and measured position. Figure 3.5 shows the results of such a study. The BPM resolution is determined to be better than $1 \mu \mathrm{m}$ integrated over one $32 \mathrm{~ms}$ helicity window at a beam current of $100 \mu \mathrm{A}$. 


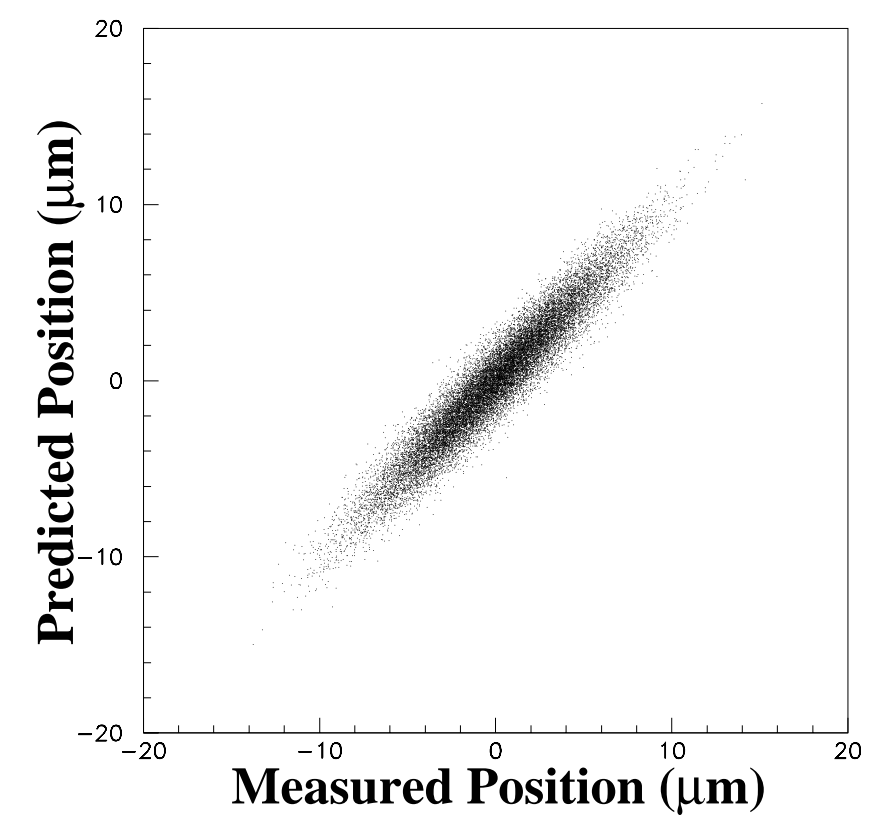

Figure 3.5: A plot showing BPM resolution. Window-to-window beam jitter, as measured by a particular BPM, is plotted along the x-axis, and the beam position as predicted by neighboring BPMs is plotted along the $\mathrm{y}$-axis. The residuals of the correlation demonstrate the BPM resolution to be better than $1 \mu \mathrm{m}$.

\subsubsection{Beam Current Monitors}

There are two beam current monitors (BCMs), both located in Hall A 24 meters upstream of the target. Each BCM is a cylindrical resonant cavity tuned to the machine frequency of $1497 \mathrm{MHz}$. A loop antenna couples rf power out of the cavity, giving a signal which depends on the beam current. This $1497 \mathrm{MHz}$ signal is downconverted to $1 \mathrm{MHz}$ and sent upstairs to the Hall A counting house. An RMS-to-DC converter located in the counting house generates a DC signal proportional to the beam current, which is integrated by our DAQ system.

The main requirement for $\mathrm{BCM}$ resolution is that it be much smaller than window-to-window beam intensity jitter. BCM resolution is measured by comparing simultaneous beam current measurements from both BCMs. The BCM resolution is 


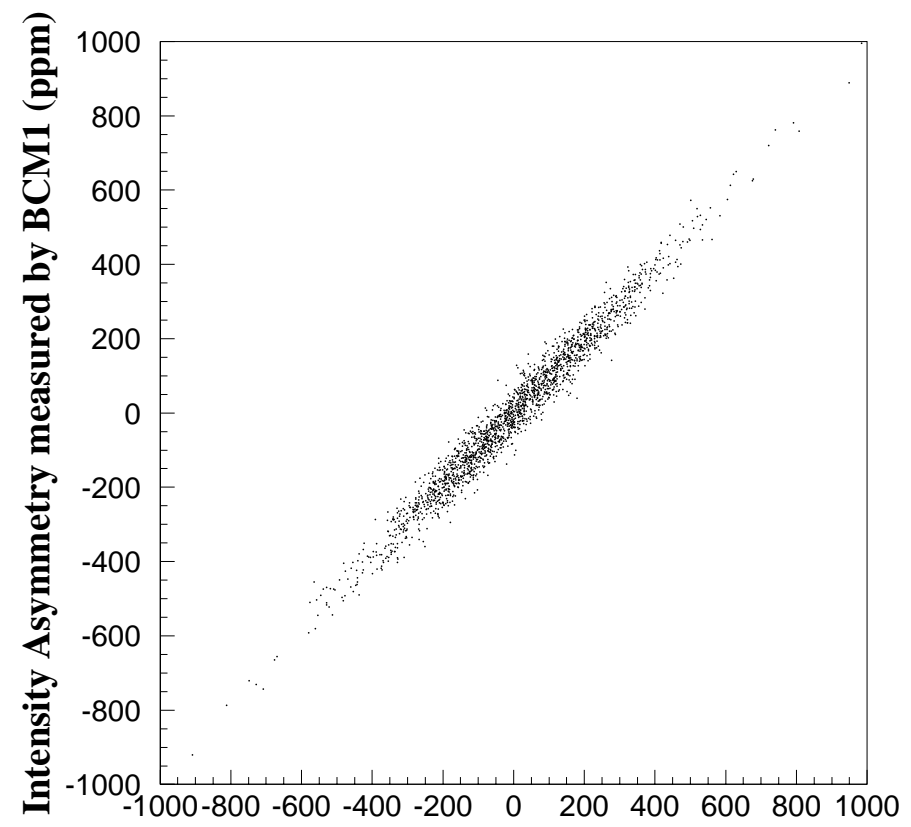

Intensity Asymmetry measured by BCM2 (ppm)

Figure 3.6: A correlation plot of the two beam intensity monitors. Windowto-window intensity jitter as measured by BCM1 is plotted along the vertical axis. Window-to-window intensity jitter as measured by BCM2 is plotted along the horizontal axis. The residuals of the correlation show the monitor resolution to be better than $40 \mathrm{ppm}$.

given by the residuals of the correlation between the two measurements. Figure 3.6 shows such a correlation plot. The BCM resolution is found to be better than $40 \mathrm{ppm}$ (parts per million) integrated over one 32 ms helicity window at a beam current of $100 \mu \mathrm{A}$. Thus BCM noise is negligible compared with the window-to-window beam intensity jitter of $250 \mathrm{ppm}$.

\subsection{Target and Raster}

For our target we used the $15 \mathrm{~cm}$ liquid hydrogen cell of the Hall A cryotarget assembly. The target cell is a cylindrical aluminum container approximately $15 \mathrm{~cm}$ 
long and $6 \mathrm{~cm}$ in diameter. It is oriented horizontally, concentric with the electron beam. Liquid hydrogen flows into the cell through a hole in the top and out through a hole in the bottom at a transverse flow rate of $1 \mathrm{~m} / \mathrm{s}$. The liquid hydrogen is recirculated through a heat exchanger which maintains it at a temperature of $19 \mathrm{~K}$ and a pressure of 26 psi. Under these conditions the density of the liquid hydrogen has been measured to be $0.072 \mathrm{~g} / \mathrm{cm}^{3}$ [19].

A $100 \mu \mathrm{A}$ electron beam deposits about $500 \mathrm{~W}$ into the target [20]. This heat must be dissipated in order to maintain the hydrogen in the liquid state. While the heat exchanger in the cryogenic loop is more than adequate to handle 500 Watts, local heating along the beam path presents a different problem. Since all this power is concentrated in a beam spot of less than $100 \mu \mathrm{m}$, the possibility of local boiling and bubble formation is a concern. In order to spread the heat load over a larger volume, the beam is swept over a small area of the target face using a device called a raster $[21]$.

The raster consists of two pairs of magnetic coils straddling the beamline, one for horizontal and one for vertical scanning of the electron beam. The coils are driven by currents with triangular waveforms of different frequency and adjustable amplitude whose values are chosen to provide even and rapid scanning of a rectangular area up to $2.5 \mathrm{~mm}$ by $2.5 \mathrm{~mm}$ on the target face.

\section{Target Boiling}

Target boiling can increase fluctuations in the normalized detector flux if the bubble formation occurs on a time scale comparable to the integration time of $32 \mathrm{~ms}$, resulting in a broadening of $\sigma\left(A_{\text {pair }}\right)$ beyond that due to counting statistics. Tests were conducted in October 1997, prior to the main production run, to gauge the effect of target boiling and determine an acceptable raster size. The beam energy 


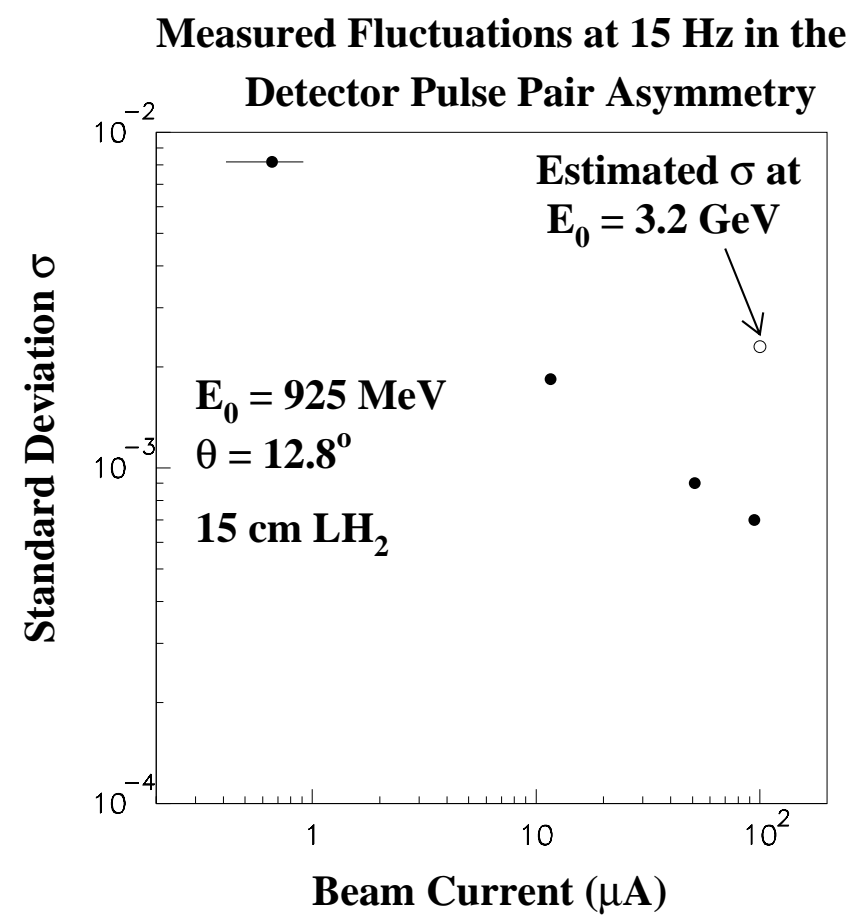

Figure 3.7: The fluctuations $\sigma\left(A_{\text {pair }}\right)$ in the normalized detector asymmetry are shown as a function of beam current on a log-log plot. This boiling test was carried out at lower beam energy (and therefore higher elastic scattering cross section) than the actual experiment.

was $925 \mathrm{MeV}$, resulting in a scattering rate in each detector of $\sim 50 \mathrm{MHz}$ for a beam current of $100 \mu \mathrm{A}$. The beam current was varied for several different raster sizes and we checked to see that the RMS detector fluctuations $\sigma$ varied with the beam intensity $I$ like $\sigma \propto I^{-1 / 2}$, as we would expect if the fluctuations are dominated by counting statistics of the scattering rate. The results of the test for a raster size of $2 \mathrm{~mm}$ are shown on a log-log plot in Figure 3.7. It can be seen that the dependence is approximately linear all the way to $100 \mu \mathrm{A}$, indicating that boiling is not appreciable at this raster size.

A closer look at the data reveals some evidence of boiling for smaller raster sizes at high beam current. Figure 3.8 shows several data points at different beam 


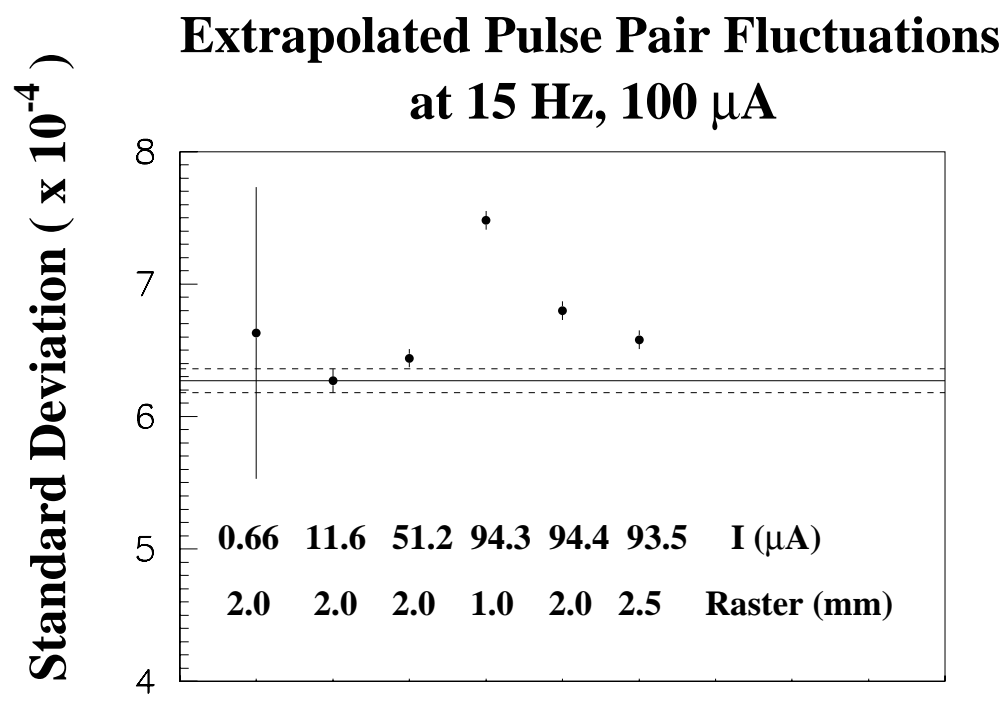

Figure 3.8: The fluctuations $\sigma\left(A_{\text {pair }}\right)$ in the normalized detector asymmetry are tested for statistical behavior by extrapolating each measured width to the corresponding width at a beam current of $100 \mu \mathrm{A}$. Evidence of additional noise is seen at the highest beam currents for smaller raster sizes.

currents and raster sizes, each extrapolated to show the expected detector widths at a beam current of $100 \mu \mathrm{A}$. Clear evidence of statistical broadening is seen at higher beam currents, and the broadening is seen to decrease with increasing raster size. From this data we estimate that the amount of boiling with a $2.5 \mathrm{~mm}$ raster is about $200 \mathrm{ppm}$. At the kinematics of our production run, the detector widths are about 3700 ppm, so we expect boiling to be negligible. Based on this test, we concluded that target boiling would not be a problem as long as we used a large raster size.

\subsection{Spectrometers}

We use both Hall A spectrometers, placed symmetrically at $\pm 12.3^{\circ}$ lab scattering angle, to select the elastically-scattered electrons. Normally these spectrometers are used in coincidence to detect both scattered particles from an electron-nucleon scattering event. For this reason one spectrometer is referred to as the "electron arm" 


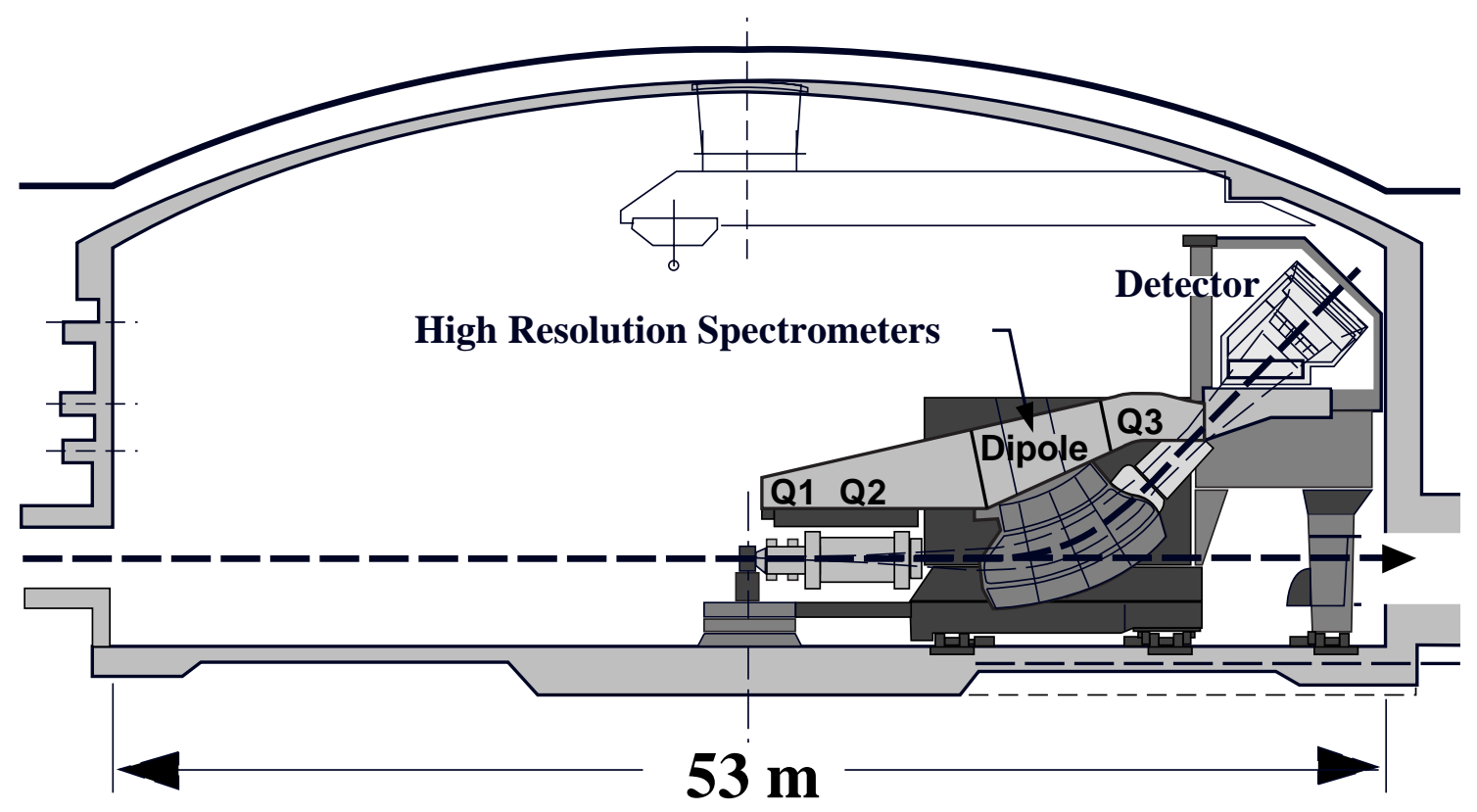

Figure 3.9: Hall A high-resolution spectrometer.

and the other as the "hadron arm." We detect only scattered electrons with both spectrometers, however. Using two spectrometers merely allows us to detect twice as many electrons and thereby increase the statistical precision of our measurement.

The two spectrometers are functionally identical. A diagram of a spectrometer is shown in Figure 3.9. The acceptance of each spectrometer is defined by a 5.5 millisteradian rectangular collimator. Each spectrometer consists of three quadrupoles and a dipole arranged in a QQDQ configuration. Electrons entering the spectrometer are bent by 45 degrees out of the scattering plane and are brought to a focus in a horizontal plane nearly $10 \mathrm{~m}$ above the beamline. The spectrometer detector package includes a vertical drift chamber at the focal plane which provides energy resolution of a few parts in $10^{4}$.

The spectrometers focus elastically-scattered electrons into a narrow stripe along the focal plane, as shown in Figure 3.10. We placed a long, narrow detector along the elastic stripe just above the focal plane of each spectrometer so that it inter- 


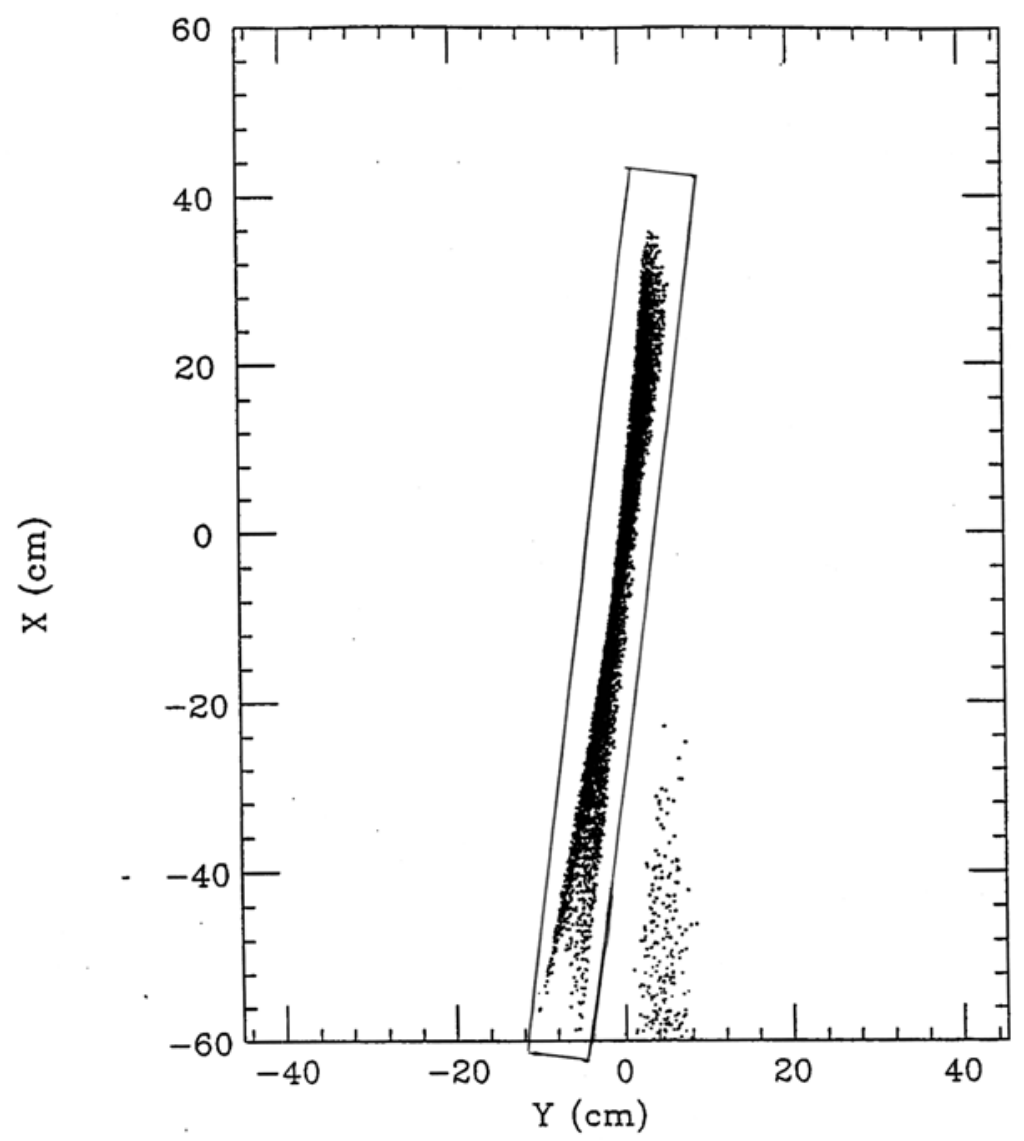

Figure 3.10: The position of scattered electrons at the spectrometer focal plane. The elastically scattered electrons are located in a narrow stripe. The outline of the HAPPEX detector is shown. The less dense band to the right are inelastic events due to pion production, which is the threshold for inelastic background.

cepted most elastic and no inelastic trajectories. The signals from these detectors were sent to the counting house where they were integrated and digitized by currentintegrating ADC boards. The vertical drift chambers were turned off during production running. They were used only in separate diagnostic runs to determine the average $Q^{2}$ at our kinematics and to verify that our detectors were properly aligned over the elastic stripe. 


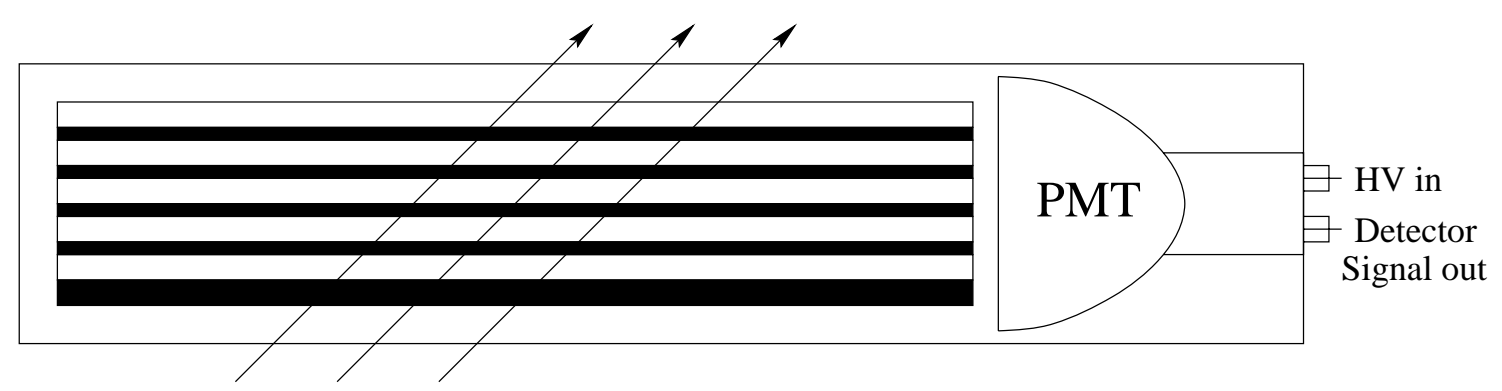

Direction of Scattered Electrons

Figure 3.11: HAPPEX detector.

\section{7 $\quad$ Detectors}

The clean separation of elastic from inelastic events afforded by the high-resolution spectrometers allowed us to place a total-absorption detector on the spectrometer focal plane over the elastic stripe and integrate the resulting signal. Although integration of the scattered flux reduces the complexity of the data acquisition system versus counting individual electrons, poor detector resolution for single electrons can add significantly to $\sigma\left(A_{\text {pair }}\right)$, the statistical width of the distribution of pair asymmetries. If $\Delta E / E$ is the fractional resolution for a single particle shower and $N_{s}$ is the total number of scattered electrons detected in a single window pair, then

$$
\sigma\left(A_{\text {pair }}\right)=\frac{1}{\sqrt{N_{s}}}\left[1+\left(\frac{\Delta E}{E}\right)^{2}\right]^{\frac{1}{2}}
$$

(compare with Equation 2.3).

The energy resolution of the detectors is optimized by using a layered sandwich design. Each detector consists of a 0.5 inch lead preradiator followed by five layers of 0.5 inch lucite alternated with 0.25 inch lead layers, as shown in Figure 3.11. The sandwich is $10 \mathrm{~cm}$ wide by $150 \mathrm{~cm}$ long. A Burle 8854 five-inch photomultiplier tube (PMT) is placed at one end of the lead-lucite sandwich to detect Cerenkov radiation as the electrons shower. GEANT simulations show that this design provides an 
energy resolution of $\sim 15 \%$ [20], which broadens the statistical width $\sigma\left(A_{\text {pair }}\right)$ by only about $1 \%$.

The resulting PMT output current signal is proportional to the electron scattering rate. This signal is sent to the HAPPEX DAQ system in the Hall A counting house where it is integrated by a current-integrating ADC channel. The PMT high voltage is adjusted to yield a PMT output signal current of $\sim 5 \mu \mathrm{A}$ at a maximum beam current of $100 \mu \mathrm{A}$, which results in an ADC output near full scale. Pedestal noise on the detector signal is typically less than $0.02 \%$ of full scale and causes negligible broadening of the statistical width of $\sigma\left(A_{\text {pair }}\right)$. The nonlinearity of the detectors was measured to be less than $1 \%$ by correlating detector response with beam current using data taken during the production run. This level of nonlinearity is sufficient for our experiment, as outlined in Section 2.2.3.

\subsection{Data Acquisition System and Integrating Elec- tronics}

The primary function of the HAPPEX data acquisition (DAQ) system is to integrate and digitize the signals from the detectors and beam monitors. All signals are integrated for $32 \mathrm{~ms}$ beginning $700 \mu$ s after the start of a helicity window. Waiting $700 \mu$ s allows the Pockels cell voltage and beam polarization to stabilize before making the cross-section measurement. The HAPPEX DAQ system was custom-built for the our experiment, and is completely separate from the standard Hall A DAQ system. Figure 3.12 shows a diagram of the HAPPEX DAQ system. A complete description of the system can be found in the Appendix. Here we concentrate on the integrating electronics. 


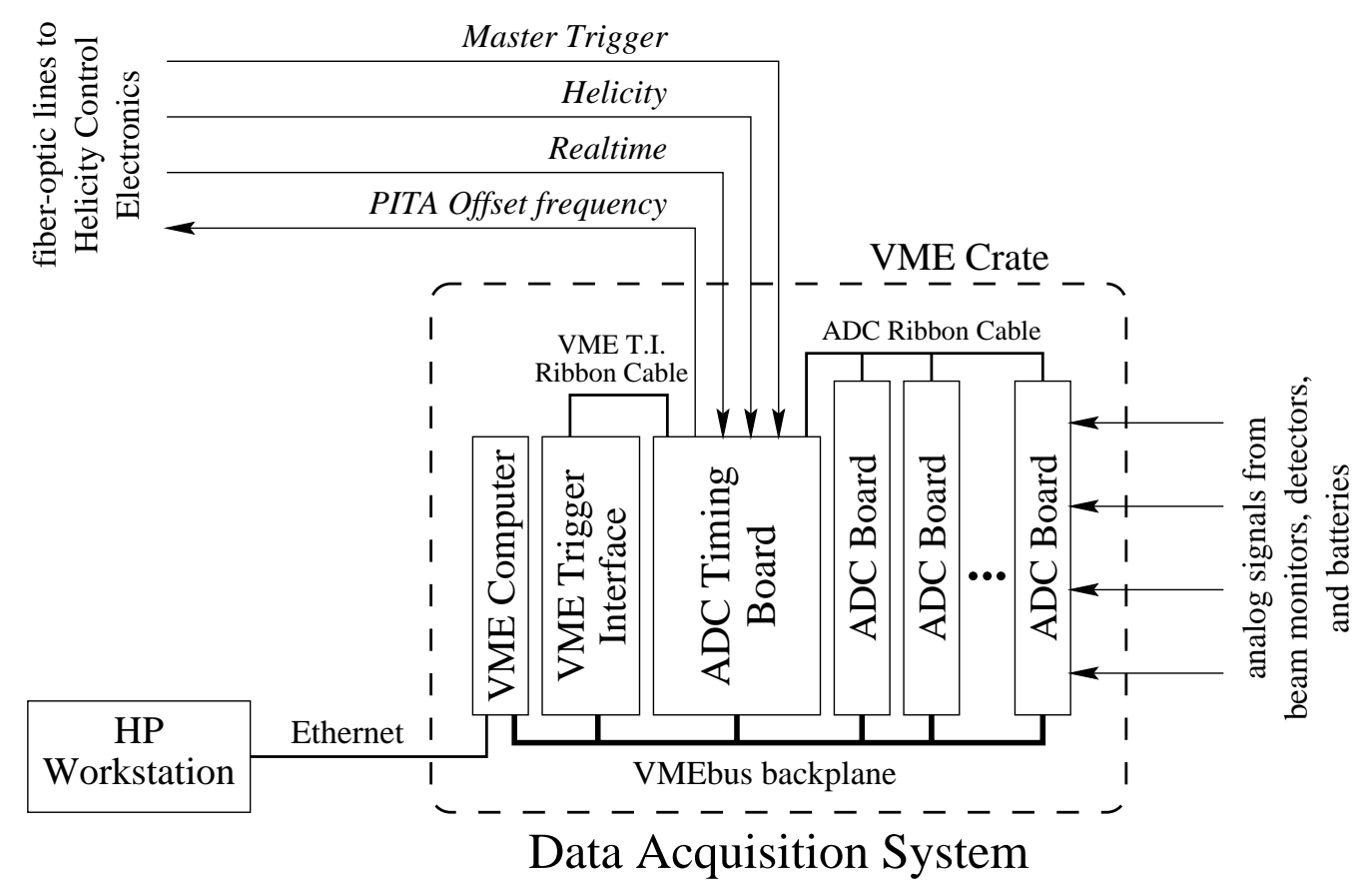

Figure 3.12: Data acquisition system.

\section{Integrating ADC Boards}

Integration and digitization are performed by custom-built 16-bit ADC boards designed for high resolution and low differential nonlinearity. These design goals are achieved using a single-slope sampling technique along with the use of pseudorandom noise injected onto the integrated signal before digitization [22]. Each board has four ADC channels which can be made voltage- or current-integrating with minor board modifications.

Each ADC channel consists of an input stage, an integrator with reset switch, two sample-and-hold circuits, a difference amplifier, a summing amplifier, and a 16bit ADC chip, as shown in Figure 3.13. For voltage-integrating channels, the input stage is a balanced-input differential amplifier followed by a resistor. It converts the input voltage signal to an appropriately-scaled current which is integrated onto the 


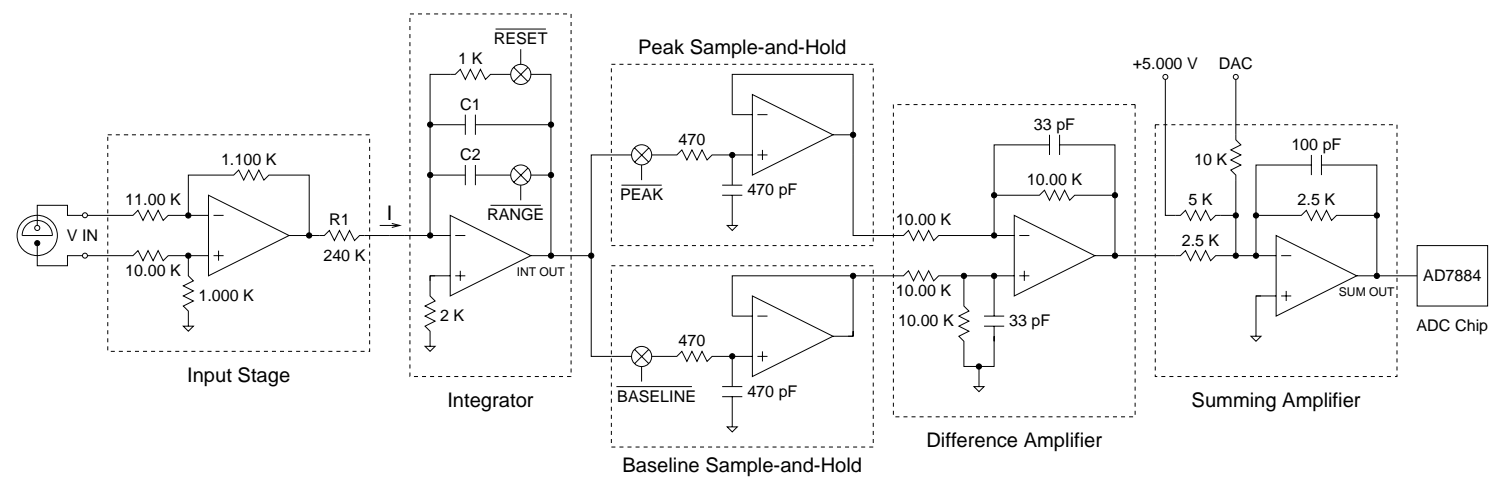

Figure 3.13: Circuit diagram of an ADC channel. A voltage-integrating channel is shown here.

feedback capacitor of an op-amp. For current-integrating channels, the differential amplifier is bypassed and the input current is integrated directly.

The integrator voltage is sampled and held once $700 \mu$ s after the beginning of the helicity window and again $32 \mathrm{~ms}$ later, near the end of the window. The difference between these two samples is the integrated result. A pseudorandom DAC voltage, referred to as "DAC noise," is added to the integrated result, and the sum is passed to a 16-bit ADC chip for digitization. The output of the 16-bit DAC is compressed to span 11 ADC bits. The ADC response to the DAC is calibrated in advance and the DAC contribution to the digitized result is subtracted during data analysis. The use of DAC noise reduces the differential nonlinearity of the ADC channel by averaging over $1700 \mathrm{ADC}$ bins in the vicinity of the integrated result.

A full discussion of the operation and performance of the ADC boards is included in the Appendix. Noise resolution of the ADC channel is 1.5 ADC bins, or $0.0025 \%$ of full scale, and does not increase when DAC noise is used. This level of measurement noise is completely negligible. The integral and differential nonlinearity of the ADC channel are less than $0.01 \%$. 


\subsection{Polarimetry}

We must know the electron beam polarization in order to extract the physics asymmetry from the measured raw asymmetry. The polarization $P_{e}$ of an ensemble of electrons is defined to be

$$
P_{e}=\frac{N_{+}-N_{-}}{N_{+}+N_{-}}
$$

where $N_{+}\left(N_{-}\right)$is the number of electrons with spin aligned parallel (antiparallel) to the axis of quantization. Under this definition of $P_{e}$ the physics asymmetry $A_{L R}$ is given by

$$
A_{L R}=\frac{A_{\text {raw }}}{P_{e}} .
$$

Both Mott and Møller scattering were used to measure the beam polarization. We will briefly describe the general theory of beam polarization measurements and then describe the Mott and Møller polarimeters used for our experiment.

Beam polarization can be measured using a known spin-dependent scattering process. In general, the cross section for a scattering process may be split into a spin-independent part $\sigma_{0}$ and a spin-dependent part $A \sigma_{0}$. The scattering cross sections for incident electrons of positive and negative helicity can then be written

$$
\begin{aligned}
& \sigma_{+}=\sigma_{0}(1+A), \\
& \sigma_{-}=\sigma_{0}(1-A) .
\end{aligned}
$$

Typically the beam polarization is reversible. Under the assumption that reversing the polarization interchanges $N_{+}$and $N_{-}$, the counting rates in a detector used to detect the scattered electrons are proportional to

$$
\begin{aligned}
& R_{+}=\sigma_{+} N_{+}+\sigma_{-} N_{-} \\
& R_{-}=\sigma_{+} N_{-}+\sigma_{-} N_{+} .
\end{aligned}
$$


where $R_{+}\left(R_{-}\right)$is the scattering rate for a beam of positive (negative) helicity.

Equations 3.1-3.4 may be combined to yield

$$
\frac{R_{+}-R_{-}}{R_{+}+R_{-}}=A P_{e} .
$$

Hence if $A$ is known, the beam polarization can be determined by measuring the scattering rates for each helicity. $A$ is called the analyzing power of the scattering process. In general, the analyzing power varies with incident beam energy and scattering angle. The detector is typically placed at the angle with maximum analyzing power for a given energy.

\subsubsection{Mott Polarimeter}

Mott polarimetry is based upon the electromagnetic scattering of polarized electrons from unpolarized heavy nuclei. The spin dependence of this interaction is the same spin-orbit effect responsible for the fine structure splitting in atomic electron transitions. The electron sees a magnetic field in its rest frame due to the relative motion of the charged nucleus. The interaction of the electron's spin with this magnetic field introduces a spin-dependent term in the interaction potential, given by [23]

$$
V=\frac{-Z e^{2}}{2 m^{2} c^{2} r^{3}} \mathbf{L} \cdot \mathbf{S} .
$$

Because of the $\mathbf{L} \cdot \mathbf{S}$ term in the scattering potential, Mott scattering is only sensitive to the component of the electron's spin which is transverse to the scattering plane. The analyzing power of Mott scattering can be calculated and is known as the Sherman function [24]. For $5 \mathrm{MeV}$ electrons scattered from gold nuclei, the maximum analyzing power occurs at a scattering angle of about $172^{\circ}$. Mott polarimetry is only practical at energies up to about $10 \mathrm{MeV}$. For higher energies 


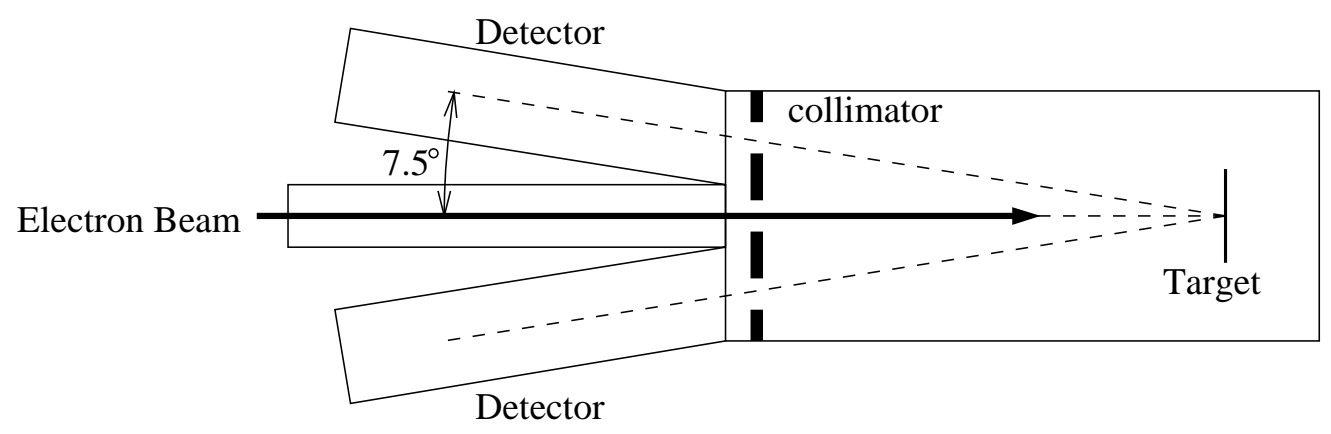

Figure 3.14: Diagram of the Mott polarimeter. Only two of the four detectors are shown.

the scattering angle for maximum analyzing power becomes impractically close to $180^{\circ}[25]$.

The Mott polarimeter $[26,27]$ is located in the $5 \mathrm{MeV}$ region of the injector. A diagram of the Mott polarimeter is shown in Figure 3.14. The polarized electron beam impinges on a gold foil and the scattered electrons are detected by four identical total energy detectors oriented symmetrically about the beamline at $172.5^{\circ}$ scattering angle. Collimators define the angular acceptance of the detectors and reduce background.

Two pairs of detectors oriented at $90^{\circ}$ with respect to one another are necessary to measure both components of transverse polarization. A Wien filter upstream of the Mott polarimeter is used to rotate the beam polarization vector so that it is perpendicular to the beam direction.

\subsubsection{Møller Polarimeter}

Møller polarimetry is based on the electromagnetic scattering of polarized beam electrons from polarized electrons in a magnetized target. The cross section for longitudinally-polarized electron-electron scattering is calculable in quantum electrodynamics. In the extreme relativistic limit, the cross section under one photon 

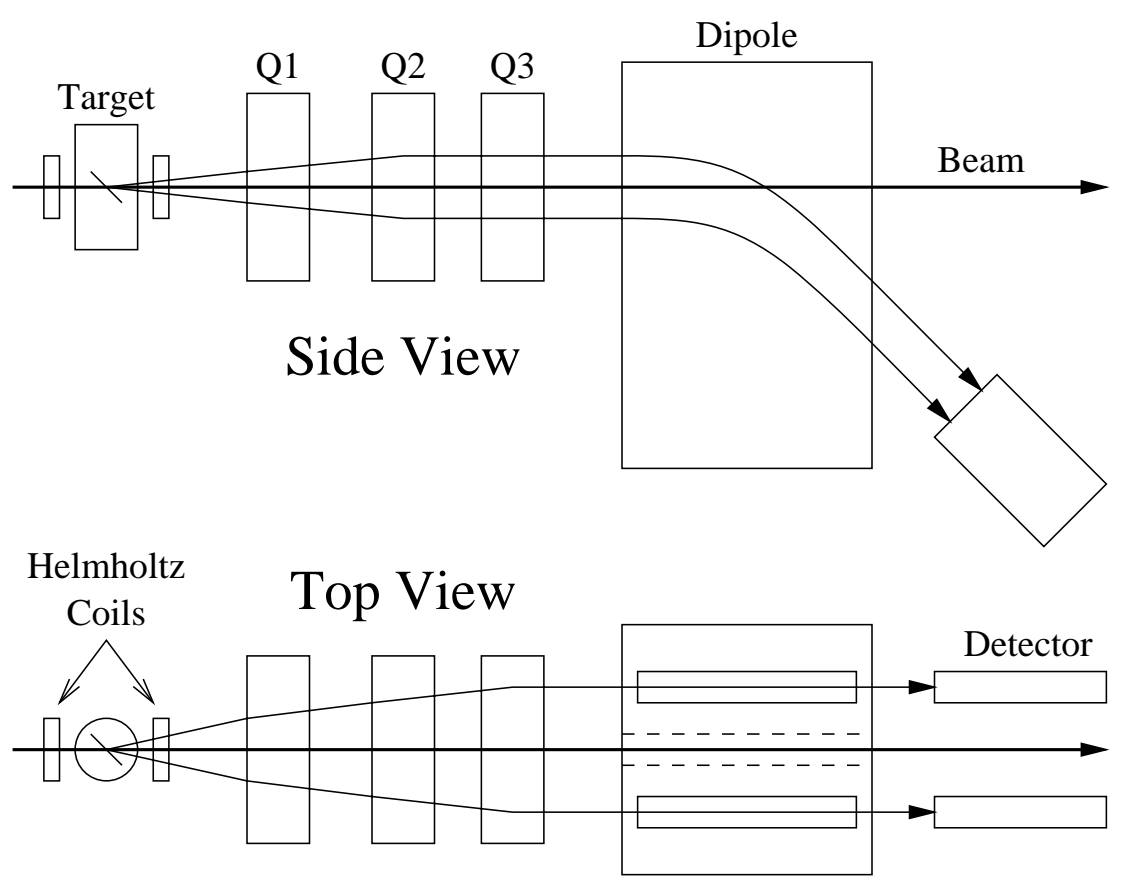

Figure 3.15: Diagram of the Møller polarimeter.

exchange is given by [28]

$$
\left.\frac{d \sigma}{d \Omega}\right|_{c m}=\frac{\alpha^{2}}{8 E^{2}} \frac{2\left(3+\cos ^{2} \theta\right)^{2}}{\sin ^{4} \theta}\left[1+h_{1} h_{2} \frac{\sin ^{2} \theta\left(7+\cos ^{2} \theta\right)}{\left(3+\cos ^{2} \theta\right)^{2}}\right],
$$

where $\theta$ is the scattering angle in the center-of-momentum frame and $h_{1}$ and $h_{2}$ are the helicities of the incident and target electrons. Equation 3.5 is already split into spin-dependent and spin-independent parts, so the analyzing power of Møller scattering can immediately be read off as

$$
A_{M \varnothing l l e r}=\frac{\sin ^{2} \theta\left(7+\cos ^{2} \theta\right)}{\left(3+\cos ^{2} \theta\right)^{2}} .
$$

The maximum analyzing power of $7 / 9$ occurs at a scattering angle of $90^{\circ}$ in the center of momentum frame, which corresponds to lab scattering angle of $3^{\circ}$ at a beam energy of $3.356 \mathrm{GeV}$.

The Møller polarimeter [29] is a permanent part of the Hall A beamline, located just upstream of the target. A diagram of the Møller polarimeter is shown in Fig- 
ure 3.15. The polarized electron beam impinges on a $14 \mu \mathrm{m}$ thick supermendur foil at an angle of $\pm 20^{\circ}$. The foil is magnetized by a pair of Helmholtz coils straddling the target chamber which provide a field of $28 \mathrm{mT}$ either parallel or antiparallel to the beam direction, yielding an effective target polarization of $7.6 \%$.

Due to the small scattering angle, the scattered electrons must be carefully separated from the main beam before they can be detected. This is achieved by three quadrupole magnets which direct the scattered electrons over the two collimating slots while the main beam passes undeflected along the quadrupole axis. Then the scattered electrons are bent downward by a large dipole magnet and pass through the two collimating slots which reduce Mott background by selecting electrons of the correct energy. Both Møller electrons are detected in coincidence. The error in the Møller measurement is dominated by the systematic error in the measurement of the target polarization, estimated to be less than $5 \%$ relative.

The beam polarization was measured daily throughout the production run using both polarimeters. The average of all measurements was taken as the measured result. 


\section{Chapter 4}

\section{Analysis and Results}

The production run took place between April 17 and May 18, 1998. Here we present the results of the data analysis, representing 78 Coulombs of electrons on target.

\subsection{Raw Asymmetries}

The raw asymmetry is calculated for each window pair for each detector $i=1,2$ :

$$
A_{\text {pair }, i}=\frac{\operatorname{det} i_{R} / \mathrm{bcm}_{R}-\operatorname{det} i_{L} / \mathrm{bcm}}{\operatorname{det} i_{R} / \mathrm{bcm}_{R}+\operatorname{det} i_{L} / \mathrm{bcm}_{L}},
$$

where det1 (det2) is the digitized integrated signal from the detector in the electronarm (hadron-arm) spectrometer and $\mathrm{bcm}$ is the digitized integrated signal from the beam current monitor. Each pair asymmetry is weighted by the integrated beam current when computing the average:

$$
\left\langle A_{\text {pair, }, i}\right\rangle=\frac{\sum_{\text {pairs }}\left(\mathrm{bcm}_{R}+\mathrm{bcm}_{L}\right) A_{\text {pair }, i}}{\sum_{\text {pairs }}\left(\mathrm{bcm}_{R}+\mathrm{bcm}_{L}\right)} .
$$

The production run was divided into about 400 data runs, each up to an hour long. We calculate an average raw asymmetry for each detector for each data run using the prescription above, with an error given by the standard error in the mean. Then the average for all runs, weighted by the error for each run, is computed to 


\section{Electron-Arm Detector Asymmetry by Run}

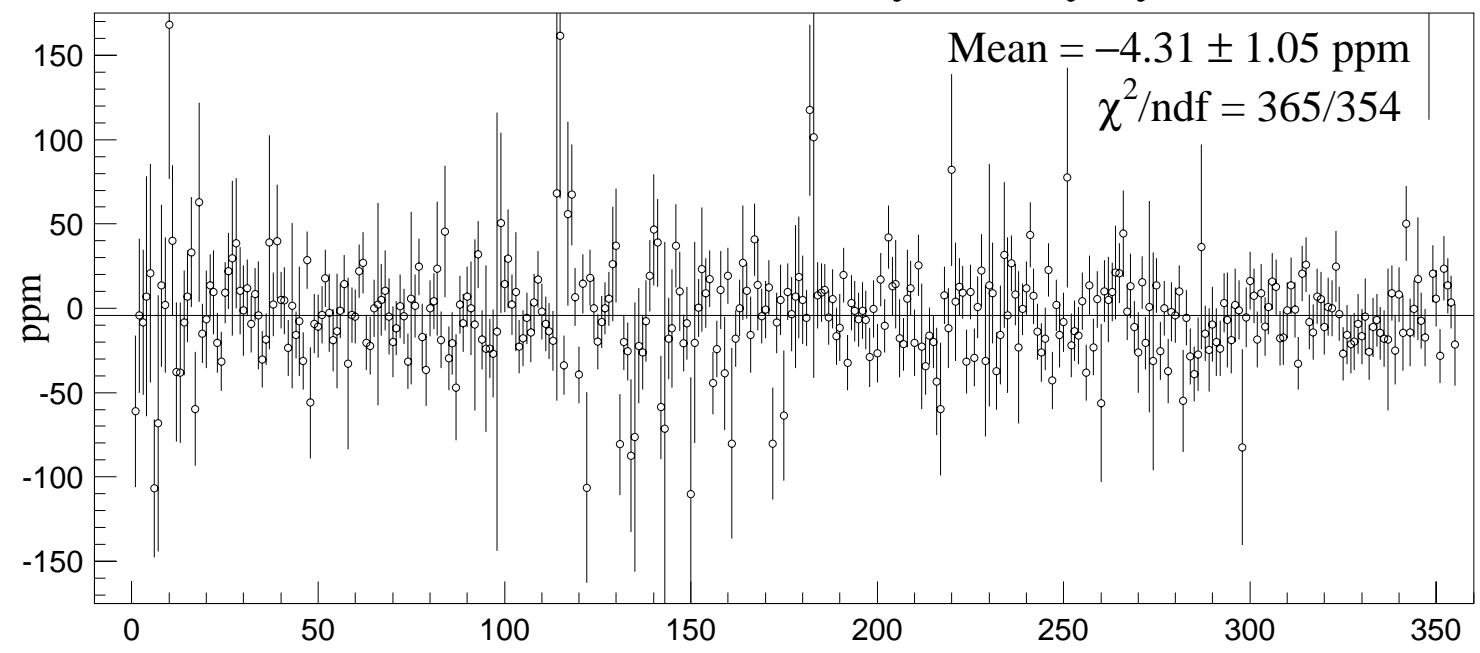

Hadron-Arm Detector Asymmetry by Run

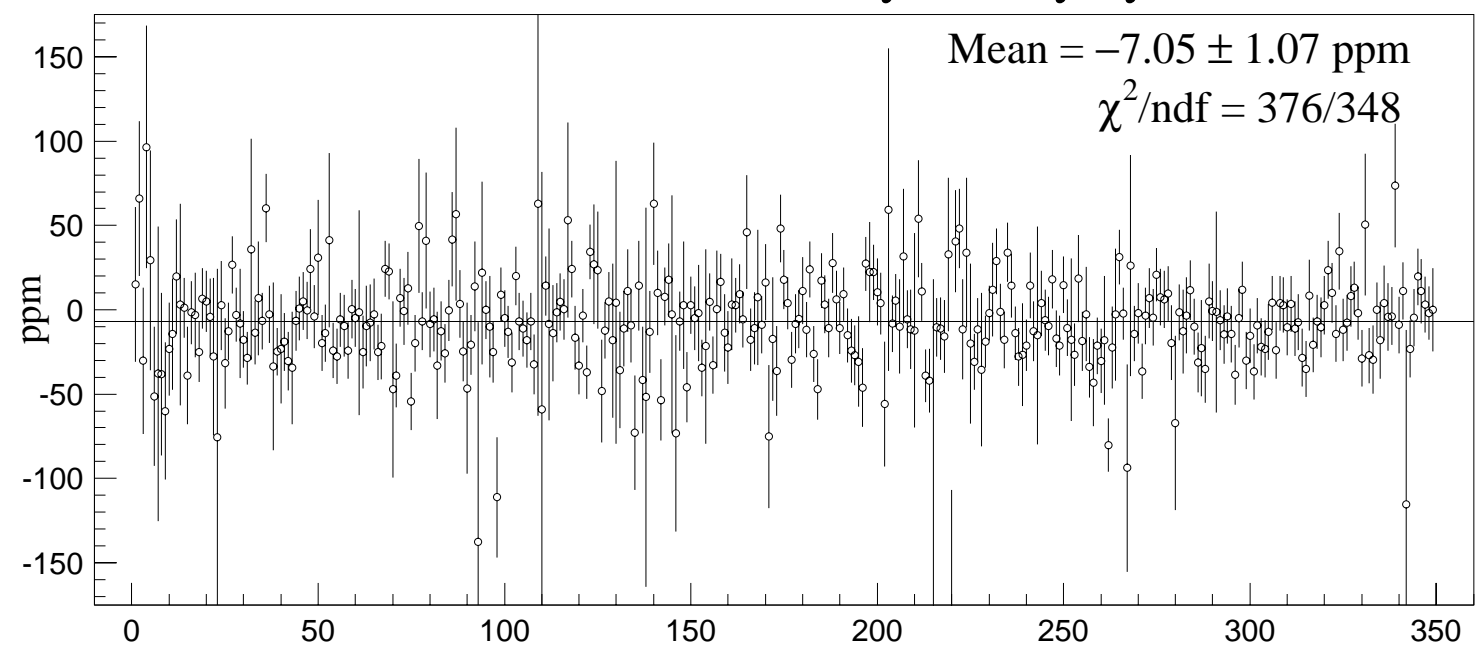

Figure 4.1: A plot of the raw asymmetries measured by each detector for each data run.

yield the final raw asymmetry measured by each detector. Figure 4.1 shows the asymmetries and errors for each run along with the average raw asymmetry for each detector.

In order to avoid biasing the experimental result, we made as few cuts as possible on the experimental data. Of all the data runs taken, we only cut those runs or 


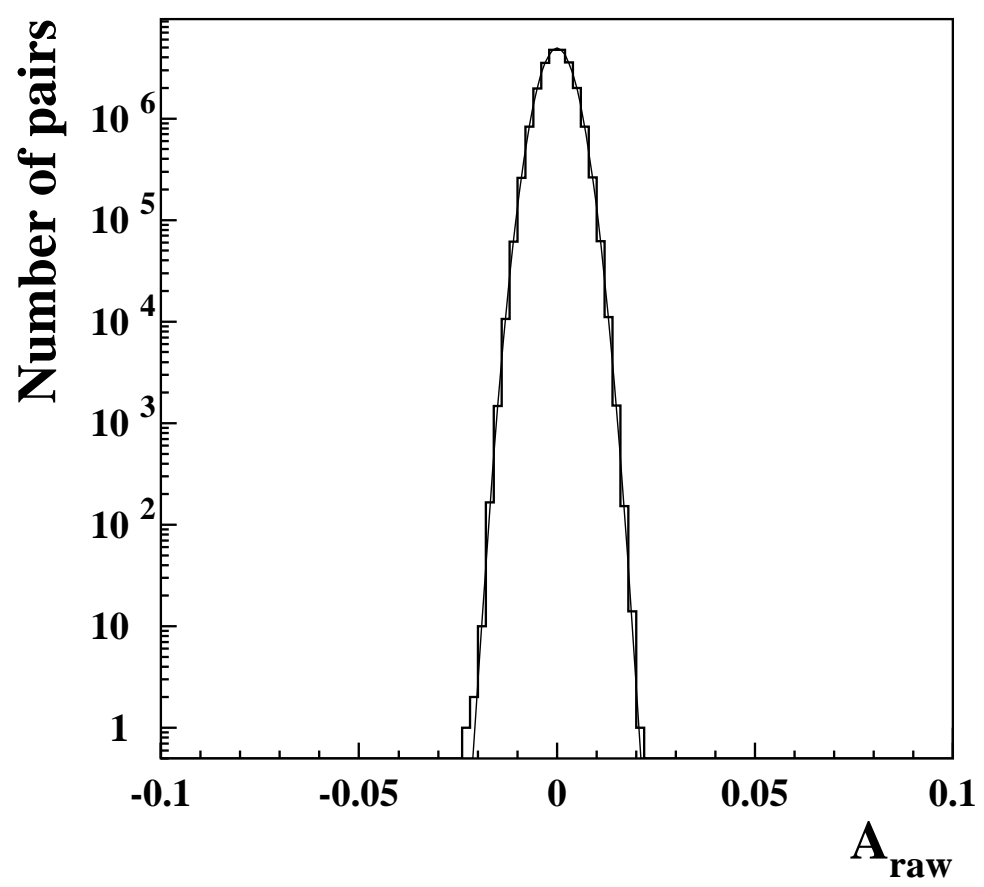

Figure 4.2: A histogram of raw pair asymmetries. All data for beam current greater than $80 \mu \mathrm{A}$ is shown, representing $\sim 95 \%$ of the data sample. The line is a Gaussian fit with $\sigma=3800 \mathrm{ppm}$.

parts of runs for which the beam current was less than $3 \mu \mathrm{A}$ or when experimental equipment such as the spectrometers or target was clearly malfunctioning. In particular, we did not make any cuts on any helicity-correlated quantity, including position and intensity monitor differences or detector asymmetries.

A histogram of the distribution of raw pair asymmetries is shown in Figure 4.2. The distribution is Gaussian over seven orders of magnitude. The half-width of the distribution is equal to $\sigma\left(A_{\text {pair }}\right)$, as discussed in Section 2.2.2. We find that $\sigma\left(A_{\text {pair }}\right)$ is $\sim 3800$ ppm (parts per million). 


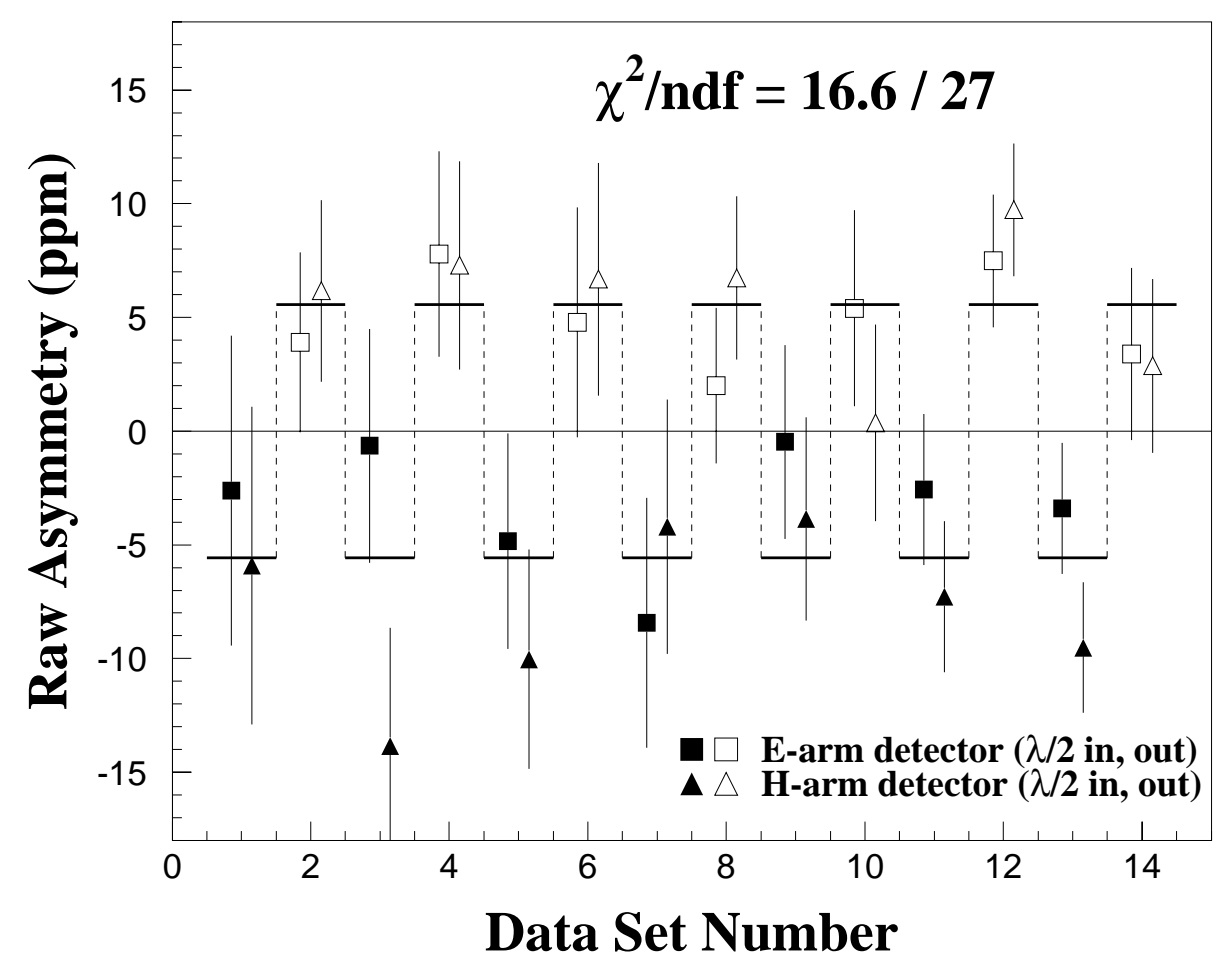

Figure 4.3: A plot of the average raw asymmetry measured by each detector for each $\lambda / 2$ data set. The $\lambda / 2$ plate is inserted for the odd-numbered data sets. A clear correlation is seen between the sign of the asymmetry and the presence of the $\lambda / 2$ plate.

\subsection{Passive Helicity Reversal}

An important test for the presence of false asymmetries is through the insertion of a half-wave $(\lambda / 2)$ plate in the source laser beam, as discussed in Section 3.2. This reverses the correlation between the electronic helicity signals and the actual helicity of the electron beam. If the analysis software does not make allowances for the insertion of the $\lambda / 2$ plate, it will calculate the opposite sign for the asymmetry. The position of the $\lambda / 2$ plate was toggled every 24-48 hours throughout the production run, so that about half the data was taken with it inserted. The production run was thereby divided into 14 data sets, with the $\lambda / 2$ plate inserted for the odd sets. 
The raw asymmetries calculated by the analysis software for each data set are shown in Figure 4.3. A clear correlation between the presence of the half-wave plate and the sign of the asymmetry is seen. This is a powerful demonstration that the measured asymmetry is due to parity violation rather than a spurious electronic effect. Table 4.1 shows the overall average raw asymmetries for each detector for each half-wave plate setting. The sign of the $\lambda / 2$ out data is corrected when combining the data sets.

\begin{tabular}{|l|ll|r|}
\hline & $\lambda / 2$ out & $\lambda / 2$ in & Combined \\
\hline det1 & $5.1 \pm 1.4$ & $-3.3 \pm 1.6$ & $-4.31 \pm 1.05$ \\
det2 & $6.2 \pm 1.5$ & $-8.1 \pm 1.6$ & $-7.05 \pm 1.07$ \\
\hline Total & $5.6 \pm 1.0$ & $-5.7 \pm 1.1$ & $-5.64 \pm 0.75$ \\
\hline
\end{tabular}

Table 4.1: Average raw asymmetries (in ppm).

\subsection{Performance of Intensity Feedback System}

Helicity-correlated beam intensity differences were reduced using the intensity feedback system described in Section 2.3.2. Each data run was divided into 3-minute miniruns. The feedback system adjusted the Pockels cell voltage offset at the end of each minirun in order to null the measured intensity asymmetry.

Figure 4.4 gives a dramatic demonstration of the effectiveness of the feedback system. The top plot shows the average intensity asymmetry during each minirun of a one hour data run. The Pockels cell voltage offset was accidentally set to zero (rather than to the approximate null point) for the first minirun, causing a large intensity asymmetry. But the system feeds back at the end of the first minirun and immediately corrects for the measured intensity asymmetry, keeping it near zero for the remainder of the run. 


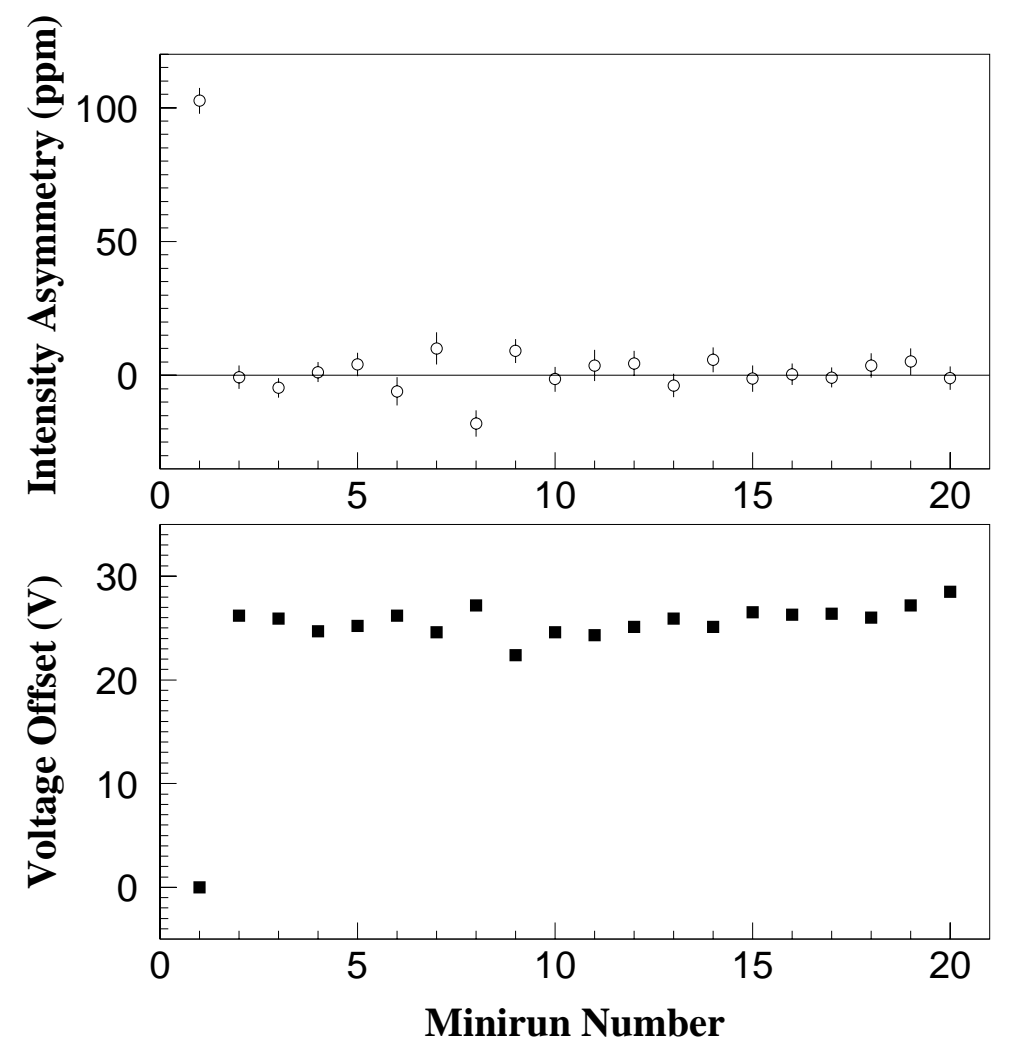

Figure 4.4: The top plot shows the intensity asymmetry during each minirun of Run 7817. The bottom plot shows the Pockels cell voltage offset for each minirun. The Pockels cell voltage offset was accidentally set to zero for the first minirun, causing a large intensity asymmetry.

Figure 4.5 shows the average intensity asymmetry for each data run. For the most part, the asymmetry is kept near zero. Large values are typically due to large first minirun asymmetries, as in the example above, or short data runs in which a large temporary intensity asymmetry can dominate the average. Since we do not apply cuts based on the intensity asymmetry, these runs are included in the data set. The average intensity asymmetry over the entire data set is $0.2 \mathrm{ppm}$, well below the $6 \mathrm{ppm}$ limit set in Section 2.2.3.

Figure 4.6 demonstrates the $1 / N$ convergence of the feedback scheme described 


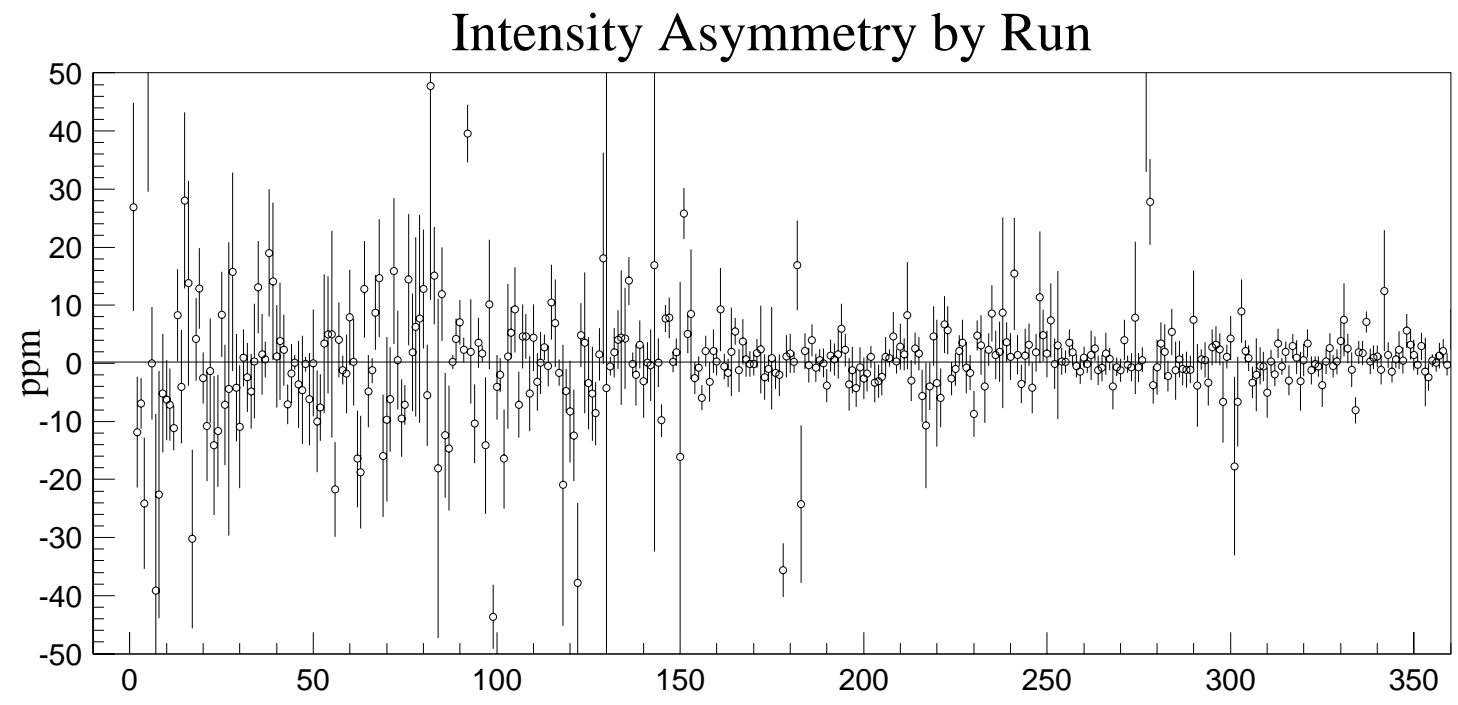

Figure 4.5: A plot of the average intensity asymmetry for each data run. The overall average intensity asymmetry is $0.2 \mathrm{ppm}$.

in Section 2.3.2. The average cumulative asymmetries are clearly more consistent with the $\sigma / N$ line than the $\sigma / \sqrt{N}$ line.

The feedback scheme converges as long as the PITA slope is correct within a factor of two. Figure 4.7 shows the divergence of the feedback scheme when the slope is wrong by more than a factor of two. The feedback scheme is designed to correct each minirun asymmetry back to zero. If the PITA slope used in calculating the correction is more than a factor of two smaller than the actual PITA slope, the system overshoots each correction and amplifies the asymmetry on the other side of zero. The intensity asymmetries for each minirun alternate sign and diverge exponentially until the limit of the voltage offset is reached.

This example of feedback divergence provides an opportunity to study beam loading. The accelerator turns intensity differences into energy differences because it is slightly resistive. This effect is known as beam loading. Figure 4.8 shows the correlation between the position difference measured by BPM12x and the intensity 


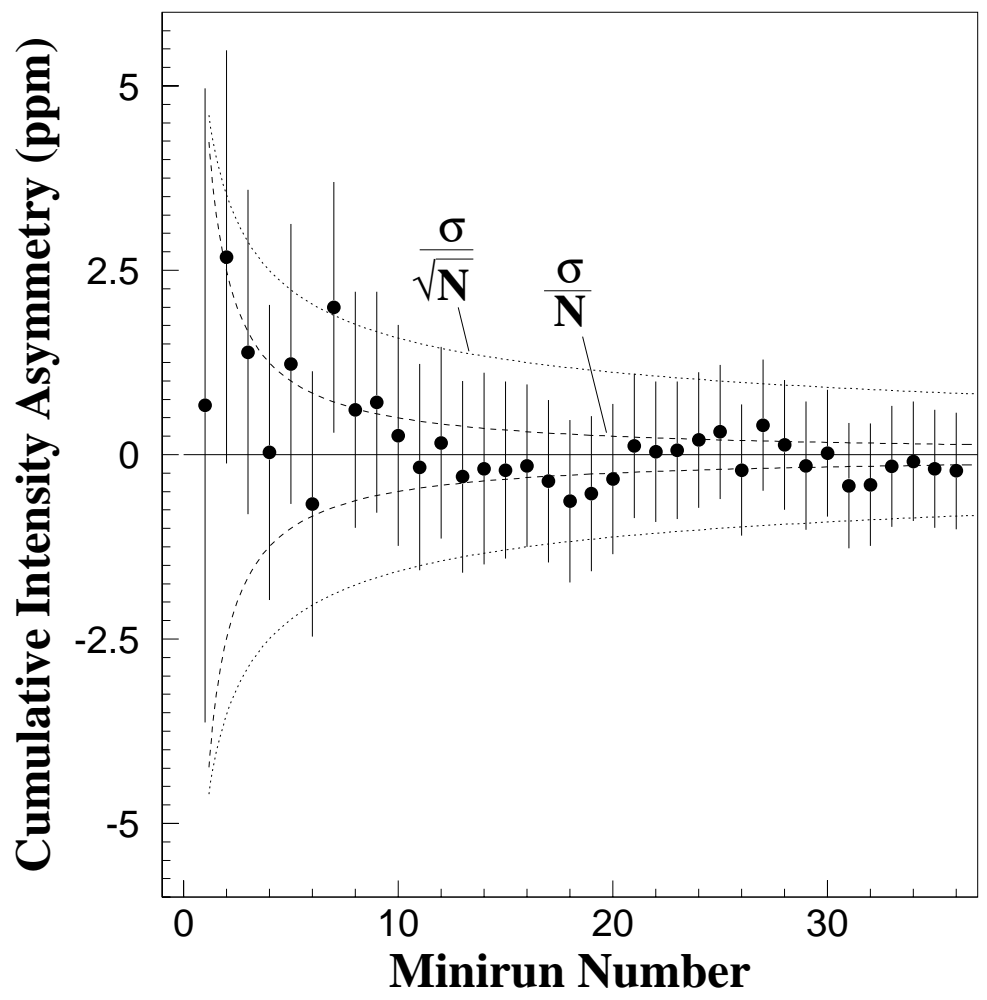

Figure 4.6: A demonstration of $1 / N$ convergence of the feedback scheme. The cumulative intensity asymmetry is plotted after each minirun of a feedback sequence. The cumulative intensity asymmetries are clearly more consistent with the $\sigma / N$ line.

asymmetry from the series of miniruns in Figure 4.7. We interpret this correlation to be due to beam loading. The size of the effect is $2 \mathrm{~nm} / \mathrm{ppm}$. The beam modulation analysis in Section 4.4 shows that the sensitivity of the measured asymmetry to the helicity-correlated energy difference is on the order of $0.5 \mathrm{ppm} / \mu \mathrm{m}$. Therefore we conclude that the systematic effect due to beam loading is negligible for intensity asymmetries less than 100 ppm. 


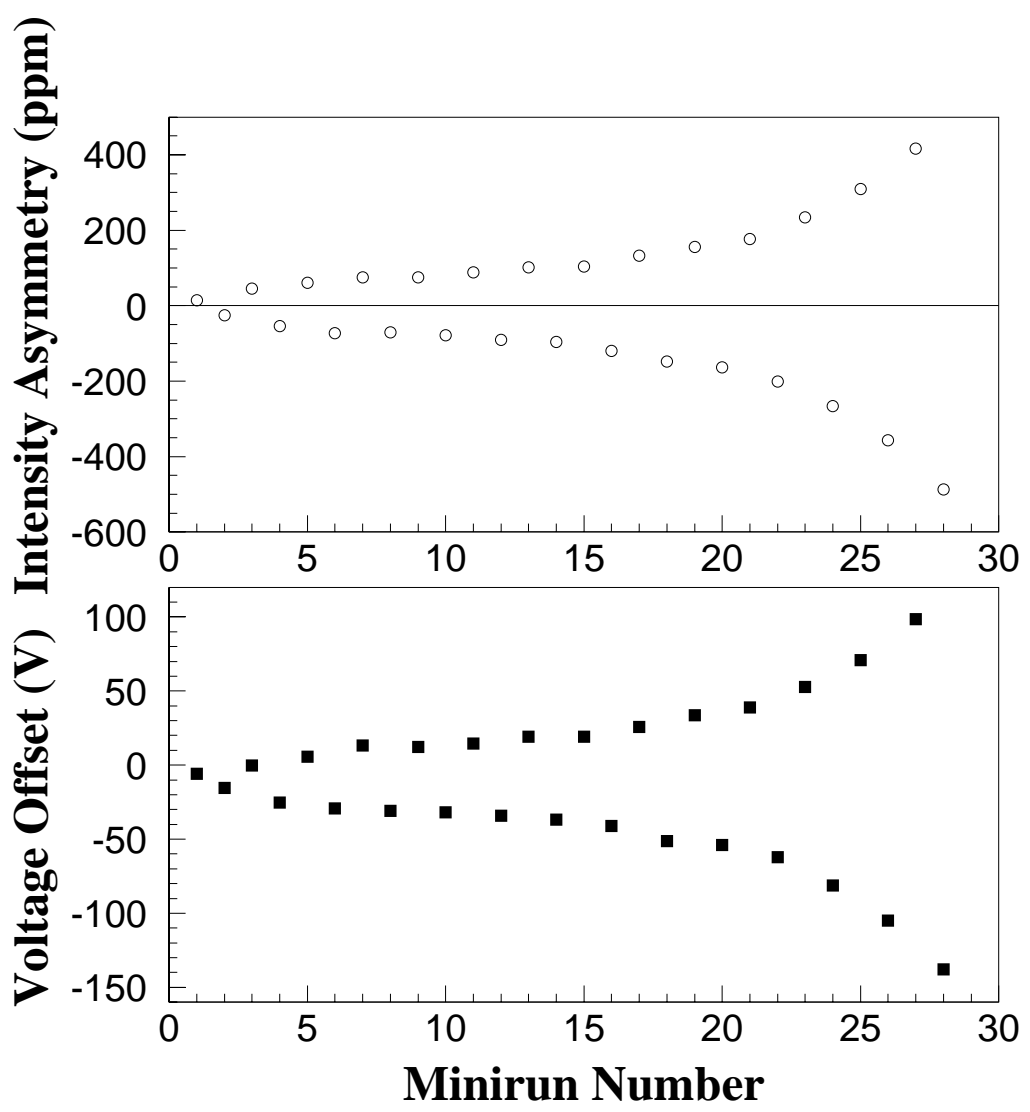

Figure 4.7: The PITA slope used for this feedback sequence is more than a factor of 2 too small. The feedback scheme diverges exponentially.

\subsection{Beam Modulation Analysis}

The false component of the raw asymmetry due to helicity-correlated differences in beam position, angle, and energy is determined by a beam modulation scheme described in Section 2.3.3. In this section we outline the beam modulation analysis and present the results. A complete discussion of the analysis can be found in References [30] and [20].

We assume that the normalized flux $\sigma_{i}$ measured by each detector $(i=1,2)$ depends on the five beam parameters $M_{1}, \ldots, M_{5}$, which represent the beam position measured by BPM3Ax, BPM3Ay, BPM3Bx, BPM3By, and BPM12x. By ramping the seven 


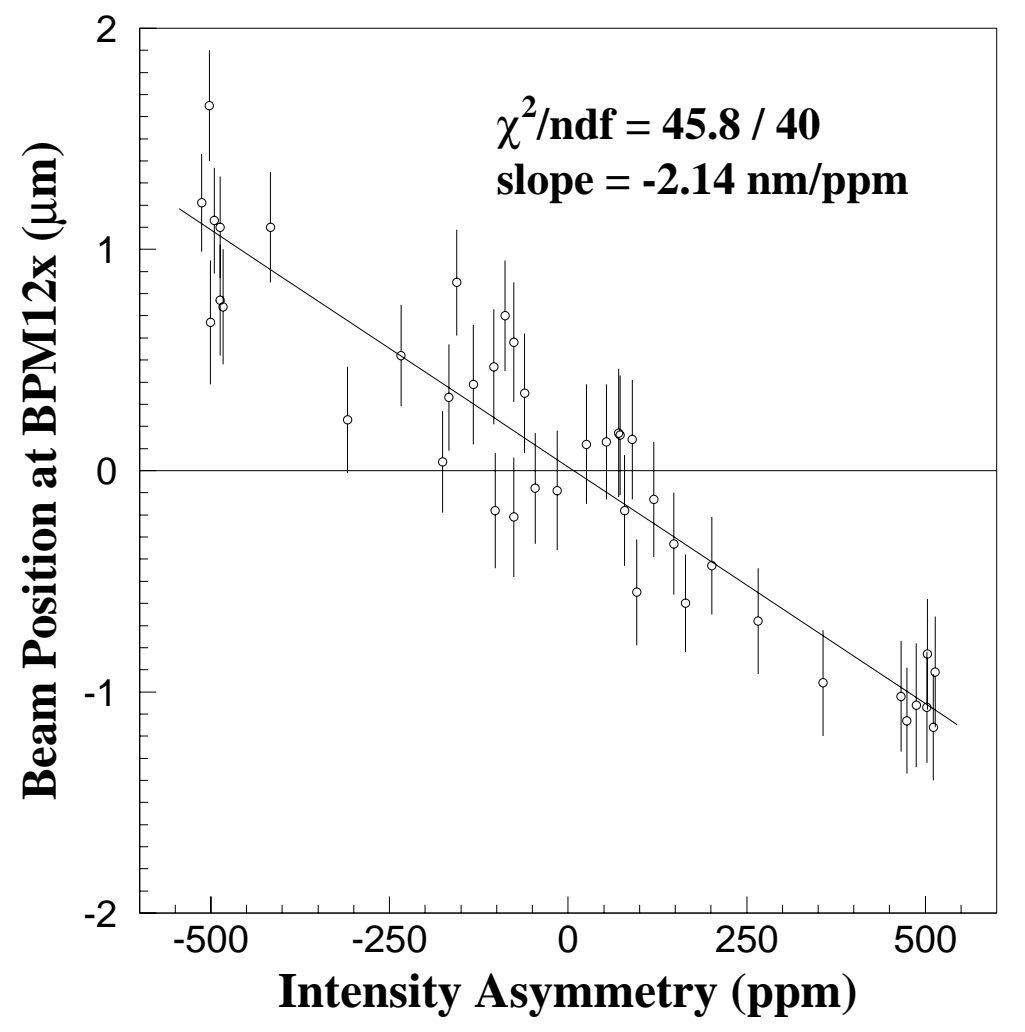

Figure 4.8: A plot showing the effects of beam loading. The horizontal beam position as measured by the energy BPM is plotted on the y-axis, and the intensity asymmetry is plotted on the $\mathrm{x}$-axis. Intensity differences are seen to induce energy differences of $2 \mathrm{~nm} / \mathrm{ppm}$.

steering coils $C_{1}, \ldots, C_{7}$ and energy vernier $C_{8}$, we measure the first-order responses $\partial M_{k} / \partial C_{j}$ and $\partial \sigma_{i} / \partial C_{j}$ of the beam position at monitor $M_{k}$ and the normalized detector flux $\sigma_{i}$, respectively, to the coil $C_{j}$. We are trying to find the sensitivity $\partial \sigma_{i} / \partial M_{k}$ of the normalized detector flux to the beam position at each monitor. The responses and sensitivities are related by the matrix equation

$$
\frac{\partial \sigma_{i}}{\partial C_{j}}=\sum_{k=1}^{5} \frac{\partial \sigma_{i}}{\partial M_{k}} \frac{\partial M_{k}}{\partial C_{j}} .
$$

We solve this equation for $\partial \sigma_{i} / \partial M_{k}$ by inverting the matrix $\partial M_{k} / \partial C_{j}$. Thus an important requirement of the beam modulation scheme is that the coils perturb the 


\begin{tabular}{|c|c|c|}
\hline $\begin{array}{c}\text { Monitor } \\
M_{j}\end{array}$ & $\begin{array}{l}\text { Detector } 1 \text { Sensitivity } \\
\left(\partial \sigma_{1} / \partial M_{j}\right) / 2 \sigma\end{array}$ & $\begin{array}{l}\text { Detector } 2 \text { Sensitivity } \\
\left(\partial \sigma_{2} / \partial M_{j}\right) / 2 \sigma\end{array}$ \\
\hline BPM3Ax & $-5.0 \pm 0.7 \mathrm{ppm} / \mu \mathrm{m}$ & $3.5 \pm 0.6 \mathrm{ppm} / \mu \mathrm{m}$ \\
\hline BPM3Ay & -0.5 & 0.05 \\
\hline BPM3Bx & 10.4 & -4.8 \\
\hline BPM3Вy & 0.03 & 0.03 \\
\hline BPM12x & $-0.2 \pm$ & 0.05 \\
\hline
\end{tabular}

Table 4.2: Sensitivities of normalized detector flux to changes in beam parameters.

position and angle of the beam independently, so that this matrix is sufficiently nonsingular. The measured sensitivities $\partial \sigma_{i} / \partial M_{k}$ are given in Table 4.2.

We also keep track of the right-left position differences $\Delta M_{k}^{L R}$. The false component of the measured raw asymmetry due to these position differences can be computed as

$$
A_{\text {false }}=\frac{1}{2 \sigma} \sum_{k=1}^{5} \frac{\partial \sigma_{i}}{\partial M_{k}} \Delta M_{k}^{L R},
$$

where $\sigma=\left(\sigma_{R}+\sigma_{L}\right) / 2$ is the average normalized detector flux. We compute $A_{\text {false }}$ for each window pair and take the average of all pairs weighted by the integrated beam current for each pair to determine the total false component.

The total false component of the raw asymmetry is found to be

$$
A_{\text {false }}=0.05 \pm 0.02 \mathrm{ppm} \text {. }
$$

Although the false asymmetry is statistically nonzero, it is less than $0.1 \mathrm{ppm}$ and thus negligible. Therefore no correction was applied to the measured asymmetry. The false asymmetry is small because helicity-correlated position differences are small, as shown in Figure 4.9. The average differences are all statistically consistent with zero. 

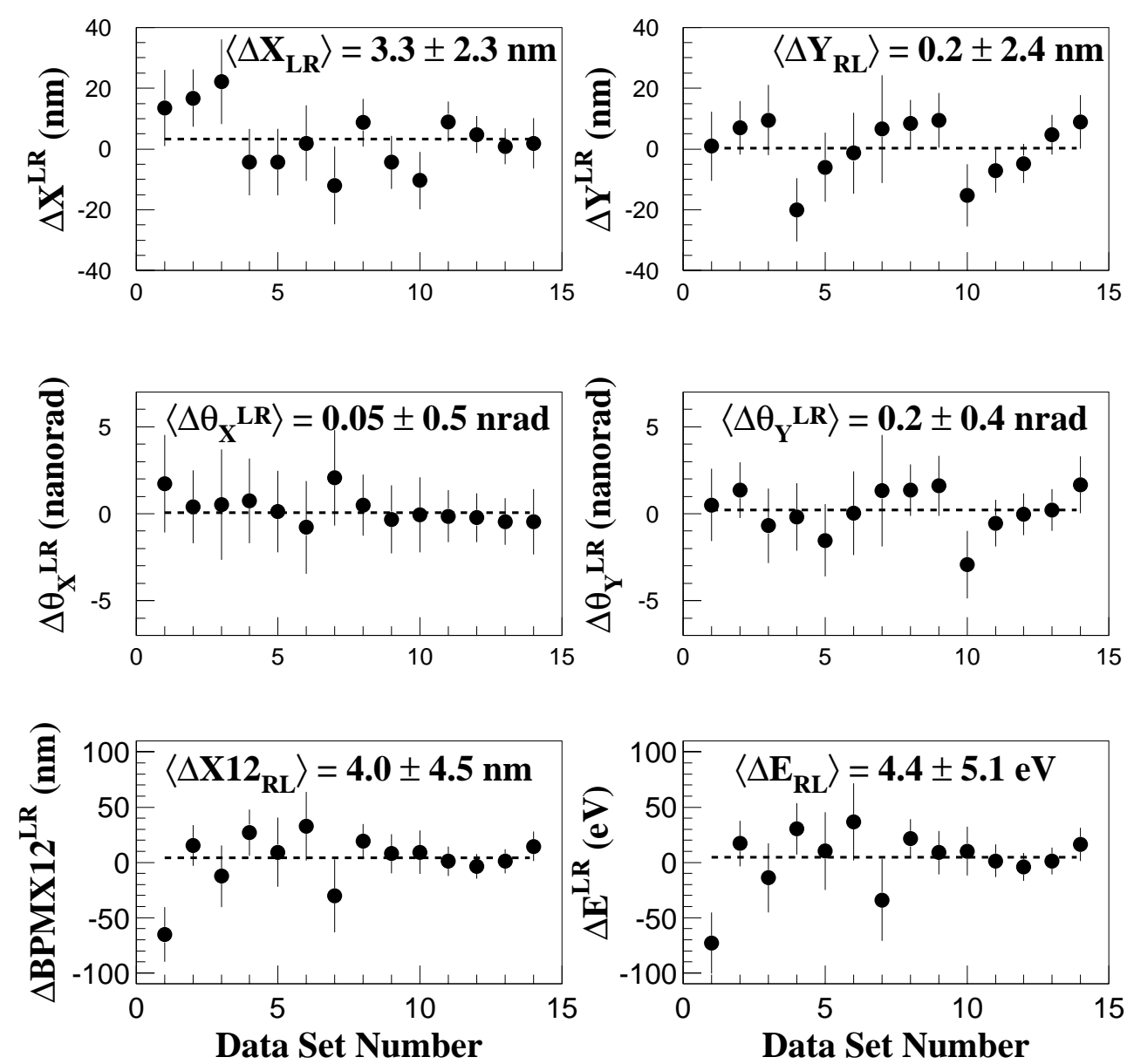

Figure 4.9: Plots of helicity-correlated beam differences. The top four plots show average beam position and angle differences at the target for each $\lambda / 2$ data set, extrapolated from measurements at BPM3A and BPM3B. The bottom two plots show energy differences as measured by BPM12x. The average differences are all statistically consistent with zero.

\subsection{Auxiliary Data Runs}

The standard Hall A data acquisition system was used to track individual scattering events for a few auxiliary runs described in this section. 


\subsubsection{Determination of $\left\langle Q^{2}\right\rangle$}

Since the target is an extended structure and the spectrometer has a finite acceptance, the HAPPEX detector will intercept scattering events which have a broad range of $Q^{2}$ values. To determine the average $Q^{2}$ of the events seen by our detector, we used the vertical drift chamber (VDC) to track individual events, using the HAPPEX detector as a trigger in a separate auxiliary run.

The four-momentum transfer squared of a scattering event is $Q^{2}=2 E E^{\prime}(1-$ $\cos \theta)$. The incident energy $E$ was $3.356 \mathrm{GeV}$ and was corrected slightly to compensate for energy lost in the target before scattering. The energy $E^{\prime}$ of the scattered electron and the scattering angle $\theta$ were determined from the VDC data. For elastic scattering, $E, E^{\prime}$, and $\theta$ are not independent, which provides a consistency check on the data. The average $Q^{2}$ for our experiment was determined to be $0.479 \pm 0.003(\mathrm{GeV} / \mathrm{c})^{2}$. Data from a typical $Q^{2}$ run is shown in Figure 4.10.

\subsubsection{Background Estimation}

Because we integrate the detector signal instead of counting individual events, there is no way to reject background events intercepted by our detector. Helicitycorrelated background due to inelastic scattering at the $\Delta$-resonance is therefore of concern. To estimate the resulting systematic error, we must first determine the sensitivity of our detectors to this source of background.

We performed a test designed to estimate the fraction of the integrated detector signal due to background events. The electron-arm spectrometer's magnetic fields were slowly increased in discrete steps to $20 \%$ above their normal values, steering the elastic stripe off the HAPPEX detector and into the spectrometer wall. Meanwhile, the hadron-arm fields were left at their normal values so that the elastic stripe 


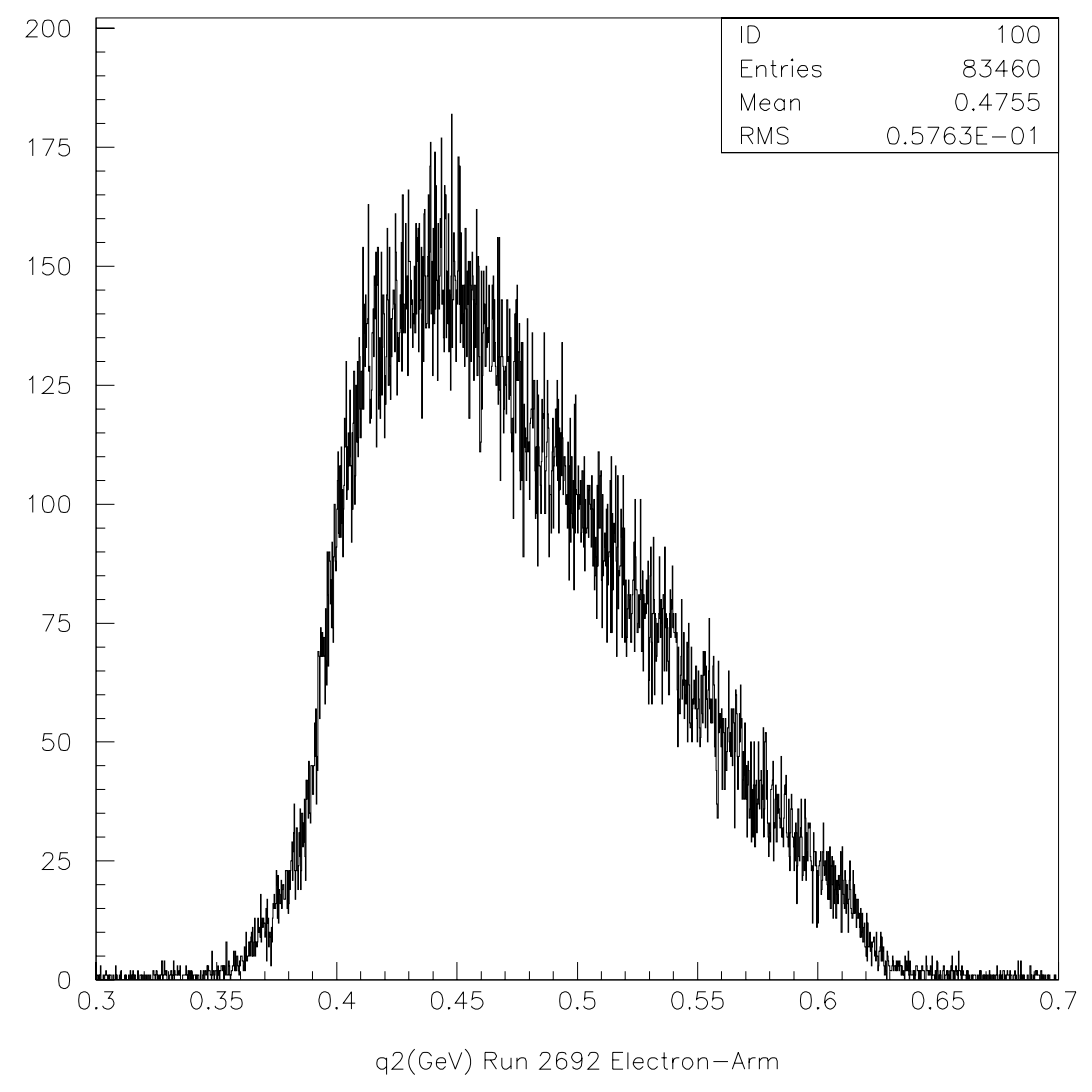

Figure 4.10: Data from an auxiliary run showing the $Q^{2}$ distribution of scattering events intercepted by the HAPPEX detector. The average $Q^{2}$ was determined to be $0.479 \pm 0.003(\mathrm{GeV} / \mathrm{c})^{2}$.

remained on the detector. The integrated detector signals from each spectrometer were compared to determine the probability for inelastic events (which have lower energy and therefore are steered into the walls of the spectrometer) to rescatter into our detector. As a simultaneous parallel measurement, the HAPPEX detector was also used as a trigger for the Hall A DAQ system which measured the scattering rate in our detectors by counting individual events. Since our detector is a total-energy calorimeter, the scattering rate measured by the Hall A DAQ system was weighted by the average energy of the scattered electrons in order to make a valid comparison of the two measurements. 


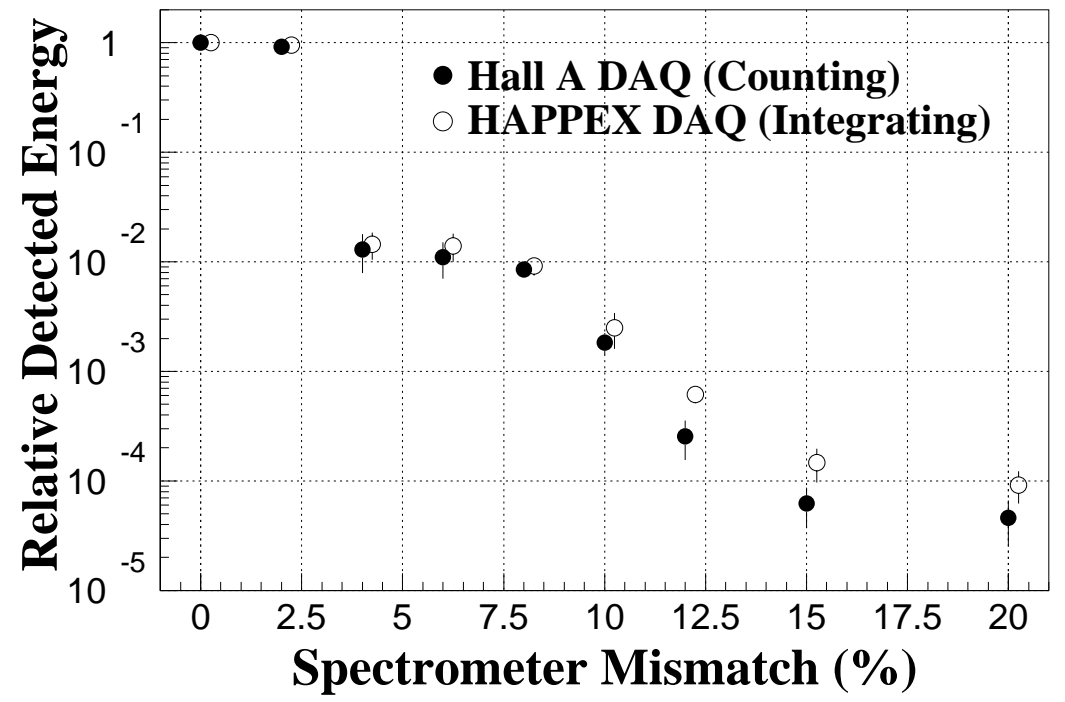

Figure 4.11: Sensitivity of the HAPPEX detector to inelastic events as determined by mistuning one spectrometer. The peak of the $\Delta$-resonance corresponds to a $10 \%$ mismatch.

The results of the test are shown in Figure 4.11. The two methods of measuring the probability of rescattering into the HAPPEX detector are seen to agree well. At a $20 \%$ mismatch of the spectrometer fields, the observed rate falls to $0.01 \%$, demonstrating excellent shielding from room background. The $\Delta$-resonance peaks at a $10 \%$ lower energy than the elastic peak, so we estimate from the plot that $0.2 \%$ of $\Delta$ events rescatter into our detector. The cross section at the $\Delta$-resonance is about twice that for elastic scattering, and the physics asymmetry is estimated to be 2.8 times greater [2]. Assuming these values are uncertain by a factor of three, we conservatively estimate a $2 \%$ systematic error in the final asymmetry due to inelastic background. 


\subsection{Final Experimental Asymmetry}

The average raw asymmetry is $A_{\text {raw }}=-5.64 \pm 0.75 \mathrm{ppm}$. The systematic error in measuring the raw asymmetry is less than $0.1 \mathrm{ppm}$. The experimental asymmetry $A_{e x p}$ is extracted from the raw asymmetry by dividing by the beam polarization $P_{e}$. We used the average of the Mott and Møller measurements, $P_{e}=0.388 \pm 0.027$. The result is

$$
A_{\text {exp }}=-14.5 \pm 2.0(\text { stat }) \pm 1.1 \text { (syst) ppm. }
$$

The systematic error is dominated by the systematic error in the measurement of the beam polarization, which is conservatively estimated to be $7 \%$ of the measured value. A summary of errors is given in Table 4.3.

\begin{tabular}{|l|l|r|}
\hline Asymmetry & Source of error & $\Delta A / A(\%)$ \\
\hline \hline$A_{\text {raw }}$ & Statistics & 13.4 \\
& Others & $<0.3$ \\
\hline$A_{\text {exp }}$ & Beam Polarization & 7 \\
& $Q^{2}$ Determination & 1 \\
& Backgrounds & 2 \\
\hline
\end{tabular}

Table 4.3: Summary of errors for $A_{\text {raw }}$ and $A_{\text {exp }}$. 


\section{Chapter 5}

\section{Conclusion}

We have measured the parity-violating electroweak asymmetry in the elastic scattering of polarized electrons from the proton [31]. The result is

$$
A_{\text {exp }}=-14.5 \pm 2.0(\text { stat }) \pm 1.1(\text { syst }) \mathrm{ppm}
$$

at the kinematic point $\left\langle\theta_{\text {lab }}\right\rangle=12.3^{\circ}$ and $\left\langle Q^{2}\right\rangle=0.48(\mathrm{GeV} / \mathrm{c})^{2}$.

\subsection{Extracting Strange Form Factors}

The theoretical asymmetry is given in the electroweak Standard Model by Equation 1.14, which we repeat here for convenience:

$$
A_{t h}=-\frac{G_{F} Q^{2}}{\pi \alpha \sqrt{2}}\left[\frac{\epsilon G_{E}^{p \gamma} G_{E}^{p Z}+\tau G_{M}^{p \gamma} G_{M}^{p Z}-\frac{1}{2}\left(1-4 \sin ^{2} \theta_{W}\right) \epsilon^{\prime} G_{M}^{p \gamma} G_{A}^{p Z}}{\epsilon\left(G_{E}^{p \gamma}\right)^{2}+\tau\left(G_{M}^{p \gamma}\right)^{2}}\right] .
$$

Under the assumption of strong isospin symmetry, the neutral weak form factors for the proton can be written in terms of the nucleon electromagnetic form factors plus an additional contribution from strange quarks, as discussed in Section 1.2.3:

$$
G_{E, M}^{p Z}=\frac{1}{4}\left[\left(1-4 \sin ^{2} \theta_{W}\right) G_{E, M}^{p \gamma}-G_{E, M}^{n \gamma}-G_{E, M}^{s}\right]
$$

To interpret the experiment in terms of strange quark form factors, we use Equation 5.2 in Equation 5.1 and express the theoretical asymmetry as the sum of 
strangeness-independent and -dependent parts $A_{n s}$ and $A_{s}$ :

$$
\begin{aligned}
A_{t h}= & -\frac{G_{F} Q^{2}}{4 \pi \alpha \sqrt{2}}\left[\left(1-4 \sin ^{2} \theta_{W}\right)-\frac{\epsilon G_{E}^{p \gamma} G_{E}^{n \gamma}+\tau G_{M}^{p \gamma} G_{M}^{n \gamma}+2\left(1-4 \sin ^{2} \theta_{W}\right) \epsilon^{\prime} G_{M}^{p \gamma} G_{A}^{p Z}}{\epsilon\left(G_{E}^{p \gamma}\right)^{2}+\tau\left(G_{M}^{p \gamma}\right)^{2}}\right] \\
& \quad+\frac{G_{F} Q^{2}}{4 \pi \alpha \sqrt{2}}\left[\frac{\epsilon G_{E}^{p \gamma} G_{E}^{s}+\tau G_{M}^{p \gamma} G_{M}^{s}}{\epsilon\left(G_{E}^{p \gamma}\right)^{2}+\tau\left(G_{M}^{p \gamma}\right)^{2}}\right] \\
\equiv & A_{n s}+A_{s}
\end{aligned}
$$

The strangeness-independent part $A_{n s}$ can be evaluated using known values for the form factors. The difference between the experimental asymmetry and $A_{n s}$ is attributed to nonzero strange quark form factors in $A_{s}$.

We evaluate $A_{n s}$ at our $Q^{2}$ using parameterizations of the nucleon electromagnetic form factors. For $G_{E}^{n}$, we use the Galster parameterization given in Equation 1.9. The uncertainty in the Galster approximation is estimated to be $50 \%$ of the Galster function, corresponding to a $9.6 \%$ error in $A_{n s}$. The difference between the true value of $G_{E}^{n}$ and the Galster approximation is denoted by $\delta G_{E}^{n}$. We treat this error separately since it is large and since experiments in progress are expected to improve the uncertainty in $G_{E}^{n}$. For the other electromagnetic form factors $G_{E}^{p}$, $G_{M}^{p}$, and $G_{M}^{n}$, the dipole parameterizations given in Equations 1.7 and 1.8 are taken as reasonable approximations at our $Q^{2}$ [2]. This introduces a relative uncertainty in the theoretical asymmetry of about $4 \%$. Radiative corrections, which contribute a few percent to the asymmetry, were applied $[2,31]$. The axial vector form factor $G_{A}^{Z}$ has large uncertainties in its radiative corrections [2]. Fortunately, the term involving $G_{A}^{Z}$ is suppressed at our kinematics and contributes only a few percent to the asymmetry.

Under these assumptions, the theoretical asymmetry in the absence of strange quarks is given by

$$
A_{n s}=-15.8 \pm 0.7 \pm 1.5\left(\delta G_{E}^{n}\right) \mathrm{ppm} .
$$

Thus $A_{\text {exp }}$ is consistent with $A_{n s}$ with no additional contributions from strange 
quarks. Using the dipole parameterizations for $G_{E, M}^{p \gamma}$, the linear combination of strange quark form factors $G_{E, M}^{s}$ we extract from $A_{s}=A_{\text {exp }}-A_{n s}$ is

$$
G_{E}^{s}+(\tau / \epsilon) \mu_{p} G_{M}^{s}=G_{E}^{s}+0.39 G_{M}^{s} .
$$

The experimentally measured value at $\left\langle Q^{2}\right\rangle=0.48$ is

$$
G_{E}^{s}+0.39 G_{M}^{s}=0.023 \pm 0.034(\text { stat }) \pm 0.022(\text { syst }) \pm 0.026\left(\delta G_{E}^{n}\right),
$$

which is consistent with zero. This result represents the first experimental constraint of $G_{E}^{s}$.

\subsection{Theoretical Implications}

Extensive literature is devoted to estimating the size of the strange form factors. Figure 5.1 shows representative theoretical predictions of $\left(A_{n s}-A_{\text {exp }}\right) / A_{n s}$ using different models for the strange form factors. The data point is our experimental value under the assumption $\delta G_{E}^{n}=0$. The largest of the predictions is excluded by our result. Recent data from a second HAPPEX run at the same kinematics have reduced the error bar on our result with no significant change in the central value.

Most theoretical models of $G_{E}^{s}$ and $G_{M}^{s}$ focus on the leading moments at $Q^{2}=0$. In analogy with the magnetic moment and charge radius of the nucleon, it is customary to define the strange magnetic moment $\mu_{s}$ and strange radius $r_{s}$ at $Q^{2}=0$ :

$$
\mu_{s} \equiv G_{M}^{s}(0) \text { and }\left\langle r_{s}^{2}\right\rangle \equiv-\left.6 \frac{d F_{1}^{s}}{d Q^{2}}\right|_{Q^{2}=0},
$$

where $F_{1}^{s}$ is the Dirac strange form factor. Another commonly used parameter is

$$
\left.\rho_{s} \equiv \frac{d G_{E}^{s}}{d \tau}\right|_{\tau=0}=-\frac{2}{3} m_{N}^{2}\left\langle r_{s}^{2}\right\rangle-\mu_{s}
$$

Since the net strangeness of the nucleon is zero, we have $G_{E}^{s}(0)=0$. 


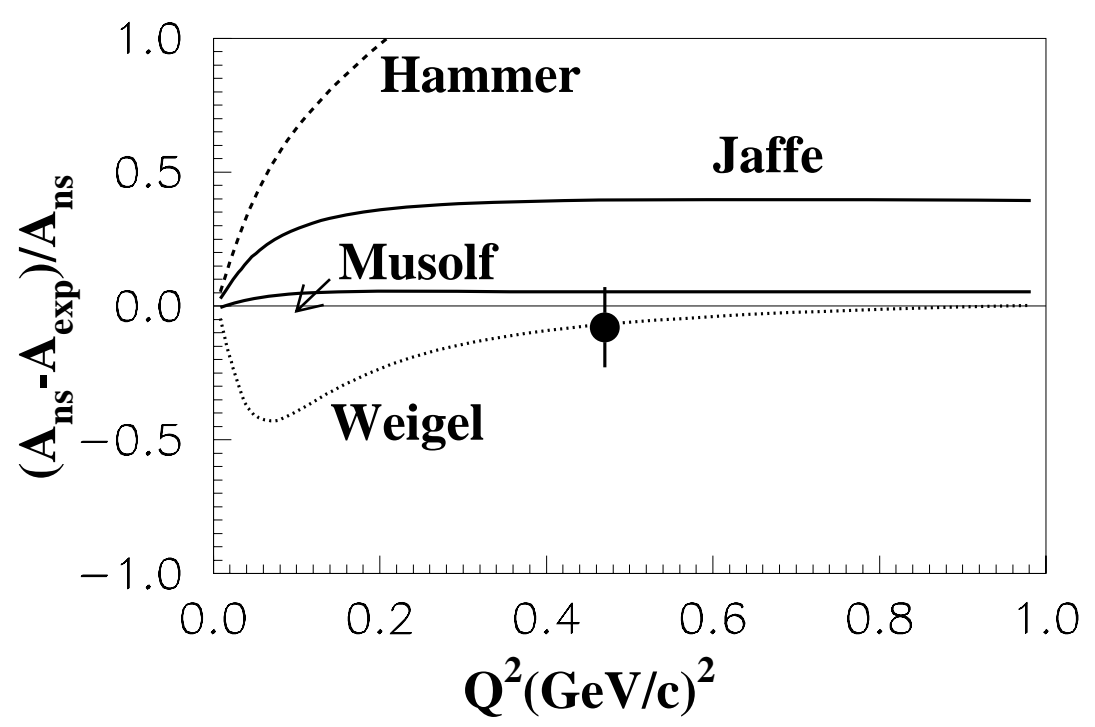

Figure 5.1: Theoretical calculations of $\left(A_{n s}-A_{\text {exp }}\right) / A_{n s}$ for models by Jaffe [32], Hammer et. al. [33], Musolf and Burkardt [34], and Weigel [35]. For models that did not include the $Q^{2}$ dependence $[32,34]$, a dipole form is used as suggested in Reference [2]. The data point is the value from our experiment assuming $\delta G_{E}^{n}=0$.

A sampling of theoretical predictions for $\rho_{s}$ and $\mu_{s}$ using various approaches is shown in Table 5.1. The first approach uses dispersion theory fits to the nucleon isoscalar form factors (pole fits) [32], while the second calculation updates the first to account for new data and constraints from perturbative QCD [33]. Another approach includes kaon loops to quantify the simple picture of nucleon strangeness as arising from a kaon cloud [34]. The Nambu-Jona-Lasino (NJL) model [35], Skyrme model [36], and lattice QCD techniques [37] are other approaches. There also exists a quark model calculation within a framework consistent with the OZI rule [38].

We see that the predictions for $\mu_{s}$ tend to be either small or around -0.3 . Recent data from SAMPLE, however, suggests the surprising result that $\mu_{s}$ may be substantially positive [11]. The predictions for $\rho_{s}$ vary widely and do not agree on the sign. 


\begin{tabular}{lrrl}
\hline Method & $\rho_{s}$ & $\mu_{s}$ & Author \\
\hline Pole fits & -2.1 & -0.31 & Jaffe [32] \\
Pole fits (update) & -2.9 & -0.24 & Hammer et. al. [33] \\
Kaon Loops & 0.5 & -0.35 & Musolf and Burkardt [34] \\
NJL & 3.0 & -0.15 & Weigel et. al. [35] \\
SU(3) Skyrme & 1.6 & -0.13 & Park et. al. [36] \\
Lattice QCD & 1.7 & -0.36 & Dong et. al [37] \\
Quark Model & 0.6 & 0.04 & Geiger and Isgur [38] \\
\hline
\end{tabular}

Table 5.1: Various theoretical predictions for $\rho_{s}$ and $\mu_{s}$.

To confront these predictions with our data, some assumption about the $Q^{2}$ dependence of the form factors must be made. Reference [2] suggests using a Galsterstyle parameterization of the strange form factors which introduces new parameters $\lambda_{E, M}^{s}:$

$$
G_{E}^{s}=\frac{\rho_{s} \tau G_{D}}{1+\lambda_{E}^{s} \tau} ; \quad G_{M}^{s}=\frac{\mu_{s} G_{D}}{1+\lambda_{M}^{s} \tau}
$$

Under the simplifying assumption that $\lambda_{E}^{s}$ and $\lambda_{M}^{s}$ are both zero, our result implies that

$$
\rho_{s}+2.9 \mu_{s}=0.47 \pm 0.83 \pm 0.53
$$

where the first error is experimental, representing statistical and systematic errors added in quadrature, and the second error is due to the uncertainty in $G_{E}^{n}$. This result strongly disfavors the pole fit approach to estimating the strange form factors.

\subsection{Future Directions}

The success of our experiment demonstrates that high luminosity, high accuracy measurements of parity-violating amplitudes are feasible at Jefferson Lab, and has laid the groundwork for future experiments. To date, the HAPPEX and SAMPLE experiments have produced the only measurements of the strange form factors. 
Further experiments are necessary to separate $G_{E}^{s}$ and $G_{M}^{s}$ and determine the $Q^{2}$ dependence of the form factors.

The HAPPEX II experiment at Jefferson Lab proposes to constrain the strange radius of the proton by measuring the electron-proton elastic scattering asymmetry at an angle of $6^{\circ}$ and $Q^{2}=0.1(\mathrm{GeV} / \mathrm{c})^{2}$. Other planned parity-violation experiments include a measurement of elastic scattering from ${ }^{4} \mathrm{He}$ at Jefferson Lab and a measurement of $F_{1}^{s}$ through electron-proton scattering at Mainz. The $G^{0}$ experiment in Hall $\mathrm{C}$ of Jefferson Lab plans to make a complete set of asymmetry measurements for $0.16<Q^{2}<0.95(\mathrm{GeV} / \mathrm{c})^{2}$ at both forward and backward angles. 
Appendix A

Electronics Handbook 


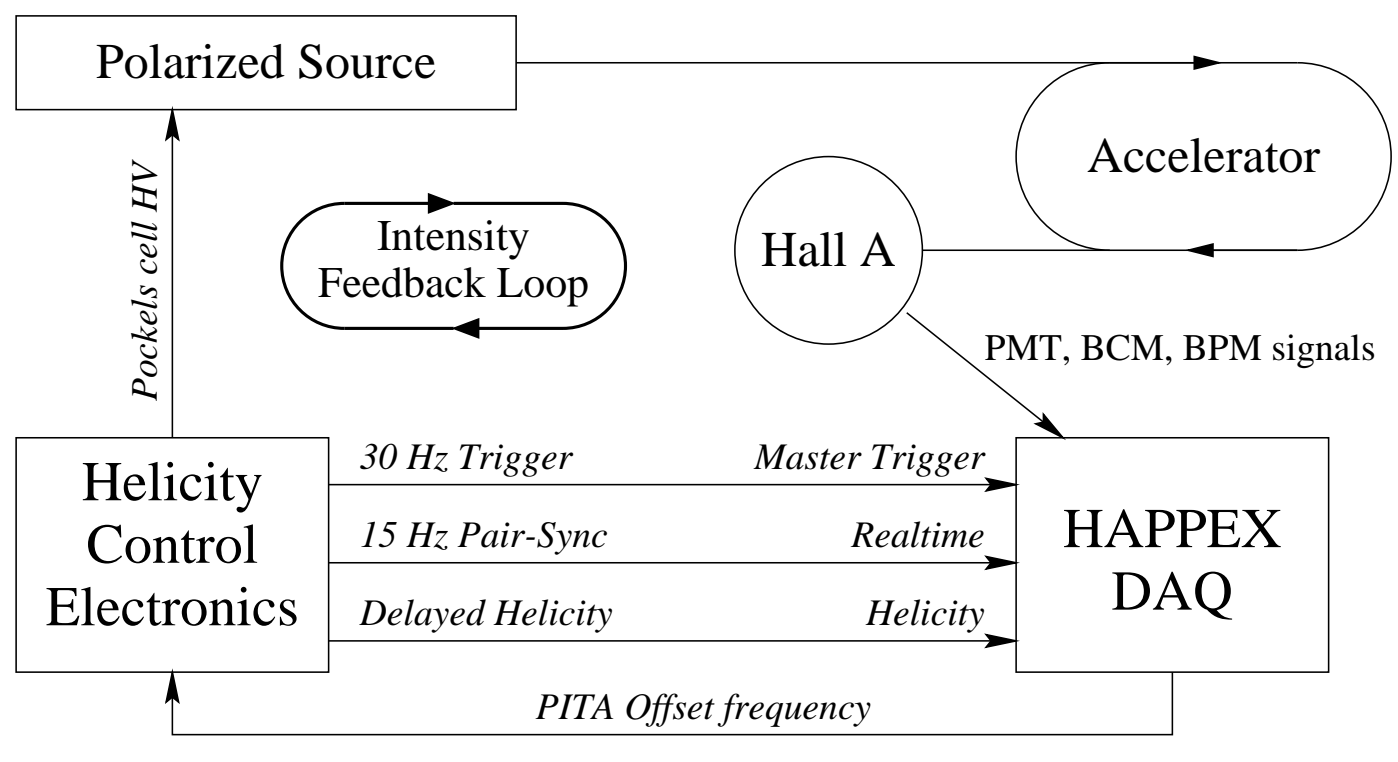

Optical Fibers

Figure A.1: Overview of custom electronics systems and their interconnections.

\section{A.1 Overview}

This document provides a detailed description of the custom electronics built for the HAPPEX experiment at Jefferson Lab. There are two major systems: the helicity control electronics and the HAPPEX data acquisition (DAQ) electronics, shown in Figure A.1. The helicity control electronics, located at the polarized source, control the polarization of the source laser and thereby control the helicity of the electron beam. The HAPPEX DAQ electronics, located in the Hall A counting house, integrate analog signals from the particle detectors and beam monitors and digitize the integrated results. Signals are exchanged over optical fibers in order to maintain maximum electrical isolation between the two systems. 


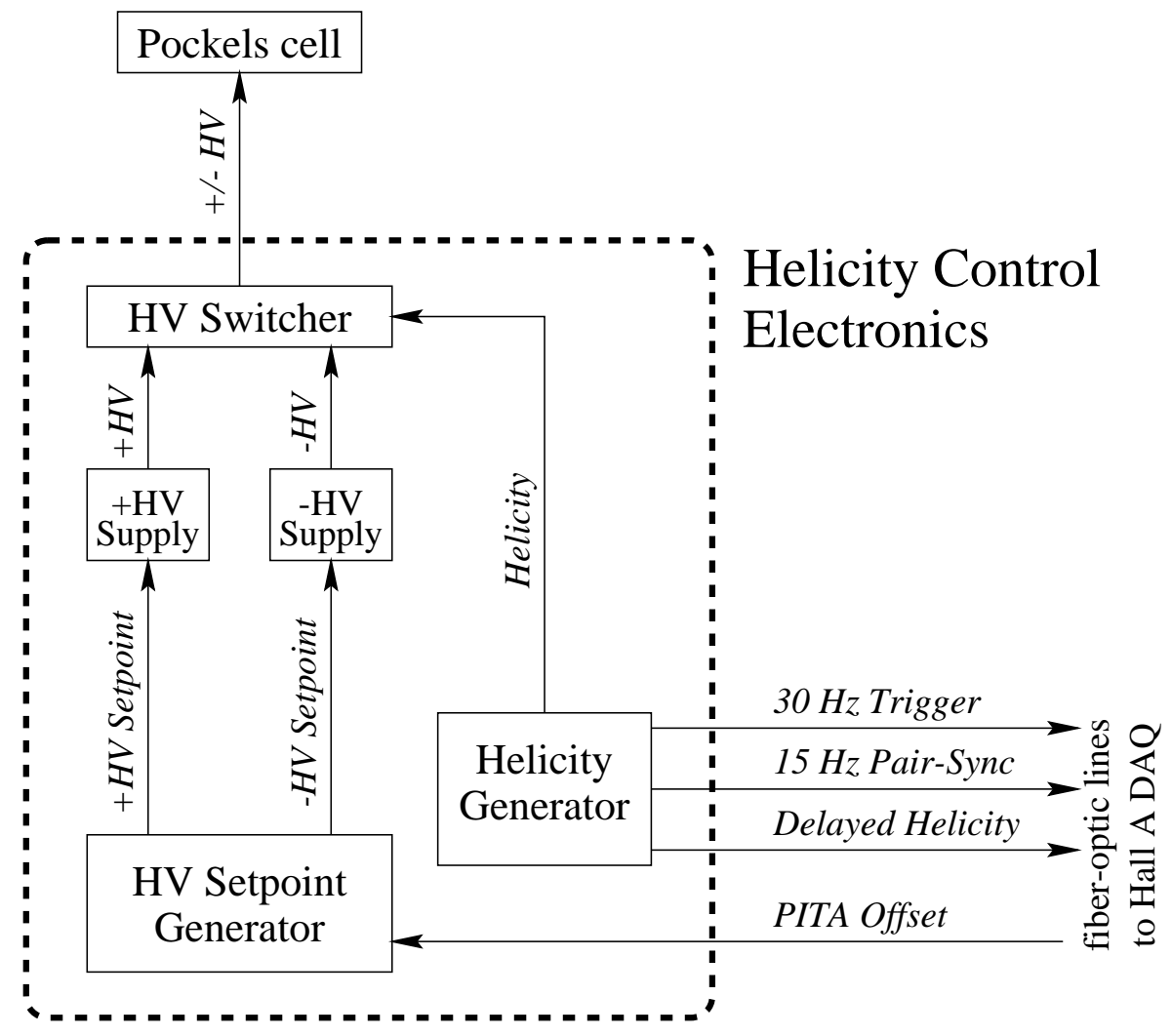

Figure A.2: Block diagram of helicity control electronics.

\section{A.2 Helicity Control Electronics}

A diagram of the helicity control electronics is shown in Figure A.2. The Pockels cell High Voltage Supplies and Switcher are located down in the tunnel near the polarized source laser table. The Helicity Generator and High Voltage Setpoint Generator are located directly above the polarized source in the Injector Service Building.

The helicity control electronics control the helicity of the electron beam. The polarized electron beam originates from a GaAs photocathode excited by circularly polarized laser light. The helicity of the electron beam can be reversed by reversing the handedness of the circular polarization. Thus the task of setting the helicity of 
the electron beam is reduced to the task of controlling the circular polarization of the laser.

Circularly polarized laser light is generated from linearly polarized laser light using a voltage-controlled phase retarder known as a Pockels cell. The Pockels cell is aligned so that $\pm \lambda / 4$ retardation yields either right- or left-circular polarization. The HV Switcher provides the Pockels cell with either positive or negative high voltage depending on the state of a TTL digital control signal. When the control signal is high (low), the switch connects voltage from a positive (negative) HV supply to the Pockels cell. The programmable $\pm \mathrm{HV}$ supplies are set to supply voltages corresponding to $\pm \lambda / 4$ retardation, which is approximately $\pm 2.5 \mathrm{kV}$. The net effect of the entire system is that the helicity of the electron beam depends on the state of the digital control signal, or Helicity signal. The Helicity signal is provided by the Helicity Generator, which thereby controls the helicity sequence and timing structure of the polarized electron beam.

The system is complicated somewhat by the implementation of intensity feedback, which requires the ability to introduce a small antisymmetric offset to the Pockels cell quarter-wave voltages. This requirement is incorporated into the HV Setpoint Generator, which supplies the programming voltages to the HV supplies. The size of the voltage offset is determined by the PITA Offset signal provided by the DAQ system in Hall A.

The following sections will describe the various components of the helicity control electronics in greater detail.

\section{A.2.1 High Voltage Supplies and Switcher}

The High Voltage Supplies and Switcher provide high voltage to the Pockels cell in order to convert linearly polarized laser light to circularly polarized laser light. 
There are two HV supplies, one positive and one negative. The $\pm H V$ supplies used for HAPPEX are programmable DC-to-DC converters manufactured by Emco. Each supply's $0-4 \mathrm{kV}$ output range is spanned by a $0-10 \mathrm{~V}$ programming voltage supplied by the HV Setpoint Generator, described in Section A.2.3 below. The \pm HV Supply outputs are fed to the HV Switcher, which connects either the positive or negative high voltage to the Pockels cell depending on the state of the Helicity signal from the Helicity Generator. Switching is performed by Behlke HTS-121 high voltage switches.

\section{A.2.2 Helicity Generator}

The Helicity Generator dictates the time structure and helicity sequence of the polarized electron beam. The helicity is set every $33.3 \mathrm{~ms}$ locked to the $60 \mathrm{~Hz} \mathrm{AC}$ power line. Each 33.3 period of constant helicity is called a window. A $15 \mathrm{~Hz}$ pair structure is also imposed, in which the windows are generated in pairs with opposite helicity. The helicity of the first window of a pair is chosen by a pseudorandom bit generator, and the second window is given the opposite helicity. A circuit diagram of the Helicity Generator is shown in Figure A.3.

\section{Instructions for Using the Helicity Generator}

The Helicity Generator has no inputs except the AC power line and produces four outputs, described in Section A.2.2 below. The Helicity output signal is sent via optical fiber to the HV Switcher in order to set the helicity of the electron beam. The other three output signals are sent via optical fibers to the DAQ system in Hall A. TTL versions of all four output signals are provided on BNC connectors for diagnostic purposes only. 


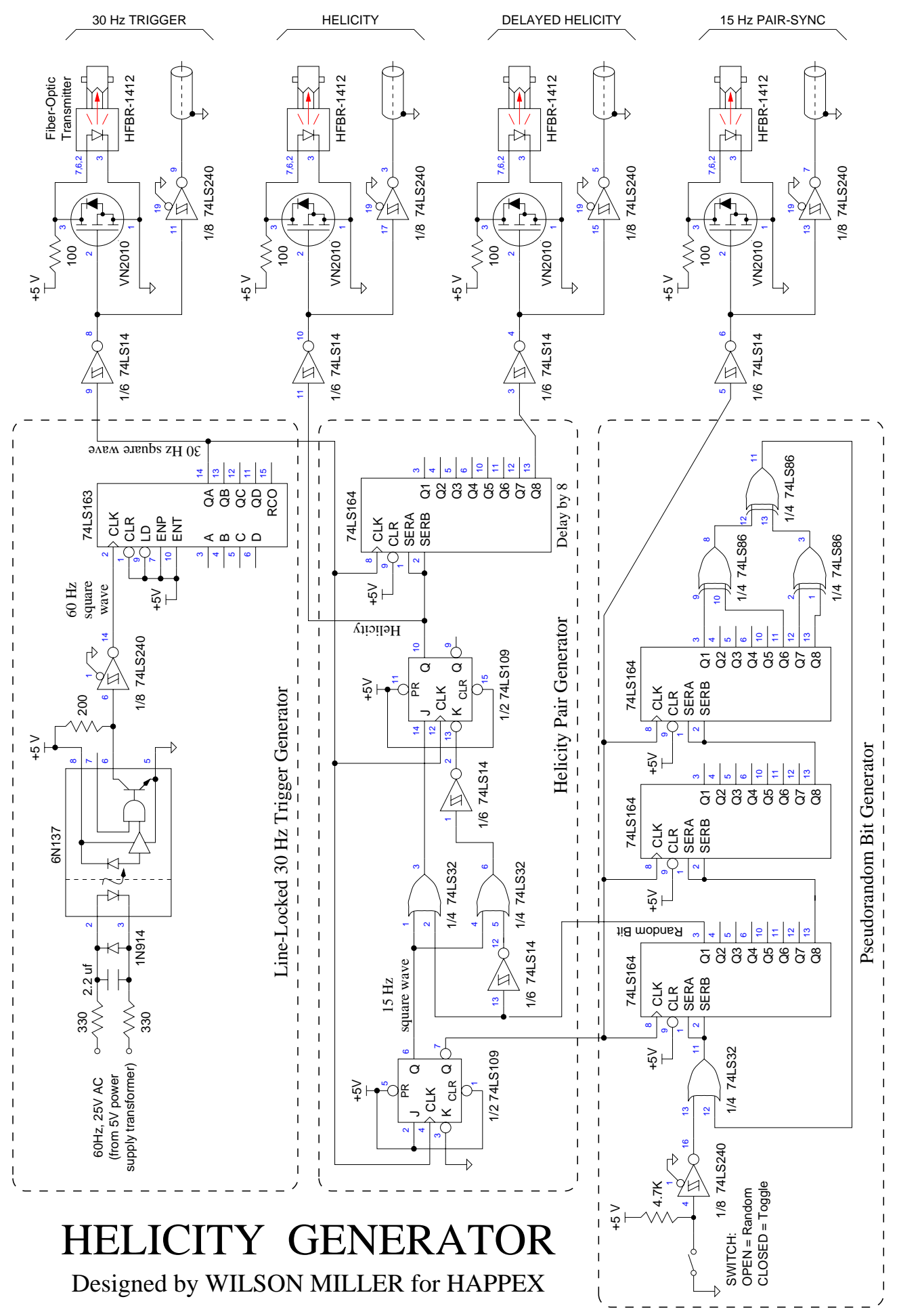

Figure A.3: Circuit diagram of Helicity Generator. 


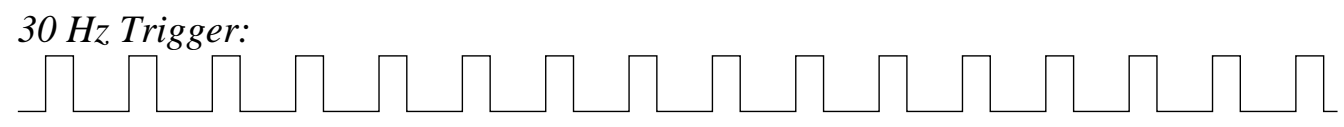

$15 \mathrm{~Hz}$ Pair-Sync:

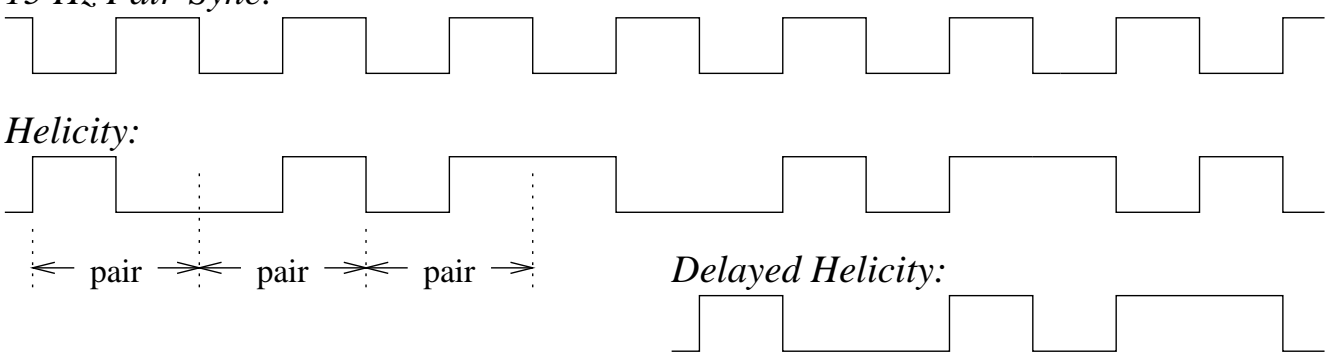

Figure A.4: Sample timing diagram for Helicity Generator outputs. Note the pseudorandom pair structure in the Helicity signal. The Delayed Helicity signal is just the Helicity signal delayed by eight ticks of the $30 \mathrm{~Hz}$ Trigger.

The Helicity Generator may be operated in one of two modes controlled by a switch on the front panel. In random mode, the pair-wise pseudorandom helicity sequence described above is generated. In toggle mode, the pseudorandom bit generator is disabled and the helicity simply toggles every $33.3 \mathrm{~ms}$. The switch must be moved to toggle immediately after cycling the power in order to seed the pseudorandom bit generator, as described in Section A.2.2. If random mode is desired, the switch may be moved back to random after a few seconds in toggle mode.

\section{Description of the Output Signals}

A sample timing diagram for the Helicity Generator's four output signals is shown in Figure A.4.

$30 \mathrm{~Hz}$ Trigger The $30 \mathrm{~Hz}$ Trigger signal is phase-locked to the $60 \mathrm{~Hz}$ power line. Each rising edge of the $30 \mathrm{~Hz}$ Trigger signal marks the beginning of a helicity window. This signal is sent via optical fiber to the Hall A counting house, where it serves as the Master Trigger for the integration cycle. 
$15 \mathrm{~Hz}$ Pair-Sync The $15 \mathrm{~Hz}$ Pair-Sync signal toggles at each rising edge of the $30 \mathrm{~Hz}$ Trigger. It divides the $30 \mathrm{~Hz}$ windows into pairs. Fiber off (on) indicates the first (second) window of a pair. This signal is sent via optical fiber to the Hall A counting house where it is known as Realtime and lets the DAQ system know whether the present window is the first or second of a pair.

Helicity The Helicity signal represents the present helicity of the electron beam. The first window of a pair is chosen by a pseudorandom bit generator, described in Section A.2.2 below. The second window of a pair is given the opposite helicity of the first. The Helicity signal is sent only to the HV Switcher and nowhere else, because the present helicity must not be known outside the source in order to avoid false asymmetries due to electronic cross-talk.

Delayed Helicity The Delayed Helicity signal is identical to the Helicity signal, except that it is delayed by exactly eight ticks of the $30 \mathrm{~Hz}$ Trigger. Thus it has the same phase as the present helicity with respect to the $15 \mathrm{~Hz}$ Pair-Sync signal but comes four window pairs later. The Delayed Helicity signal is sent via optical fiber to the Hall A counting house where it is recorded by the DAQ system and used to reconstruct the correct helicity for each window.

\section{Pseudorandom Bit Generator}

The pseudorandom bit generator is a 24-bit shift register with feedback taps on bits 17, 22, 23, and 24, as shown in Figure A.5. The shift register is clocked by the 15 Hz Pair-Sync signal, so it generates a new random bit for each window pair. At the rising edge of the clock, all bits are shifted one position to the right, and a pseudorandom bit, formed by the logical exclusive-or of bits $17,22,23$, and 24 , is 
Clock

$(15 \mathrm{~Hz}$

Pair-Sync)

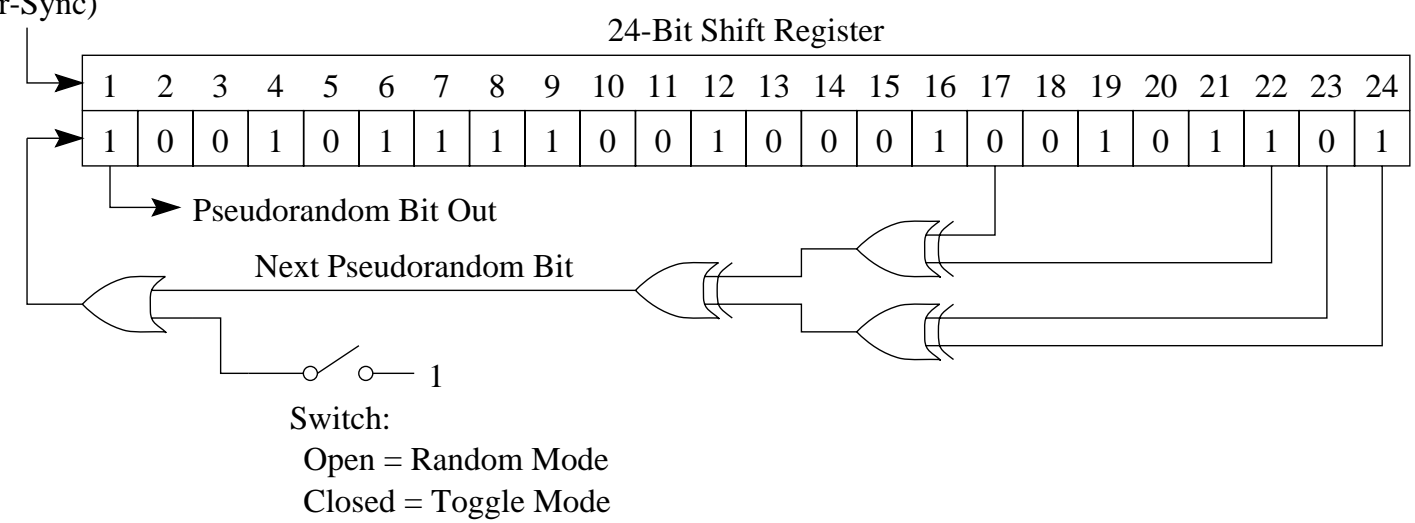

Figure A.5: Pseudorandom bit generator.

loaded into bit 1. This particular combination of feedback bits is chosen to generate a pseudorandom sequence of maximal length. That is, if the shift register is thought of as a 24 -bit state machine, all $2^{24}$ possible states are visited before repeating. Since the shift register is clocked at $15 \mathrm{~Hz}$, the pseudorandom sequence repeats after about 13 days.

Notice that if the shift register starts out in the state with all 0s, it will remain in that state forever, since the exclusive-or of zeros is always zero. (Thus there are actually only $2^{24}-1$ states in the maximal-length sequence.) If there is at least one 1 bit somewhere in the shift register, each of the $2^{24}-1$ states will eventually be reached, and the desired pseudorandom sequence will be generated. The problem is that the circuit usually powers up with all 0s. For this reason, a manual switch is provided to seed the shift register with 1s. When the switch is closed, the exclusiveor of the feedback taps is overridden and a 1 is automatically presented to the input of the shift register. The switch must be closed briefly upon powering up the module and must remain closed for at least one clock tick (1/15 s) in order to load at least one 1 into the shift register. 


\section{Running in Toggle Mode}

Also note that if the switch is left closed, the pseudorandom bit generator will produce a stream of $1 \mathrm{~s}$, and the helicity sequence will not consist of random pairs but instead will toggle between 1 and 0 until the switch is opened. Sometimes it is desirable for the helicity to toggle between 1 and 0 , so the switch can intentionally be kept closed to run in "toggle mode."

One word of caution: When operating in toggle mode, the $15 \mathrm{~Hz}$ Pair-Sync signal should never be taken to represent the helicity of the beam, even though it toggles like the Helicity signal in this mode. The reason for this caution is due to a subtle peculiarity of the circuit. If the circuit is powered up with the switch open, and the switch is not subsequently closed briefly to seed the pseudorandom bit generator with 1s, then the pseudorandom bit generator will produce a stream of zeros, and the helicity will toggle between 0 and 1 (instead of 1 and 0 ). The helicity sequence will appear to be in toggle mode, but the $15 \mathrm{~Hz}$ Pair-Sync signal will be out of phase with the Helicity signal, whereas in true toggle mode the two signals are identical. This situation has occurred during Moller polarization measurements and created much confusion when the polarization appeared to have the incorrect sign.

\section{Predicting the Pseudorandom Bit Sequence}

Since the shift-register algorithm used to produce the pseudorandom bit sequence is known, the next bit in the sequence can be predicted by online software and used to verify that the helicity sequence is being tracked correctly. A C subroutine which implements the shift-register algorithm is included below. ${ }^{1}$ It begins correctly

\footnotetext{
${ }^{1}$ Courtesy Richard Holmes, Syracuse University.
} 
predicting the next bit only after the first 24 bits in the sequence have been loaded into the shift register.

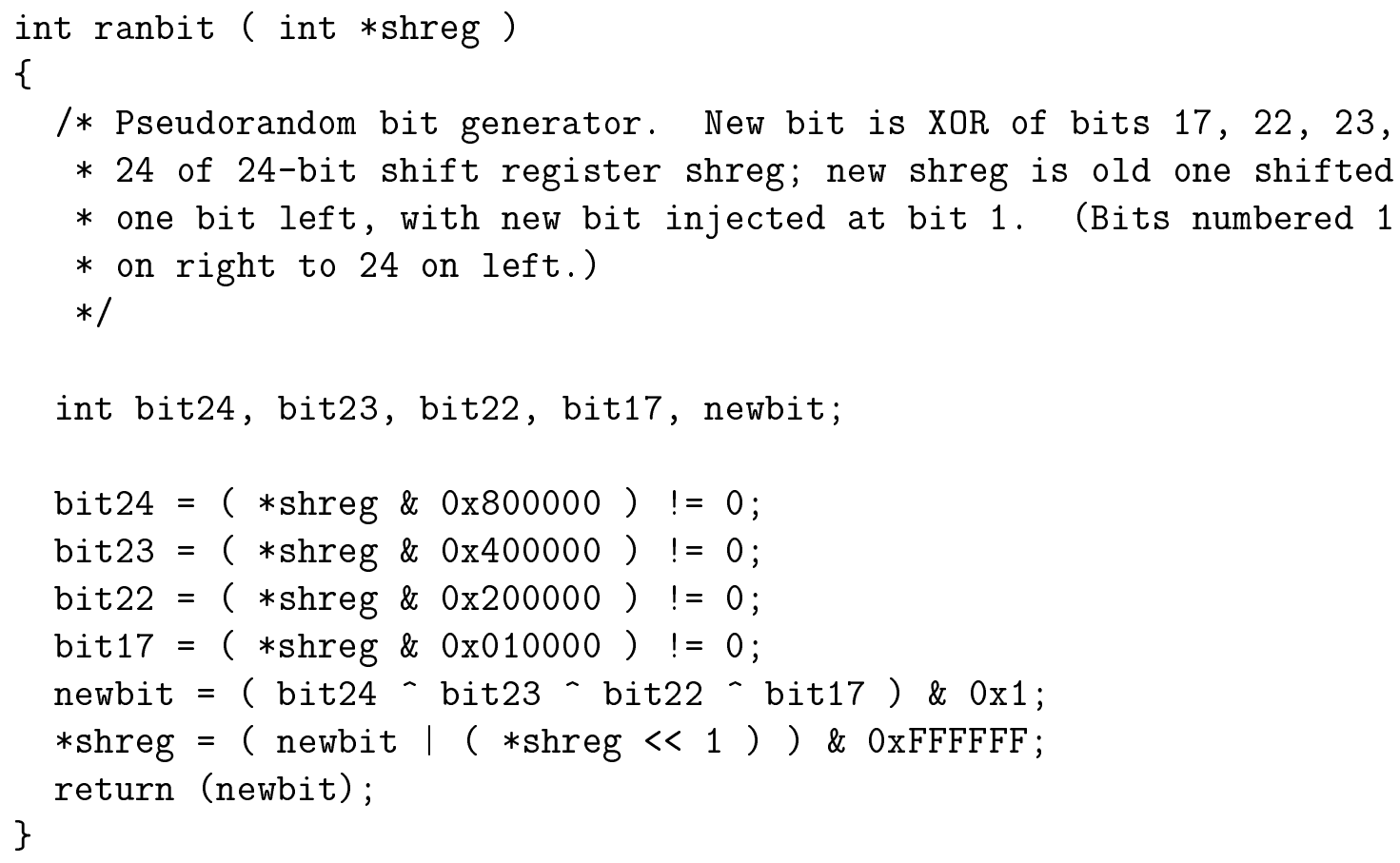

If this algorithm does not correctly reproduce the pseudorandom bit stream seen by the DAQ system, it is possible that the DAQ system is receiving an inverted version of the helicity. (For accounting purposes, it does not matter whether a + helicity is denoted by a 1 or a 0 . It does matter when trying to reproduce the shift register sequence!) Try inverting the helicity bit in the data stream before applying the algorithm and see if that solves the problem. Also remember that this algorithm should be applied to every other bit in the helicity sequence, since only the first window in each pair has random helicity.

\section{A.2.3 HV Setpoint Generator}

The HV Setpoint Generator provides the programming voltages for the two Pockels cell high voltage supplies. The programming voltages corresponding to $\pm \lambda / 4$ re- 
tardation are set using dials on the front panel of the HV Setpoint Generator. An antisymmetric offset can be added to the $\pm \lambda / 4$ setpoints as part of the intensity feedback loop. The magnitude of this offset is determined by the frequency of the PITA Offset signal, supplied by the Hall A DAQ system.

\section{Instructions for Using the HV Setpoint Generator}

The HV Setpoint Generator has one fiber-optic input, two input dials, and two output voltage signals. The two output signals should be connected to the programming inputs of the $\pm \mathrm{HV}$ supplies. The PITA Offset signal from the Hall A DAQ system should be connected to the fiber-optic input. The Pockels cell $\pm \lambda / 4$ voltages are set using the two input dials. The PITA Offset signal should be disconnected (or set to $50 \mathrm{kHz}$ ) while setting the quarter-wave voltages.

There are two diagnostic BNC outputs provided on the front panel. One gives a TTL copy of the PITA Offset frequency input, so that it can be observed on an oscilloscope. The other gives an analog copy of the output of the frequency-tovoltage converter, which maps input frequencies of $0-100 \mathrm{kHz}$ to output voltages of $0-10 \mathrm{~V}$. It should read $5.0 \mathrm{~V}$ for an input of $50 \mathrm{kHz}$.

\section{Functional Details}

Each $\pm \mathrm{HV}$ supply takes a programming voltage $V_{ \pm}$in the range 0 to $10 \mathrm{~V}$ and maps this to an output high voltage $H V_{ \pm}$in the range 0 to $\pm 4 \mathrm{kV}$. Thus the voltages supplied to the Pockels cell are given by

$$
\begin{aligned}
& H V_{+}=+\left(V_{+} \cdot 400 \text { Volts }\right), \\
& H V_{-}=-\left(V_{-} \cdot 400 \text { Volts }\right) .
\end{aligned}
$$


The programming voltages $V_{ \pm \lambda / 4}$ corresponding to $\pm \lambda / 4$ retardation are set using dials on the front panel. The minimum setting on the dial gives a programming voltage of $0 \mathrm{~V}$, while the maximum setting gives $10 \mathrm{~V}$. Hence the dials span the full output range of the high voltage supplies. For HAPPEX, the dials were set to about $62.5 \%$ of maximum, since the Pockels cell quarter-wave voltage was about $2500 \mathrm{~V}$.

An offset voltage $V_{\Delta}$ can be added antisymmetrically to the $\pm \lambda / 4$ setpoints as part of the intensity feedback loop. The magnitude of this offset is determined by the frequency of the PITA Offset signal, supplied by the Hall A DAQ system. A frequency of $50 \mathrm{kHz}$ produces zero offset, and frequencies in the range $25-75 \mathrm{kHz}$ produce offsets of -262 to $262 \mathrm{mV} .^{2}$ (This corresponds to offsets on the high voltage supply outputs of -105 to $105 \mathrm{~V}$.) The voltage offset is added to the quarter-wave programming voltage for the $+\mathrm{HV}$ supply and subtracted from the quarter-wave programming voltage for the - HV supply. That is, we have

$$
\begin{aligned}
& V_{+}=V_{+\lambda / 4}+V_{\Delta}, \\
& V_{-}=V_{-\lambda / 4}-V_{\Delta} .
\end{aligned}
$$

The HV Setpoint Generator contains a "smart" circuit which recognizes whether there is a frequency present on the PITA Offset input. When a frequency above $2 \mathrm{kHz}$ is detected, an LED on the front panel lights up and the PITA offset voltage is applied to the quarter-wave programming voltages. When there is no frequency present, no offset is applied. Without this feature, a frequency of $0 \mathrm{kHz}$ would cause the maximum offset to be applied. This smart feature serves three purposes:

1. In case the PITA Offset frequency generator in the Hall A DAQ system fails or the cable is disconnected, the voltage offset reverts to zero rather than assuming a highly-skewed value.

\footnotetext{
${ }^{2}$ The PITA offset circuit is nonlinear for frequencies above $80 \mathrm{kHz}$, so frequencies outside the range $25-75 \mathrm{kHz}$ should not be used.
} 


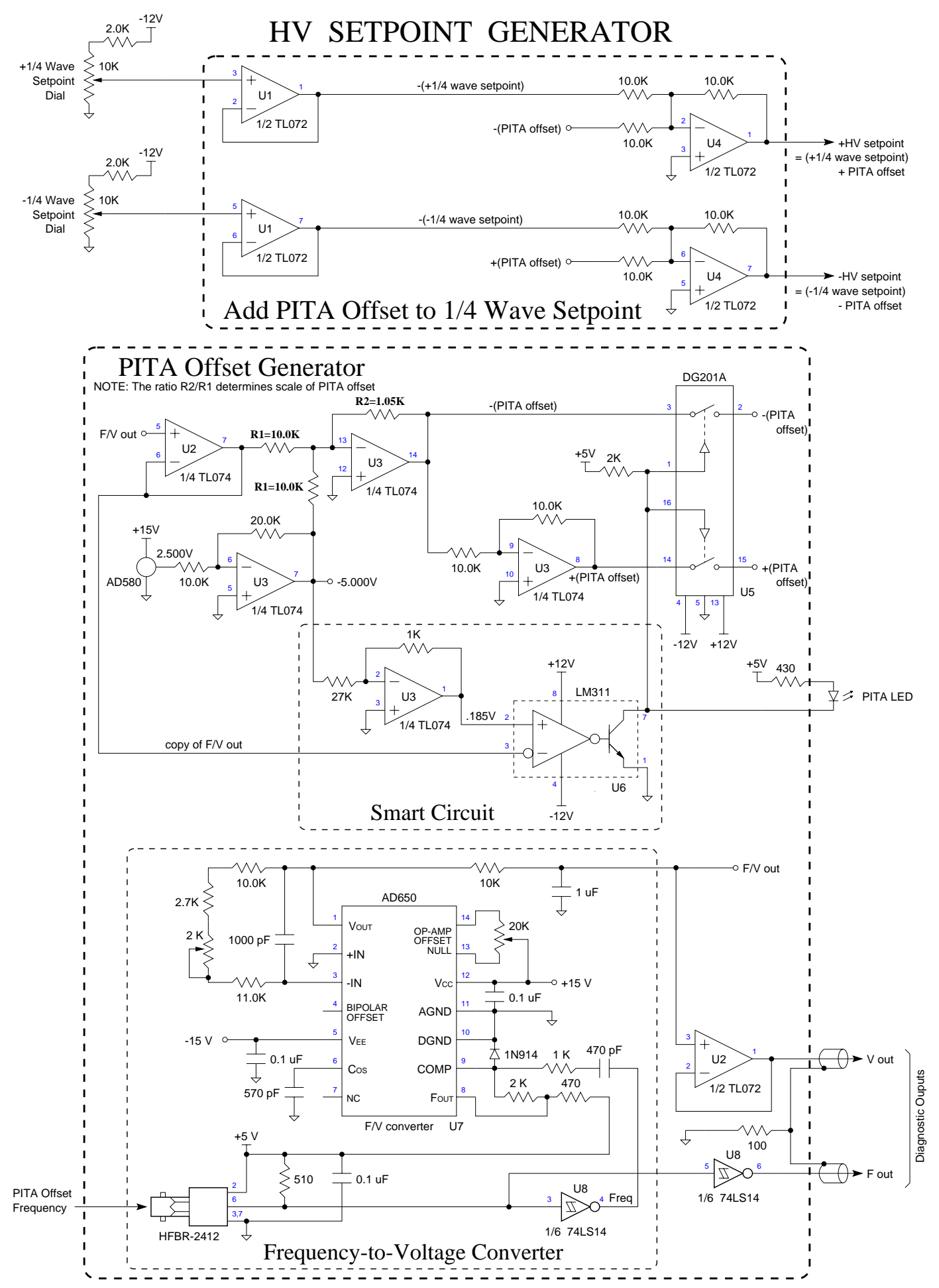

Figure A.6: Circuit diagram of HV Setpoint Generator. 
2. The quarter-wave voltages can be set without first manually setting the PITA Offset frequency to $50 \mathrm{kHz}$. Instead, the fiber-optic cable can simply be removed while the quarter-wave voltages are set.

3. The HV Setpoint Generator can be used to set the Pockels cell voltages in the absence of intensity feedback.

A circuit diagram of the HV Setpoint/PITA Offset Box is shown in Figure A.6. The scale of the PITA offset is determined by the ratio of resistors R2 and R1. The value of R2 may be changed to adjust the range of the PITA offset.

\section{A.3 Data Acquisition Electronics}

The primary function of the Data Acquisition (DAQ) electronics is to integrate and digitize analog signals from the particle detectors and beam monitors. All signals are integrated for $32 \mathrm{~ms}$ starting about $1 \mathrm{~ms}$ after the beginning of a helicity window. The beginning of a helicity window is indicated by the rising edge of the $30 \mathrm{~Hz}$ Trigger signal from the helicity control electronics. The DAQ electronics also process the helicity information from the helicity control electronics and generate the PITA Offset frequency signal as part of the intensity feedback loop.

A diagram of the DAQ system is shown in Figure A.7. All modules are housed in a VME crate, which allows them to communicate with one another across the VMEbus backplane. The ADC boards perform the integration and digitization. The ADC Timing Board provides timing signals to the ADC boards and triggers the VME Trigger Interface module when there is valid ADC data to be read. It also receives the Delayed Helicity and $15 \mathrm{~Hz}$ Pair-Sync signals from the helicity control electronics and generates the PITA Offset frequency signal. The VME computer 


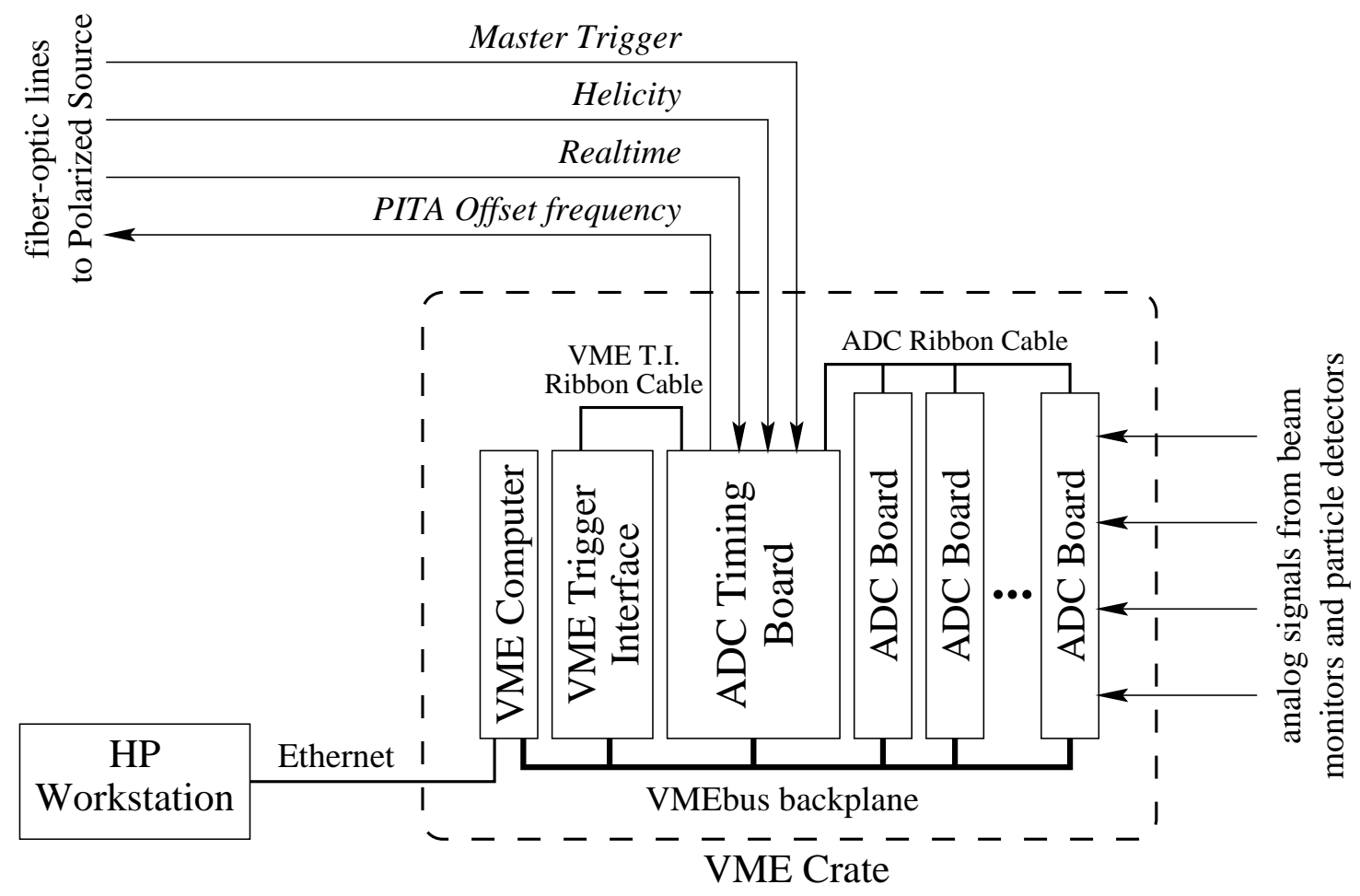

Figure A.7: Data acquisition electronics.

collects data from the VME Trigger Interface and ADC boards and sends the data via ethernet to a Hewlett Packard workstation for storage and analysis.

The following sections will describe the ADC boards and the ADC Timing Board, which were custom-built for the HAPPEX experiment.

\section{A.3.1 ADC Boards}

The heart of the HAPPEX DAQ system are custom integrating analog-to-digital converter (ADC) boards built by John Oliver at the Harvard University High Energy Physics Laboratory. They are designed to integrate analog signals for repetitive fixed intervals and to digitize them to high resolution and low differential nonlinearity. These design goals are achieved using a single-slope sampling technique along with the use of pseudorandom noise injected onto the signal before digitization [22]. Each 


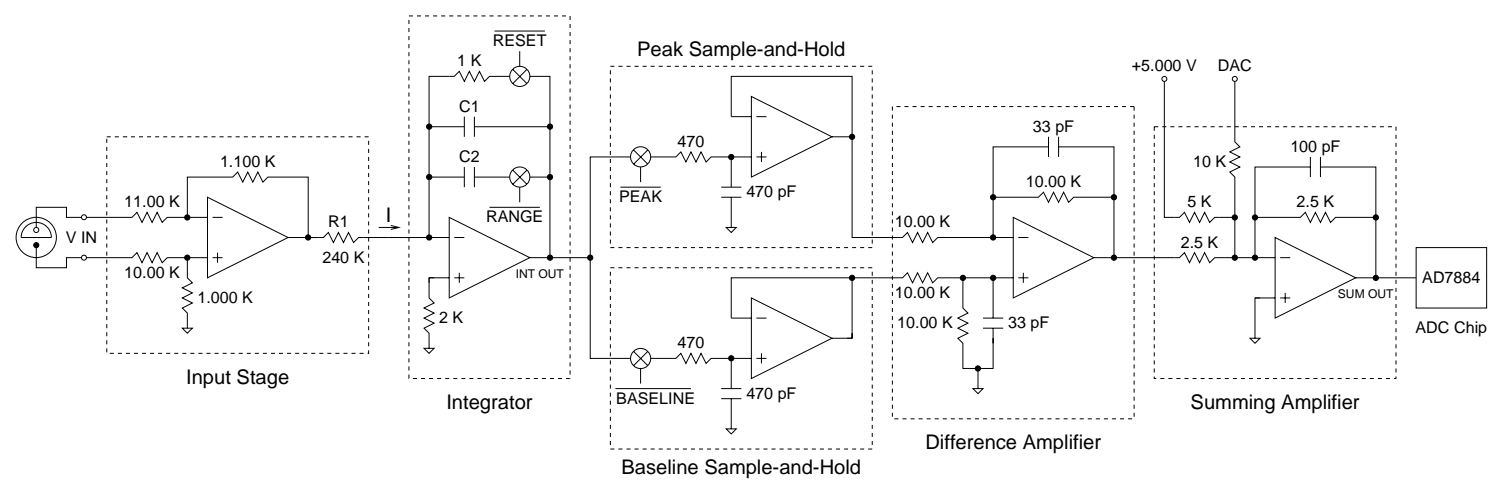

Figure A.8: Circuit diagram of an ADC channel. A voltage-integrating channel is shown here.

board has four ADC channels which can be made voltage- or current-integrating with minor board modifications.

The following sections describe in detail the operating principles, performance characteristics, and instructions for using the ADC boards.

\section{Principles of Operation}

Each ADC channel consists of an input stage, an integrator with reset switch, two sample-and-hold circuits, a difference amplifier, a summing amplifier, and a 16-bit ADC chip, as shown in Figure A.8. For voltage-integrating channels, the input stage is a balanced-input differential amplifier followed by a resistor. It converts the input voltage signal to an appropriately-scaled current which charges the integrating capacitors $\mathrm{C} 1$ and $\mathrm{C} 2$. For current-integrating channels, the differential amplifier is bypassed and the input current is integrated directly. Two different gain ranges are available by switching capacitor $\mathrm{C} 2$ in or out of the integrator circuit.

For a constant input signal, the voltage across the integrating capacitor ramps up linearly. This voltage ramp is sampled and held at two points. The baseline sample is collected at the beginning of the integration period, and the peak sample 
is collected at the end of the integration period. The difference between these two samples is the integrated result. The difference amplifier passes the integrated result to the summing amplifier, where a -2.5 volt offset and programmable DAC voltage are added in. Finally, the output of the summing amplifier is passed to the ADC chip for digitization.

The ADC chip converts an analog input in the range -3 to +3 volts to a 16-bit digital value. The -2.5 volt offset puts the zero-point of the integrated result 0.5 volts above the bottom of this range. Placing the zero point at -2.5 volts instead of -3.0 volts allows signals near zero to be integrated without danger of saturation at the lower end of the ADC chip's input range. The integrated result may not exceed 5.5 volts or else saturation will occur at the upper end of the range. The integrator gain determines the size of the integrated result for a given input signal. Changing the integrator gain is discussed in Section A.3.1.

The output of the 16-bit DAC is scaled to span the range -77 to $+77 \mathrm{mV}$ at the output of the summing amplifier. Thus it spans 1700 ADC bins or about 11 bits. The DAC is used to add a pseudorandom voltage offset to the integrated result before digitization. This pseudorandom voltage is referred to as "DAC noise." The $\mathrm{ADC}$ response to the DAC is calibrated in advance and then the DAC contribution to the digitized result is subtracted during analysis. The use of DAC noise drastically reduces the differential nonlinearity of the ADC by averaging over the nonlinearities of 11 ADC bits.

Timing of the Integration Cycle The beginning, end, and duration of an integration cycle are determined by timing signals common to all four ADC channels: Reset, Baseline, Peak, and Convst. The relative timing of these signals is shown in Figure A.9. These signals are provided to each ADC board by the ADC Tim- 


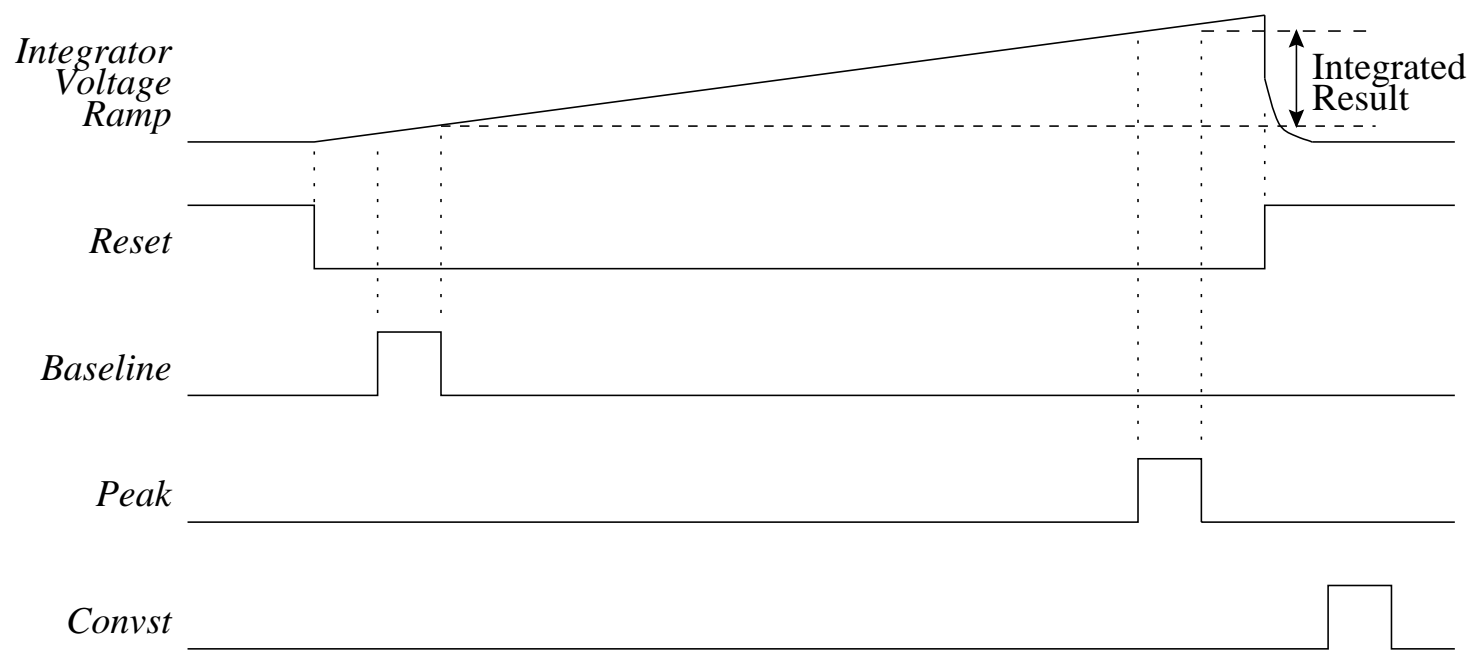

Figure A.9: Timing diagram for ADC integration cycle.

ing Board described in Section A.3.2. Here we describe the function and timing requirements of each of the timing signals.

While Reset is asserted, the reset switch is closed and the integrating capacitor discharges through a $1 \mathrm{k} \Omega$ resistor (see Figure A.8). Upon release of Reset, the input current begins charging the integrating capacitor. The integrator is allowed to settle for about $15 \mu$ s before briefly asserting Baseline to collect the baseline sample. This marks the beginning of the integration interval. At the end of the integration interval, Peak is briefly asserted to collect the peak sample. The width of Baseline and Peak determine the sampling time for their respective sample-andhold circuits, while the delay between Baseline and Peak determines the actual integration time. The optimal width for Baseline and Peak is on the order of a microsecond, while the delay between them can be set to any desired integration time. As soon as the Peak sample has been collected, Reset is asserted once again to clear the integrator, and remains asserted until the beginning of the next integration cycle. 
Next the Convst signal is strobed to start digitization. A suitable delay between Peak and Convst is required in order to allow the difference and summing amplifiers to settle to their new values before digitization. The ADC board is designed to integrate signals which do not change much between consecutive cycles, and therefore the operational amplifiers and feedback capacitors used in the difference and summing circuits have been chosen for low noise rather than high speed. It has been determined empirically that a delay of $22 \mu$ s between Peak and Convst will suffice for integrated signals which vary less than $50 \%$ of full scale.

Upon completion of the integration cycle, the digitized values can be read out over the VMEbus backplane. The new DAC value for the next integration cycle should also be set at this time in order to give the DAC and summing amplifier plenty of time to settle before the next conversion.

\section{ADC Performance}

Resolution The resolution of the ADC channel is limited by pedestal noise arising from the ADC chip, op-amps, and timing jitter. Pedestal noise is measured by examining ADC response for zero input signal. A histogram of pedestal noise is shown in Figure A.10. Pedestal noise is typically 1.5 ADC bins (rms) although it varies by a few tenths of a bin from channel to channel. The use of DAC noise does not increase pedestal noise. Since pedestal noise is independent of the size of the integrated signal, the signal-to-noise ratio can be maximized by using the full dynamic range of the ADC channel. For example, the pedestal noise is only $0.003 \%$ for a signal of 50,000 ADC bins.

Integral Nonlinearity The ADC chip is specified to have a typical integral nonlinearity of 2 least-significant bits (LSB) or $0.003 \%$ of full range. Integral nonlin- 

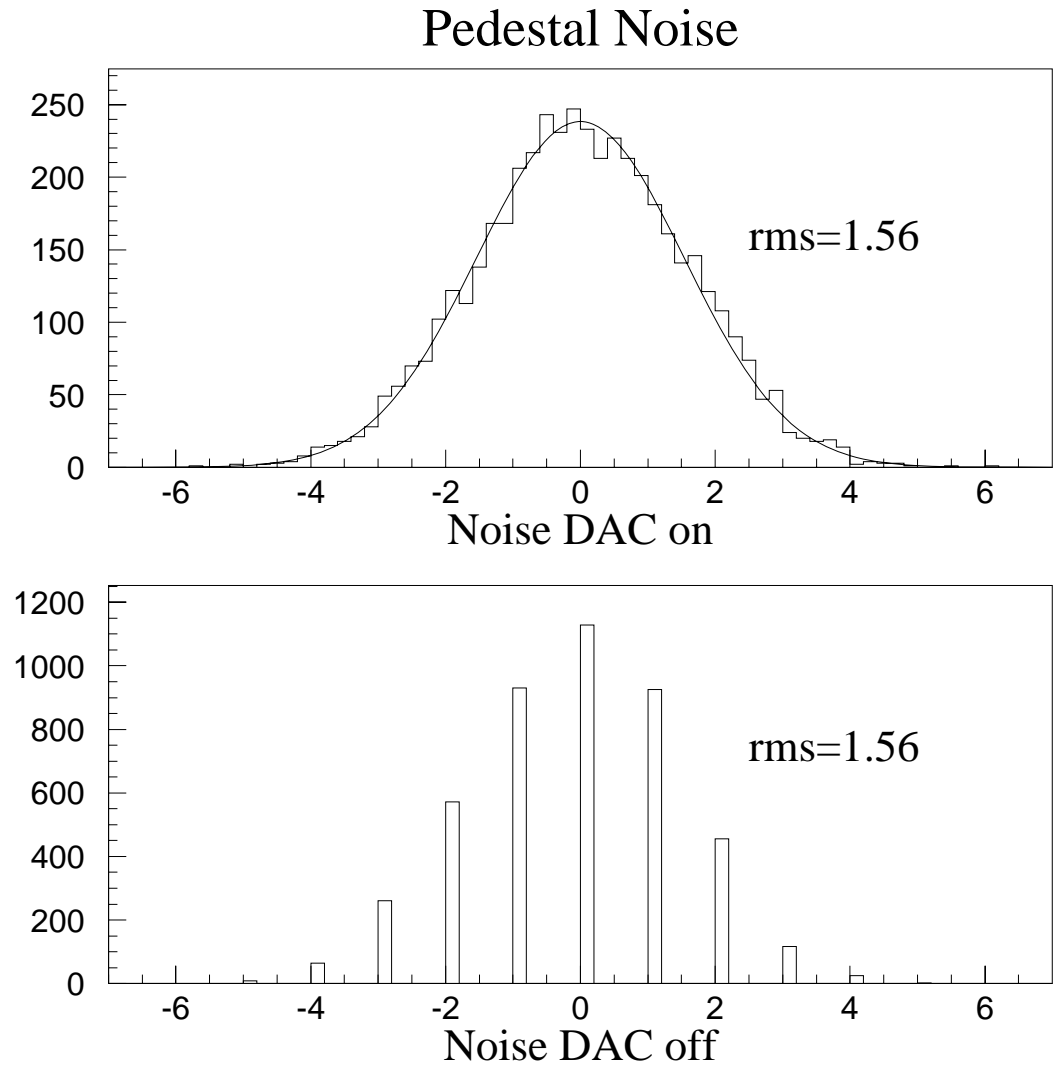

Figure A.10: A histogram of the pedestal noise of a typical ADC channel. DAC noise is used in the top plot. Pedestal noise is 1.56 ADC bins in both cases.

earity is defined to be the maximum deviation from a straight line connecting the endpoints of the ADC transfer function. The integral nonlinearity of the entire ADC channel has been measured to be at most 5-10 LSB. A plot of the integral nonlinearity of a typical ADC channel is shown in Figure A.11.

Differential Nonlinearity Although the differential nonlinearity of the AD7884 ADC chip is not specified, its guarantee of monotonicity implies that the differential nonlinearity must be less than $1 / 2 \mathrm{LSB}$ or $0.0008 \%$ of full range. The use of DAC 


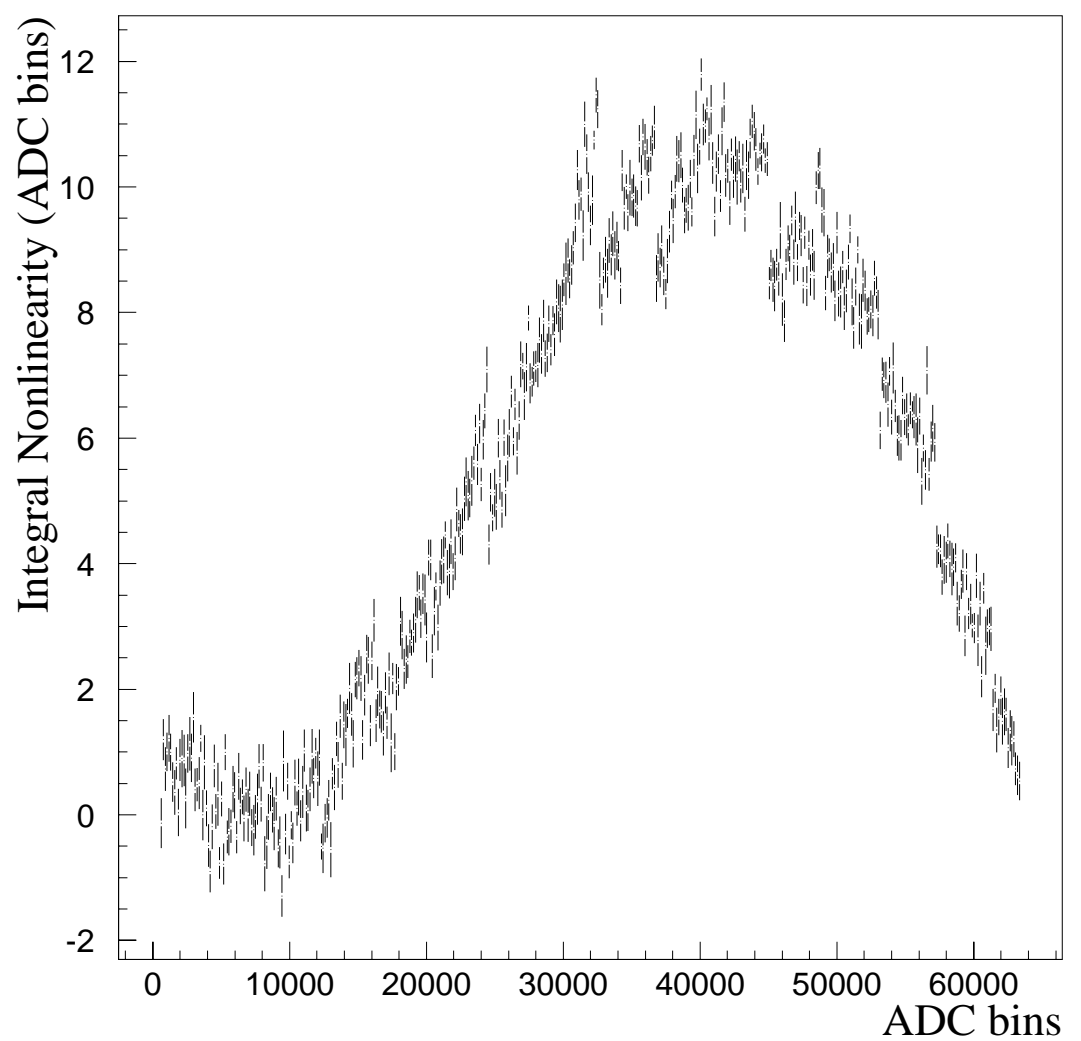

Figure A.11: Plot of the integral nonlinearity of an ADC channel. The input signal is provided by an AD669 16-bit DAC. Much of the nonlinearity shown is due to the measurement DAC. The plot does, however, establish an upper bound for the integral nonlinearity of the ADC of about 10 LSB or $0.015 \%$.

noise should reduce this value even further. The differential nonlinearity has been measured to be much less than 1 LSB with and without DAC noise.

Performance Limitations The ADC board is designed to integrate relatively slow signals for repetitive fixed intervals. Consequently its performance deteriorates for signals faster than $1 \mathrm{MHz}$ or for consecutive integrated results which differ by more than $50 \%$ of full scale. Signals faster than $1 \mathrm{MHz}$ may be accommodated by 
changing the op-amp in the differential amplifier from the AD795 to one with a faster slew rate. Pedestal noise may increase, however.

\section{Instructions for Using the ADC Boards}

Connections The ADC board is inserted into a $9 \mathrm{U}$ slot in the VME crate. The crate should be powered down before inserting or removing the board. A diagram of the front panel connections is shown in Figure A.12. There are three types of connectors on the front panel: the analog inputs, timing signal input connector, and diagnostic outputs, each described below.

The analog input for each ADC channel is a BNC-style twin-axial connector. The outer shield is at ground, and the two inner pins carry the input signal. For voltage-integrating channels, the female pin is the positive input to the differential amplifier input stage. For current-integrating channels, the current should flow into the female pin. Since signal sources typically do not have twin-axial outputs, the signal must be converted to twin-ax somewhere between the source and the ADC input.

The timing signals are input through a 10-pin connector. The signals are differential positive ECL (PECL). The order and polarity of the timing signals are given below.

$$
\begin{array}{|c|l|}
\hline-+ & \text { unused } \\
-+ & \text { Reset } \\
-+ & \text { Convst } \\
-+ & \text { Peak } \\
-+ & \text { Baseline } \\
\hline
\end{array}
$$

Two diagnostic output signals are provided. The signal labeled INT OUT is an analog copy of the output of the integrator. This signal typically has a sawtooth shape and is the voltage ramp referred to in Section A.3.1. The signal labeled SUM 


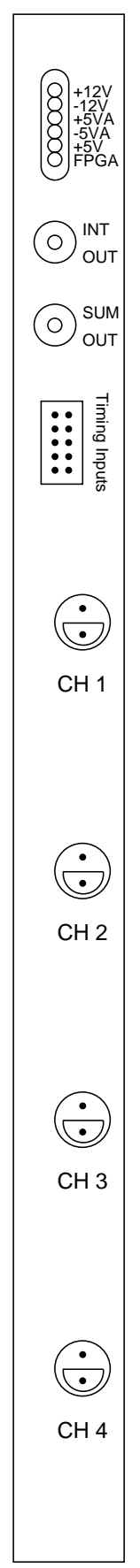

Figure A.12: ADC front panel connections. 
OUT is an analog copy of the output of the summing amplifier. This is the final signal which is digitized by the ADC chip, after the DAC and offset have been added to the integrated result. The diagnostic outputs can be set to show any one of the four ADC channels. The particular ADC channel is selected by writing to the Diagnostic Mux, which is part of the Control Register at address $\$ C$.

Addressing Each ADC board occupies 256 bytes of 24-bit VME address space. The address of a register on the board is given in hexadecimal by 0xf0XXXXYY where $\$ \mathrm{XXXX}$ is the 16-bit base address and $\$ Y Y$ is the 8 -bit relative address.

The base address is set by a 16-bit jumper field labeled JMPR3. The most significant bit is in the top position (pins 1-2) and an inserted jumper corresponds to a zero. Each ADC board must be assigned a unique base address.

The address map for the ADC board is given in Table A.1. Note that this table is different from the one given in reference [Oliver], which is in error. The addresses are given relative to the base address. For example, the Control Register for a board with base address $\$ 1234$ would be located at address 0xf012340c.

Some ADC boards have had problems recognizing their own addresses, causing VMEbus errors when attempting to read from or write to addresses on these boards. These addressing problems were investigated and found to be consistent with cold solder joints. The defective boards have been repaired by touching up the relevant solder joints. Since the nature of this problem is intermittent and unpredictable, it is possible that previously unaffected boards could display addressing problems in the future. If a board does not address properly, all solder joints should be touched up and then the board should be tested to see if that cures the problem. (U26-29, for starters.) 


\begin{tabular}{|c|c|c|c|c|}
\hline $\begin{array}{r}\text { Hex } \\
\text { Address }\end{array}$ & $\begin{array}{l}\text { Register } \\
\text { Name }\end{array}$ & $\begin{array}{l}\text { Read/ } \\
\text { Write }\end{array}$ & Bit & Function \\
\hline$\$ 0$ & ADC channel 1 & $\mathrm{R}$ & & 16-bit ADC data \\
\hline$\$ 2$ & ADC channel 3 & $\mathrm{R}$ & & 16-bit ADC data \\
\hline$\$ 4$ & ADC channel 2 & $\mathrm{R}$ & & 16-bit ADC data \\
\hline$\$ 6$ & ADC channel 4 & $\mathrm{R}$ & & 16-bit ADC data \\
\hline$\$ 8$ & Noise DAC & W & & 16-bit DAC value \\
\hline$\$ A$ & unused & & & \\
\hline \multirow[t]{5}{*}{$\$ C$} & Control Register & $\mathrm{R} / \mathrm{W}$ & Bit $0-1$ & Diagnostic Mux Address \\
\hline & & & Bit 2 & Enable interrupts \\
\hline & & & Bit 3 & Enable RNG \\
\hline & & & Bit 4 & Integrator range select \\
\hline & & & Bit 5-7 & unused \\
\hline$\$ E$ & unused & & & \\
\hline$\$ 10$ & $\begin{array}{l}\text { Random Number } \\
\text { Generator }\end{array}$ & & & \\
\hline$\$ 12$ & Status Register & $\mathrm{R}$ & Bit 0 & !DONE (Digitization complete) \\
\hline & & & Bit 1-7 & unused \\
\hline$\$ 14-\$ F E$ & unused & & & \\
\hline
\end{tabular}

Table A.1: Address map for ADC board. All addresses are relative to the base address.

Readout The 16-bit digitized values for each ADC channel appear at relative addresses $\$ 00-\$ 06$, as shown in Table A.1. The digitized values are given in twoscomplement format. Notice that successive channel addresses correspond to the front panel inputs in non-ascending order. Thus the addresses for channels 2 and 3 appear to be swapped. This order carries over to the Diagnostic Mux, so that its addressing is given by:

$\begin{array}{cc}\text { Mux[1:0] } & \text { ADC channel } \\ 00 & 1 \\ 01 & 3 \\ 10 & 2 \\ 11 & 4\end{array}$

The board is supposed to be capable of generating a VMEbus interrupt when it has valid ADC data, but this feature has never been used (or tested) for HAPPEX. 
It can also be operated in polling mode, in which the Status Register at address $\$ 12$ is continuously polled until the DONE bit is observed to be asserted, indicating that the analog-to-digital conversion is complete. We have not operated in polling mode either. Instead, the ADC Timing Board, described in Section A.3.2 sends the VME Trigger signal to the VME Trigger Interface module $7.5 \mu$ s after the Convst signal has been strobed. (It takes $5.3 \mu$ s for the ADC chip to complete digitization. The VME Trigger Interface module then triggers CODA and causes the ADC boards to be read out.

The VME computer can access ADC addresses across the VME backplane. The following segment of $\mathrm{C}$ code sets the $\mathrm{DAC}$ to $\$ 9 \mathrm{~A} 3 \mathrm{E}$ and reads out channel 1 on the ADC board with base address $\$ 1234$.

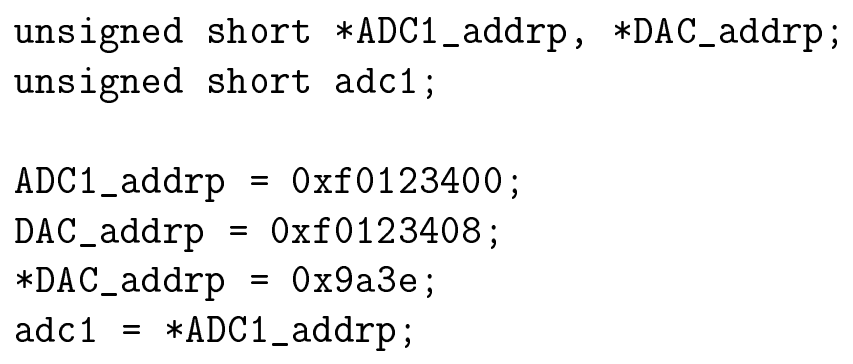

DAC Noise The 16-bit DAC value may be supplied by a random number generator (RNG) on the ADC board or by the VME computer. Unfortunately, the DAC exhibits a severe nonlinearity at either end of its range, as shown in Figure A.13. For this reason the RNG, which has no method of range selection, should not be used. Instead a random DAC value between 2048 and 63488 ( $\$ 0800$ and $\$ F 800$ ) must be generated by the VME computer and written to the DAC immediately after reading out the ADC data.

Figure A.13 shows the ADC response to the DAC for zero input to the ADC channel. The response over the linear range must be calibrated in order to correctly 

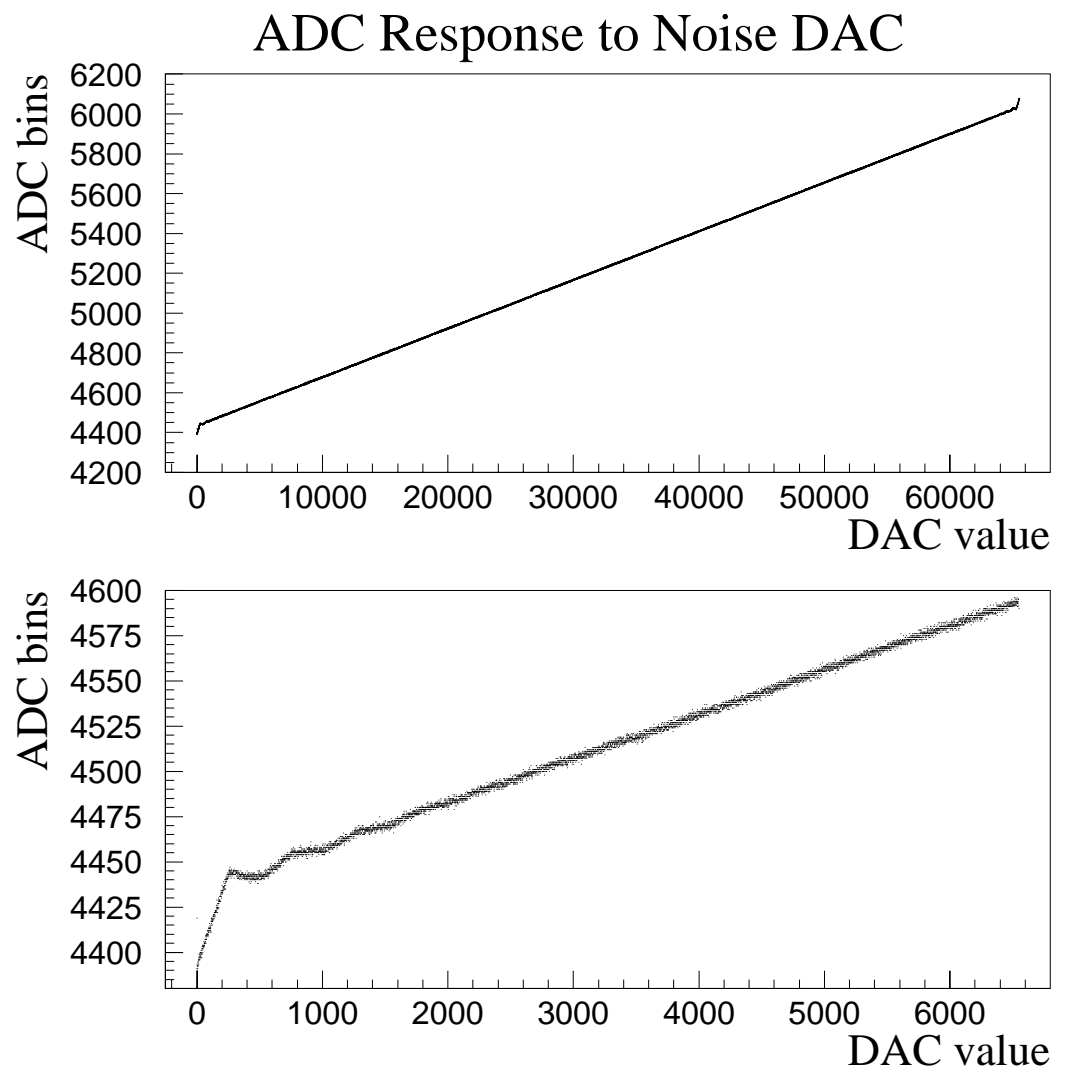

Figure A.13: Plots of the ADC response to the noise DAC. The top plot shows the nonlinearity at either end of the DAC range. The bottom plot zooms in on the lower end of the DAC range. The response is linear for DAC values greater than $\sim 2000$ and less than $\sim 63500$.

subtract the DAC contribution from the integrated ADC result. Each ADC channel should be calibrated separately, since component variation causes small differences in the ADC response. Calibration is performed by fitting a straight line to the linear response curve and noting its slope $m$ and intercept $b$. Then the corrected ADC data can be recovered using the formula

$$
\mathrm{ADC}_{\text {corrected }}=\mathrm{ADC}-m \cdot \mathrm{DAC}-b .
$$

Typical values are $m=0.002445$ and $b=4400$. The intercept for a given ADC 


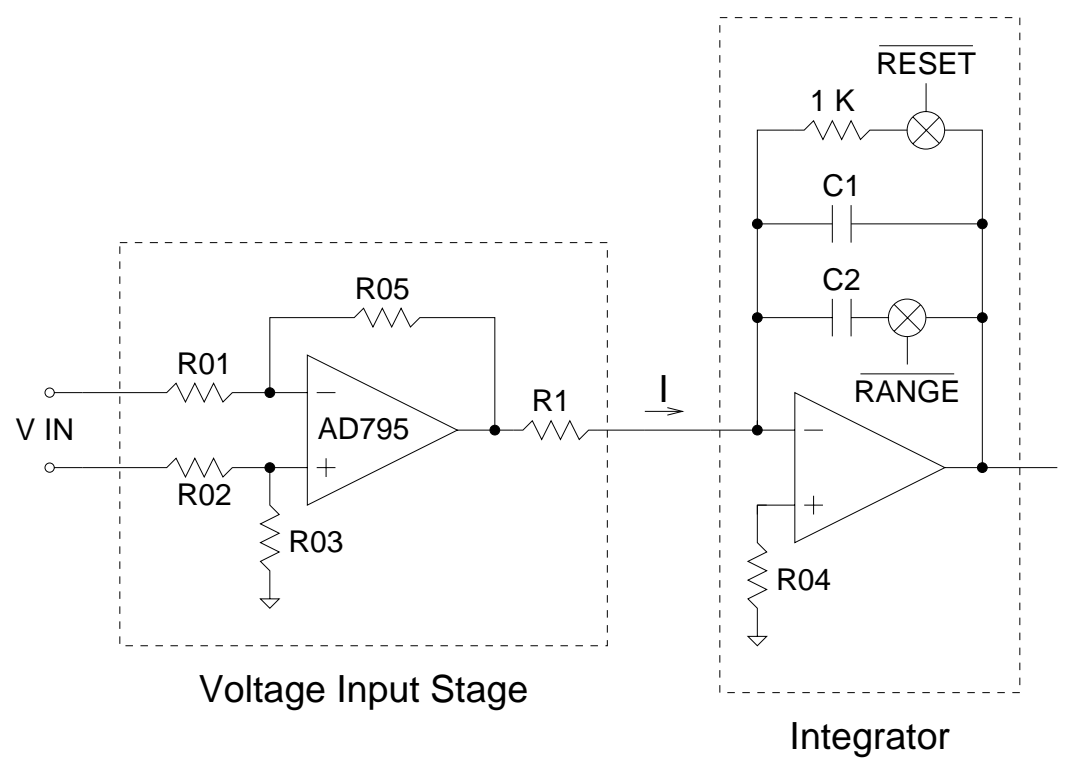

Figure A.14: Input stage and integrator for voltage-integrating ADC channel. Resistor R04 should be $2 \mathrm{~K}$. The other resistors and capacitors can be adjusted to change the gain of the channel.

channel is observed to drift by a few tens of ADC bins, but the slope remains essentially constant over time.

Changing the Integrator Gain The integrator gain should be optimized to use the full dynamic range of the ADC channel. Two integrating capacitors are provided to allow easy switching between two different integrator gains. Integrating capacitor $\mathrm{C} 1$ is used for high gain and $\mathrm{C} 1+\mathrm{C} 2$ is used for low gain. High or low gain is selected by writing to bit 4 of the Control Register.

On-board modifications are necessary in order to change the existing gain range of the integrator or to convert a channel from voltage-integrating to current-integrating. Voltage-integrating and current-integrating channels differ only in the input stage and integrator.

Figure A.14 shows a voltage-integrating channel. The gain $G_{V}$ of a voltageintegrating ADC channel is determined by resistors R01, R02, R03, R05, and R1; 
by capacitors $\mathrm{C} 1$ and $\mathrm{C} 2$; and by the integration time $\Delta t$ :

$$
G_{V}=\frac{\mathrm{R} 05 \cdot \Delta t}{\mathrm{C} \cdot \mathrm{R} 1 \cdot \mathrm{R} 01},
$$

where $\mathrm{C}=\mathrm{C} 1$ if the low gain range is selected and $\mathrm{C}=\mathrm{C} 1+\mathrm{C} 2$ if the high gain range is selected.

Resistor R04 should be $2 \mathrm{k} \Omega$. Resistors R01, R02, R03, and R05 must be chosen so that

$$
\frac{\mathrm{R} 01}{\mathrm{R} 05}=\frac{\mathrm{R} 02}{\mathrm{R} 03}
$$

Ideally, $\mathrm{R} 01=\mathrm{R} 02+\mathrm{R} 03$ should also hold so that both input wires see the same impedance to ground. ${ }^{3}$ This maximizes common-mode noise rejection. However, it is probably good enough to use $\mathrm{R} 01=\mathrm{R} 02$ and $\mathrm{R} 05=\mathrm{R} 03$. Precision resistors with $0.1 \%$ tolerance are used in order to ensure precise impedance matching, although in a pinch $1 \%$ resistors may be used. Resistors should stay in the range $1 \mathrm{k} \Omega$ to $100 \mathrm{k} \Omega$ in order to keep currents reasonable. Capacitors should be in the range $100 \mathrm{pf}$ to $.04 \mu \mathrm{f}$.

Default resistor values for the input stage differential amplifier are $\mathrm{R} 01=11.00 \mathrm{k} \Omega$, $\mathrm{R} 05=1.100 \mathrm{k} \Omega, \mathrm{R} 02=10.00 \mathrm{k} \Omega$, and $\mathrm{R} 03=1.00 \mathrm{k} \Omega$. With these values, the differential amplifier in the input stage has a gain of one-tenth. Thus the differential amplifier actually attenuates the input signal. Gains greater than one are attainable with suitably selected resistor values, but care must be taken not to exceed the voltage rails of the op-amp. That is, the maximum input voltage signal times the gain (R05/R01) of the differential amplifier should not exceed 10 volts.

Figure A.15 shows a current-integrating channel. The gain $G_{C}$ of a currentintegrating channel is determined by the integrating capacitors $\mathrm{C} 1$ and $\mathrm{C} 2$ and by

\footnotetext{
${ }^{3}$ Private communication with John Oliver
} 


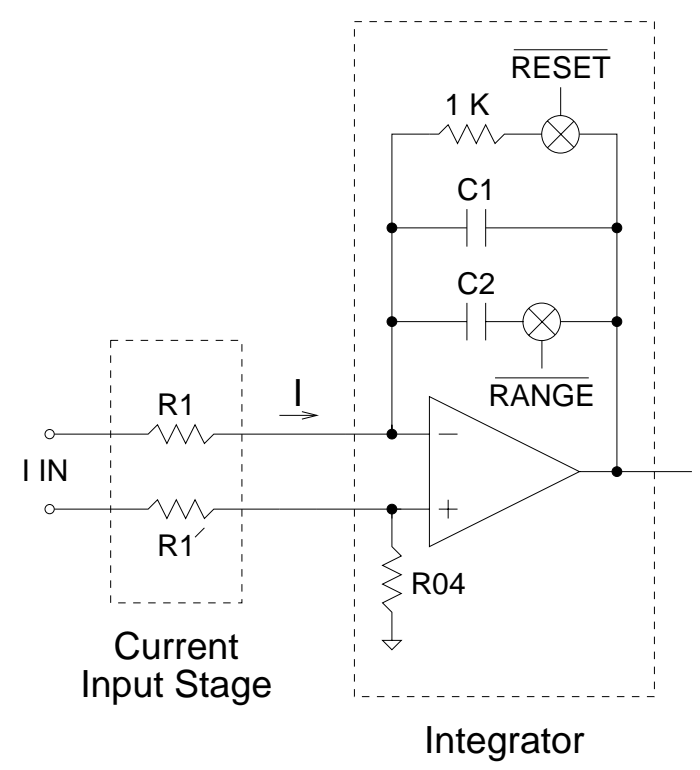

Figure A.15: Input stage and integrator for current-integrating ADC channel. Resistor R04 should be $50 \Omega$, and both R01 and R01' should be $1 \mathrm{k} \Omega$.

the integration time $\Delta t$ :

$$
G_{C}=\frac{\Delta t}{C}
$$

where $\mathrm{C}=\mathrm{C} 1$ if the low gain range is selected and $\mathrm{C}=\mathrm{C} 1+\mathrm{C} 2$ if the high gain range is selected.

Resistor R04 should be $50 \Omega$. Resistors R01 and R01' should both be $1 \mathrm{k} \Omega$. The input leads from the twin-ax bulkhead connect to different holes on the ADC board depending on whether the channel is current- or voltage-integrating. There is actually no place on the board reserved for resistor R01'. It must be inserted between the twin-ax bulkhead and the negative input lead.

\section{A.3.2 ADC Timing Board}

The ADC Timing Board generates the timing signals for the ADC boards, supplies the PITA Offset frequency to the HV Setpoint Generator as part of the intensity 
feedback loop, and processes the helicity signals from the Helicity Generator. Implementing this board as a VME module allows timing parameters and other information to be communicated to the VME computer across the VMEbus backplane. A circuit diagram of the ADC Timing Board is shown in Figure A.18. Much of its functionality, including implementation of the VMEbus protocol and generation of the timing signals, is embedded in a field-programmable gate array (FPGA).

\section{External Connections and Internal Addresses}

The ADC Timing board is inserted into a $6 \mathrm{U}$ slot in the VME crate. The crate should be powered down before inserting or removing the board. Figure A.16 shows a diagram of the front panel connectors. The ADC ribbon cable should be connected to the timing inputs on the ADC boards. Another ribbon cable connects to the VME Trigger Interface input port. The Master Trigger, Helicity, Realtime, and PITA Offset signals should be connected to the appropriate signals from the helicity control electronics. Diagnostic outputs are provided for the DAC voltages and the integrate gate.

The ADC Timing Board occupies 16 bytes of 16-bit VME address space. The address of a register on the board is given in hexadecimal by OxffffXXXY, where $\$ X X X$ is the 12-bit base address and $\$ Y$ is the relative address. The base address is set by a 12-bit switch on the ADC Timing Board. The address map in Table A.2 give the relative addresses of the registers on the ADC Timing Board. See Section A.3.1 for more information about writing to and reading from these addresses.

\section{ADC Timing}

Each ADC board requires external timing signals to perform the integration cycle. The ADC Timing Board supplies the required timing signals through the ADC 


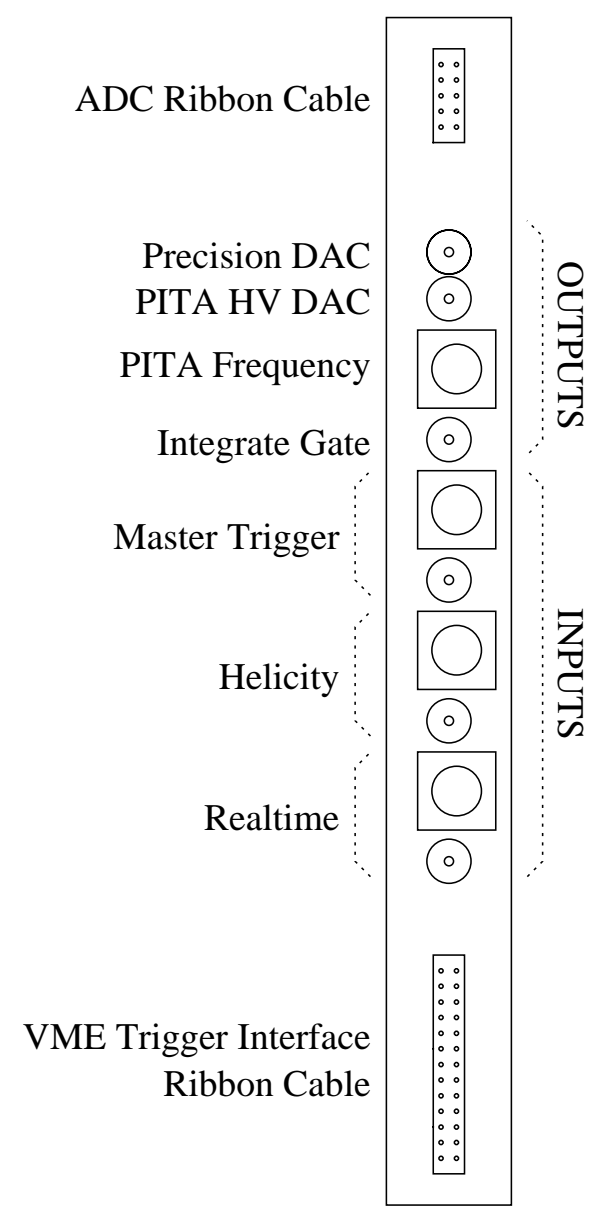

Figure A.16: Front panel of timing card.

ribbon cable.

All timing signals are based on a $20 \mathrm{MHz}$ crystal clock oscillator specified to have 50 ppm stability. This clock is divided down to $400 \mathrm{kHz}$ by counters buried in the FPGA to form the basic timing unit. All timing parameters are set as multiples of the $2.5 \mu$ s base time unit.

The ADC Timing Board generates five timing signals: Baseline, Peak, Reset, Convst, and VME Trigger. A timing diagram of the outputs is shown in Figure A.17. The timing cycle begins at the edge of the Master Trigger signal. The Ramp Delay is the delay between the edge of the Master Trigger and the beginning of the integrator 


\begin{tabular}{|r|l|c|l|l|}
\hline Hex & $\begin{array}{l}\text { Register } \\
\text { Address }\end{array}$ & $\begin{array}{c}\text { Read/ } \\
\text { Write }\end{array}$ & Bit & Description \\
\hline$\$ 0$ & Input Data & $\mathrm{R}$ & 0 & Data0 (Helicity) \\
& & $\mathrm{R}$ & 1 & Data1 (Realtime) \\
$\$ 2$ & unused & & & \\
$\$ 4$ & unused & & & 12-bit DAC value \\
$\$ 6$ & PITA DAC & $\mathrm{W}$ & & 16-bit DAC value \\
$\$ 8$ & Precision DAC & $\mathrm{W}$ & & \\
$\$ \mathrm{~A}$ & Ramp Delay & $\mathrm{R} / \mathrm{W}$ & & \\
$\$ \mathrm{C}$ & Integration Time & $\mathrm{R} / \mathrm{W}$ & & \\
$\$ \mathrm{E}$ & Oversample & $\mathrm{W}$ & $0-7$ & Oversample setting \\
& & $\mathrm{R}$ & $8-15$ & Current oversample \\
\hline
\end{tabular}

Table A.2: Address map for ADC Timing Board. All addresses are relative to the base address.

ramp. The Ramp Delay allows us to wait until the beam polarization has stabilized before beginning to integrate.

The Integration Time is the delay between the baseline and peak samples. The Ramp Delay and Integration Time are set as multiples of the $2.5 \mu$ s base time unit at addresses $\$ \mathrm{~A}$ and $\$ \mathrm{C}$, respectively. All other timing parameters shown in the timing diagram are fixed to optimize performance for HAPPEX. (See Section A.3.1 for details about the timing of the integration cycle.)

The Integrate Gate signal is provided as a diagnostic output on the front panel of the ADC Timing Board.

Oversampling The board has the ability to perform more than one timing cycle per helicity window. This feature is known as oversampling. Up to 256 cycles are possible for each master trigger. The number of oversamples is set at the lower 8 bits of address $\$$ E. Setting these bits to $\$ 00$ results in one timing cycle, while setting these bits to $\$ 01$ results in two timing cycles, etc. During the integration cycle, the oversample number of the current cycle can be read at the upper 8 bits of address 


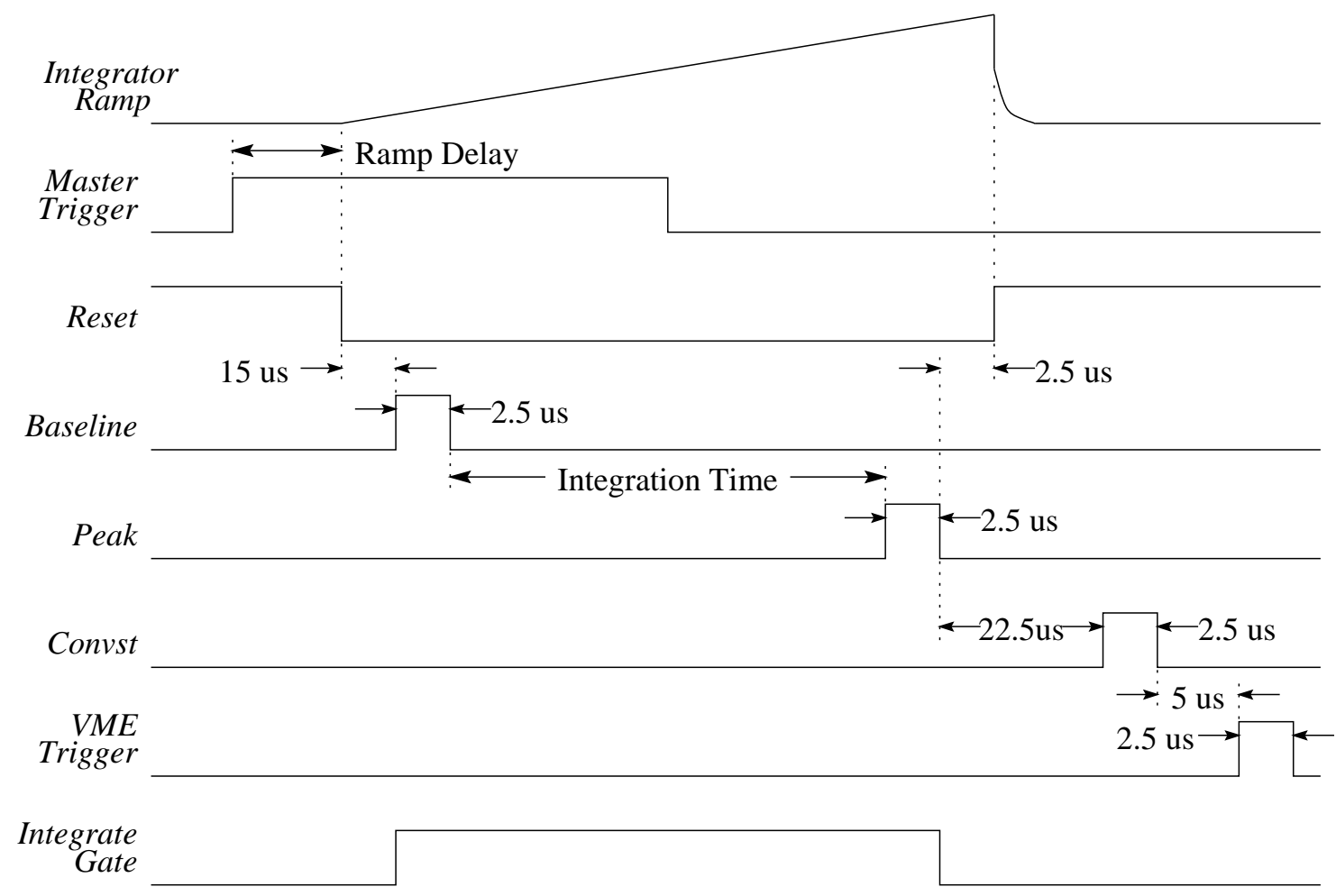

Figure A.17: Timing diagram for ADC Timing Board outputs.

\$E. The Ramp Delay occurs only before the first timing cycle, but not between oversamples.

\section{Input Signals}

The board accommodates three input signals: the Master Trigger signal and two data inputs. Both optical fiber and optically isolated TTL connectors are provided for each input signal.

The Master Trigger signal triggers the ADC timing cycle. Either the rising or falling edge of the Master Trigger may be selected as the trigger. The rising edge is selected by connecting pin 83 of the FPGA to $+5 \mathrm{~V}$, while the falling edge is selected by connecting pin 58 to $+5 \mathrm{~V}$. Both edges will trigger a timing cycle if both pins are tied high. A new timing cycle cannot be triggered while a cycle is in progress. 
Triggers which occur before the current cycle is completed are ignored.

The two data inputs are reserved for the Helicity and Realtime signals. These inputs are converted to ECL and sent out along with the VME Trigger signal to the VME Trigger Interface module. The data inputs can also be read across the VMEbus backplane at address $\$ 0$, bits 0 and 1 .

\section{DACs and V/F}

The board contains a 12-bit DAC and voltage-to-frequency converter ( $/ \mathrm{F}$ ) for generating the PITA frequency and a precision 16-bit DAC for ADC tests. The 12-bit DAC value is set at address $\$ 6$ and has an output range of 0 to 10 Volts. This voltage feeds the $\mathrm{V} / \mathrm{F}$, which has an output range of 0 to $100 \mathrm{kHz}$. The 16 -bit DAC is set at address $\$ 6$ and has an output range of -5 to +5 Volts. Both DAC voltages are provided as analog outputs and the $\mathrm{V} / \mathrm{F}$ output is available as an optical fiber signal. 


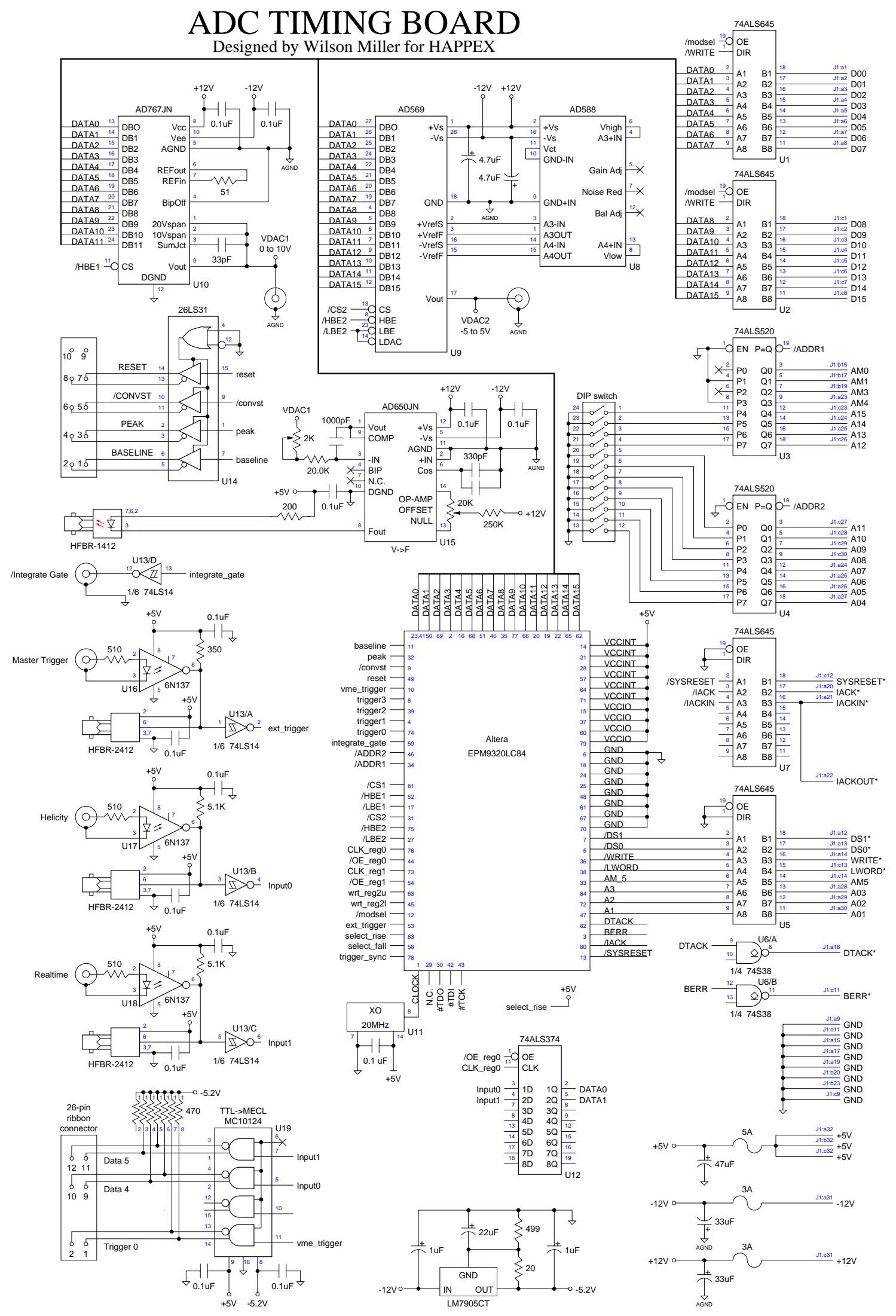

Figure A.18: Circuit diagram of ADC Timing Board. 


\section{Bibliography}

[1] David B. Kaplan and Aneesh Manohar. Strange matrix elements in the proton from neutral-current experiments. Nuclear Physics, B310:527, 1988.

[2] M. J. Musolf et. al. Intermediate-energy semileptonic probes of the hadronic neutral current. Physics Reports, 239, 1994.

[3] D. H. Beck. Strange-quark vector currents and parity-violating electron scattering from the nucleon and from nuclei. Physical Review D, 39:3248, 1989.

[4] C. Y. Prescott et. al. Further measurements of parity non-conservation in inelastic electron scattering. Physics Letters, 84B:524, 1979.

[5] W. Heil et. al. Improved limits on the weak, neutral, hadronic axial vector coupling constants from quasielastic scattering of polarized electrons. Nuclear Physics, B327:1, 1989.

[6] P. A. Souder et. al. Measurement of parity violation in the elastic scattering of polarized electrons from ${ }^{12}$ C. Physical Review Letters, 65:694, 1990.

[7] R. D. McKeown. Sensitivity of polarized elastic electron-proton scattering to the anomalous baryon number magnetic moment. Physics Letters B, 219:140, 1989.

[8] M. N. Rosenbluth. High energy elastic scattering of electrons on protons. Physical Review, 79:615, 1950.

[9] S. Galster et. al. Elastic electron-deuteron scattering and the electric neutron form factor at four-momentum transfers $5 \mathrm{fm}^{-2}<q^{2}<14 \mathrm{fm}^{-2}$. Nuclear Physics, B32:221, 1971.

[10] B. Mueller et. al. Measurement of the proton's neutral weak magnetic form factor. Physical Review Letters, 78:3824, 1997.

[11] D. T. Spayde et. al. Parity violation in elastic electron-proton scattering and the proton's strange magnetic form factor. Physical Review Letters, 84:1106, 2000 . 
[12] Gordon D. Cates. PhD thesis, Yale University, 1987.

[13] Krishna S. Kumar. Parity Violation in Elastic Electron Carbon Scattering. PhD thesis, Syracuse University, January 1990.

[14] M. Poelker. High power gain-switched diode laser master oscillator and amplifier. Applied Physics Letters, 67:2762, 1995.

[15] Matt Poelker and John Hansknecht. A high power gain switched diode laser oscillator and amplifier for the CEBAF polarized electron injector. Technical Report JLAB-ACC-96-19, Jefferson Laboratory, 1996.

[16] Matt Poelker and John Hansknecht. A diode laser system for synchronous photoinjection. Technical Report JLAB-ACC-97-26, Jefferson Laboratory, 1997.

[17] W. Barry, J. Heefner, and J. Perry. Electronic systems for beam position monitors at CEBAF. Technical Report CEBAF-PR-90-023, Continuous Electron Beam Accelerator Facility, October 1990.

[18] Walter Barry. A general analysis of thin wire pickups for high frequency beam position monitors. Technical Report CEBAF-PR-90-024, Continuous Electron Beam Accelerator Facility, October 1990.

[19] Riad Suleiman. Hall A cryogenic and dummy targets information. Technical Report TN-98-007, Thomas Jefferson National Accelerator Facility, 1998.

[20] William E. Kahl. A Measurement of the Strange Quark Content of the Proton. PhD thesis, Syracuse University, 2000.

[21] C. Yan et. al. Beam raster system at CEBAF. Technical Report CEBAF-PR93-005, Continuous Electron Beam Accelerator Facility, 1993.

[22] John Oliver and Krishna Kumar. CEBAF-91-010 Integrating ADC.

[23] P. J. E. Peebles. Quantum Mechanics. Princeton University Press, 1992.

[24] Noah Sherman. Physical Review, 103:1501, 1956.

[25] Charles K. Sinclair. Electron beam polarimetry. Technical Report JLAB-ACC98-04, Jefferson Laboratory, 1998.

[26] J. S. Price et. al. $5 \mathrm{MeV}$ Mott polarimeter for rapid precise electron beam polarization measurements. Technical Report JLAB-ACC-96-22, Jefferson Laboratory, 1996.

[27] J. S. Price et. al. $5 \mathrm{MeV}$ Mott polarimeter development at Jefferson Lab. Technical Report JLAB-ACC-97-27, Jefferson Laboratory, 1997. 
[28] V. B. Berestetskii, E. M. Lifshitz, and L. P. Pitaevskii. Quantum Electrodynamics, volume 4 of Landau and Lifshitz Course of Theoretical Physics. Pergamon Press, Oxford, 1982.

[29] A. V. Glamazdin et. al. Electron beam Møller polarimeter at JLab Hall A. Fizika B, 8:91-95, 1999.

[30] Peter A. Mastromarino. Beam Fluctuations in Parity-Violating Electron Scattering Experiments. A. B. Thesis, Princeton University, April 1999.

[31] K. A. Aniol et. al. Measurement of the neutral weak form factors of the proton. Physical Review Letters, 82:1096, 1999.

[32] R. L. Jaffe. Stranger than fiction: The strangeness radius and magnetic moment of the nucleon. Physics Letters B, 229:275, 1989.

[33] H.-W. Hammer, Ulf-G. Meissner, and D. Drechsel. The strangeness radius and magnetic moment of the nucleon revisited. Physics Letters B, 367:323, 1996.

[34] M. J. Musolf and M. Burkardt. Stranger still: kaon loops and strange quark matrix elements of the nucleon. Zeitschrift für Physik C, 55:3066, 1997.

[35] H. Weigel et. al. On the strange vector form factors of the nucleon in the NJL soliton model. Physics Letters B, 353:20, 1995.

[36] N. W. Park, J. Schechter, and H. Weigel. Electromagnetic, axial-vector, and strange currents in the Skyrme model: Effects of symmetry breaking. Physical Review D, 43:869, 1991.

[37] S. J. Dong, K. F. Liu, and A. G. Williams. Lattice calculation of the strangeness magnetic moment of the nucleon. Physical Review D, 58:074504, 1998.

[38] P. Geiger and N. Isgur. Strange hadronic loops of the proton: A quark model calculation. Physical Review D, 55:299, 1997. 